\title{
Simulating the Growth of a Disk Galaxy and its Supermassive Black Hole in a Cosmological Context \\ by
}

\section{Robyn Levine}

B.A., University of Pittsburgh, 2001

B.S., University of Pittsburgh, 2001

M.S., University of Colorado, 2005

A thesis submitted to the

Faculty of the Graduate School of the

University of Colorado in partial fulfillment

of the requirements for the degree of

Doctor of Philosophy

Department of Astrophysical \& Planetary Sciences

2008 
This thesis entitled:

Simulating the Growth of a Disk Galaxy and its Supermassive Black Hole in a Cosmological Context written by Robyn Levine has been approved for the Department of Astrophysical \& Planetary Sciences

Prof. Andrew J. S. Hamilton

Prof. Phillip J. Armitage

Prof. Mitchell C. Begleman

Prof. Peter L. Bender

Prof. Nickolay Y. Gnedin

Date

The final copy of this thesis has been examined by the signatories, and we find that both the content and the form meet acceptable presentation standards of scholarly work in the above mentioned discipline. 
Levine, Robyn (Ph.D., Astrophysics)

Simulating the Growth of a Disk Galaxy and its Supermassive Black Hole in a Cosmological Context

Thesis directed by Prof. Andrew J. S. Hamilton

Supermassive black holes (SMBHs) are ubiquitous in the centers of galaxies. Their formation and subsequent evolution is inextricably linked to that of their host galaxies, and the study of galaxy formation is incomplete without the inclusion of SMBHs. The present work seeks to understand the growth and evolution of SMBHs through their interaction with the host galaxy and its environment. In the first part of the thesis (Chap. 2 and 3), we combine a simple semi-analytic model of outflows from active galactic nuclei (AGN) with a simulated dark matter density distribution to study the impact of SMBH feedback on cosmological scales. We find that constraints can be placed on the kinetic efficiency of such feedback using observations of the filling fraction of the Ly $\alpha$ forest. We also find that AGN feedback is energetic enough to redistribute baryons over cosmological distances, having potentially significant effects on the interpretation of cosmological data which are sensitive to the total matter density distribution (e.g. weak lensing). However, truly assessing the impact of AGN feedback in the universe necessitates large-dynamic range simulations with extensive treatment of baryonic physics to first model the fueling of SMBHs. In the second part of the thesis (Chap. 4-6) we use a hydrodynamic adaptive mesh refinement simulation to follow the growth and evolution of a typical disk galaxy hosting a SMBH, in a cosmological context. The simulation covers a dynamical range of 10 million allowing us to study the transport of matter and angular momentum from super-galactic scales all the way down to the outer edge of the accretion disk around the SMBH. Focusing our 
attention on the central few hundred parsecs of the galaxy, we find the presence of a cold, self-gravitating, molecular gas disk which is globally unstable. The global instabilities drive super-sonic turbulence, which maintains local stability and allows gas to fuel a SMBH without first fragmenting completely into stars. The fueling appears to be a stochastic process, with no preferred timescale for accretion over the duration of the simulation. 


\section{Dedication}

To my grandparents for being proud of me anyway. 


\section{Acknowledgements}

There are many people I wish to acknowledge who have made some contribution to the successful completion of this thesis, whether directly, or through a supportive role. Unfortunately, not everyone who deserves it can fit on this page. So here are just a few...

None of the work presented in this thesis would have been possible without the much appreciated advising and encouragement of Nick Gnedin and Andrew Hamilton. I have benefited greatly from both of their expertise, even and perhaps especially when they have disagreed with one another. Particularly, I thank Nick for the great trouble he has gone through to get me through graduate school in the midst of his own transition from life in Colorado to life in Chicago. And Andrew, I thank for his inspiring enthusiasm for all things black holes, which has helped me past any frustration I have encountered along the way.

I thank our collaborator throughout much of this work, Andrey Kravtsov at the University of Chicago for lending his scientfic expertise and his state-of-theart simulations to the project. Having access to the ART code made the entire project worthwhile!

I would like to acknowledge the other members of my thesis committee: Phil Armitage, Peter Bender, and Mitch Begelman, whose comments and suggestions have been enormously helpful at every stage of the research. Also, many thanks to Jo Ann Vandel in the APS department for answering the dozens of e-mails I have sent her while trying to figure out how to graduate from afar. 
My sincerest thanks to the Fermilab Center for Particle Astrophysics for hosting me during the last three years of my dissertation work. Their support has deeply enriched my scientific experience as a graduate student. Thanks especially to the members of the Oak Park carpool!

Lastly, I wish to acknowledge the love and support of my friends and family. Particularly Matt Muench for many years of unparalleled friendship, my two sisters Lori and Jennie who I have always and will always look up to (both literally and figuratively), my parents for making me the person I am (again, both literally and figuratively), and Matt Browning for general awesomeness and about a million other things that won't fit within the page limit. 


\section{Contents}

\section{Chapter}

1 INTRODUCTION 1

1.1 Supermassive Black Holes . . . . . . . . . . . . . . . . . . . . 1

1.2 Feedback from Active Galactic Nuclei . . . . . . . . . . . . . . . 2

1.3 Coevolution of SMBHs and Their Hosts . . . . . . . . . . . . . 5

1.4 Numerical Progress . . . . . . . . . . . . . . . . . . . . . . . . . . 7

2 AGN OUTFLOWS IN A COSMOLOGICAL CONTEXT 10

2.1 Simulating the AGN Environment and Distribution . . . . . . . . 10

2.1.1 Cosmological Density Distribution . . . . . . . . . . 11

2.1.2 Luminosity Function . . . . . . . . . . . . . . . 12

2.2 AGN Outflows ..................... 15

2.2.1 Distribution of Outflows . . . . . . . . . . . . 15

2.2.2 Evolution of Outflows . . . . . . . . . . . . . . 17

2.2.3 Kinetic Luminosity and Filling Fraction . . . . . . . . . . 21

2.3 Comparison with a Poisson Distribution . . . . . . . . . . 23

2.4 Results ........................ . . . 24

2.4.1 Convergence Studies . . . . . . . . . . . . . . . . 24

2.4 .2 AGN Lifetime . . . . . . . . . . . . . . . 28

2.4.3 AGN Bias .................. . . . . 30

2.5 Discussion and Conclusions . . . . . . . . . . . . . . . 32 
3 AGN OUTFLOWS AND THE MATTER POWER SPECTRUM 35

3.1 Simulation . . . . . . . . . . . . . . . . . 35

3.1.1 A Simple Outflow Model . . . . . . . . . . . . . . . 35

3.1.2 Determining the Matter Power Spectrum . . . . . . . . . 36

3.2 Results . . . . . . . . . . . . . . . . . . . . 39

3.2.1 Convergence Studies . . . . . . . . . . . . . . . . . . . 39

3.2.2 Dependence of the Matter Power Spectrum on Kinetic Frac-



3.3 Discussion and Conclusions . . . . . . . . . . . . . . . 43

4 FROM MEGAPARSECS TO MILLIPARSECS: A COSMOLOGICAL SIMULATION MODELING BLACK HOLE GROWTH 45

4.1 Introduction and Motivation for doing the simulations . . . . . . 45

$4.2 \mathrm{ART} \ldots \ldots \ldots \ldots \ldots \ldots$

4.3 "Zooming In" to the Center of a Galaxy . . . . . . . . . . . . . 47

4.4 Numerical Issues . . . . . . . . . . . . . . . . . . . . . . . . 53

4.4.1 A Test of Angular Momentum Conservation . . . . . . . . 53

4.4.2 Dark Matter Discreteness Effects . . . . . . . . . . 56

4.4.3 Stability Against Artificial Fragmentation . . . . . . . . . 58

5 RESOLVING GAS DYNAMICS IN THE CIRCUMNUCLEAR REGION $\begin{array}{ll}\text { OF A SIMULATED DISK GALAXY } & 60\end{array}$

5.1 Introduction . . . . . . . . . . . . . . . . 60

5.2 Matter Transport in the Circumnuclear Region . . . . . . . . . . . 61

5.2.1 Structure of the Circumnuclear Disk . . . . . . . . . . 61

5.2.2 Angular Momentum Transport . . . . . . . . . . 66

5.2.3 Disk Stability and the Source of Turbulence . . . . . . . . 70

5.2.4 The Nature of the Turbulence . . . . . . . . . . . . 75 
5.3 Measuring the Viscosity . . . . . . . . . . . . . . . 81

5.4 Angular Momentum Conservation . . . . . . . . . . . . . . . . 82

5.5 Possible Effects of Missing Physics . . . . . . . . . . . . . . . . . 84

5.5.1 Optically Thick Cooling . . . . . . . . . . . 85

5.5.2 Magnetic Fields . . . . . . . . . . . . . . 87

5.6 Discussion and Conclusions _. . . . . . . . . . . . . . . . 89

6 MATTER TRANSPORT IN THE HOST GALAXY OF A SUPERMAS$\begin{array}{ll}\text { SIVE BLACK HOLE } & 92\end{array}$

6.1 SIMULATION . . . . . . . . . . . . . . . . . . . . 92

6.2 RESULTS . . . . . . . . . . . . . . . . . . . . . 94

6.2.1 Mass of the Disk and the Rate of Transport . . . . . . . . 95

6.2.2 Angular Momentum . . . . . . . . . . . . . 103

6.3 DISCUSSION AND CONCLUSIONS . . . . . . . . . . . 106

7 CONCLUSIONS AND THE DIRECTION OF FUTURE RESEARCH 108

7.1 Summary of Results . . . . . . . . . . . . . . . . . . 108

7.2 Future Work with the ART Code . . . . . . . . . . . . . . . 110

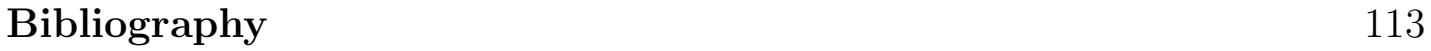




\section{Tables}

\section{Table}

2.1 Parameterization of the luminosity function . . . . . . . . . . 13

4.1 Initial conditions for collapsing sphere test . . . . . . . . . . . . 55

6.1 Summary of each simulation run . . . . . . . . . . . . . . . 94 


\section{Figures}

\section{Figure}

2.1 Comoving number density of AGN as a function of redshift . . . . 14

2.2 Comoving radii of AGN bubbles in different environments . . . . 20

2.3 Outflow filling fraction for different kinetic fractions . . . . . . . . 22

2.4 Outflow filling fraction for uniform and evolving density distributions 25

2.5 Outflow filling fraction for different box sizes . . . . . . . . . . 26

2.6 Cross sections of the density distribution for different resolution simulations . . . . . . . . . . . . . . . . . . 27

2.7 Outflow filling fractions for different resolutions . . . . . . . . 28

2.8 Outflow filling fractions for different AGN lifetimes . . . . . . . . 30

2.9 Outflow filling fractions for different AGN lifetimes (uniform density distribution) . . . . . . . . . . . . . . . . 31

2.10 Outflow filling fractions for different bias parameters . . . . . . 33

3.1 Change in matter power spectrum for different resolution simulations 40

3.2 Change in matter power spectrum for different size boxes . . . . . 41

3.3 Change in matter power spectrum over redshift . . . . . . . . . 42

3.4 Change in matter power spectrum with different kinetic fractions 43

4.1 Stellar component of simulated disk galaxy . . . . . . . . . . . . . 48

4.2 Interior gas mass during zoom-in part of simulation . . . . . . . . 50 
4.3 Contribution of individual cells to black hole particle mass . . . . 52

4.4 Volume rendering of gas density at different scales . . . . . . . . 54

4.5 Angular momentum distribution of collapsing sphere . . . . . . . 56

4.6 Velocity vs. radius of a sample of dark matter particles . . . . . . 58

5.1 Eigenvalues of the inertia tensor in the circumnuclear disk . . . . 62

5.2 Radial and tangential velocity components of the disk . . . . . . . 64

5.3 Gas density profile of the circumnuclear disk . . . . . . . . . . . 65

5.4 Comparison of tangential velocity, turbulent velocity, and sound speed .......................... 66

5.5 Comparison of torques transporting angular momentum . . . . . . 69

5.6 Evolution of disk instabilities . . . . . . . . . . . . . . . 71

5.7 Toomre $Q$-parameter and fastest growing unstable modes . . . . . 74

5.8 Ratio of local Jeans length to cellsize; distribution of level 19 and 20 cells . . . . . . . . . . . . . . . 76

5.9 Turbulence velocity vs. scale; comparison of scale with fastest growing unstable mode . . . . . . . . . . . . . . . . . . . . 78

5.10 Individual components of the velocity dispersion . . . . . . . . 79

5.11 Gas density PDF . . . . . . . . . . . . . . . . 80

5.12 Mass-weighted profiles of passive scalar . . . . . . . . . . . . . 83

5.13 Kinematic viscosity measured from diffusing scalar . . . . . . . . 84

5.14 Angular momentum as a function of interior gas mass . . . . . . . 85

5.15 Column density of gas . . . . . . . . . . . . 86

5.16 Minimum magnetic field necessary to influence gas dynamics . . . 88

6.1 Average interior gas mass for different simulations . . . . . . . . 96

6.2 Fluctuations in the gas mass in different redshift simulations . . . 97

6.3 Fourier transform of mass accretion rate (different redshifts) . . . 99 
6.4 Fourier transform of accretion rate (different black hole masses) . 100

6.5 Mean gas mass from each simulation as a function of cosmological

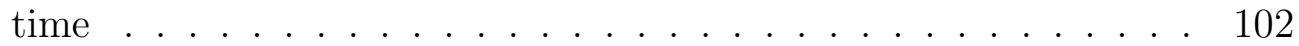

6.6 Map of angular momentum vectors at 100 and $1 \mathrm{pc}$. . . . . . . 105 


\section{Chapter 1}

\section{INTRODUCTION}

\subsection{Supermassive Black Holes}

Observations suggest that most galaxies host massive compact objects in their centers, likely to be supermassive black holes (SMBHs) with masses ranging from $10^{6}$ solar masses $\left(M_{\odot}\right)$ to more than $\sim 10^{9} M_{\odot}$ (e.g. Kormendy \& Richstone, 1995; Magorrian et al., 1998; Kormendy \& Gebhardt, 2001). Such massive objects likely grow from smaller mass "seeds" whose origins are, as of yet, uncertain. Some formation scenarios for the seeds of SMBHs include (1) the direct collapse of lowangular momentum gas in protogalactic halos (Haehnelt \& Rees, 1993; Umemura et al., 1993; Loeb \& Rasio, 1994; Eisenstein \& Loeb, 1995; Gnedin, 2001; Bromm \& Loeb, 2003; Koushiappas et al., 2004; Spaans \& Silk, 2006), or of the cores of quasi-stellar objects (Begelman et al., 2006); (2) the collapse of massive, early Population III stars (Carr et al., 1984; Larson, 2000; Schneider et al., 2000; Madau \& Rees, 2001), or perhaps (3) the coalescence of stellar remnants inside dense clusters (e.g. Quinlan \& Shapiro, 1990; Lee, 1995; Ebisuzaki et al., 2001). The subsequent growth of SMBH seeds is indirectly observed in galaxies containing active nuclei (or AGNs), which are thought to be powered by accreting SMBHs (e.g Lynden-Bell, 1969). In the local universe, gas and stellar kinematics in the nuclei of quiescent (non-AGN) galaxies indicate a population of dormant SMBHs as well, suggesting that AGN are a transient phase in the lifetimes of most galaxies 
(e.g. Soltan, 1982; Yu \& Tremaine, 2002).

\subsection{Feedback from Active Galactic Nuclei}

Outflows from AGN potentially play a very significant role in the evolution of large-scale structure. Such outflows consist of hot, tenuous out-flowing gas detected in absorption, and the powerful radio jets detected in some quasars. Blueshifted absorption lines (relative to systematic velocity of the AGN), such as Ly $\alpha$ and the $\mathrm{C}$ IV and $\mathrm{N}$ V doublets, indicate gas moving away from the central source at high velocities (Ulrich, 1988; Crenshaw et al., 1999). Broad absorption lines (BAL) indicating even higher velocities, are detected in luminous AGN (Hewett \& Foltz, 2003, e.g). Radio-loud quasars (RLQ) also carry substantial amounts of energy into the intergalactic medium (IGM) via collimated jets of relativistic plasma (e.g. Begelman et al., 1984). AGN outflows may play a role in distributing magnetic fields into the IGM (e.g. Furlanetto \& Loeb, 2001, hereafter FL01), and they can impact the evolution of their host galaxies by, for example, regulating the growth of supermassive black holes (Wyithe \& Loeb, 2003). BAL outflows and RLQ are also possible mechanisms for heating the intra-cluster medium (e.g. Valageas \& Silk, 1999; Nath \& Roychowdhury, 2002). The magnitude of the influence of outflows on the IGM depends on a few key properties of AGN, such as the relationship between kinetic and bolometric luminosity, which still need to be constrained by observation.

In RLQ, the kinetic luminosity of the jet is thought to be correlated with the bolometric luminosity (Willott et al., 1999). Direct estimates of the kinetic luminosity in BAL outflows can be made if such quantities as the covering fraction of the outflows, the outflow velocity, the radius of the outflow, and the column density are known. The velocities are accurately obtained from the absorption lines, and the covering fractions can be estimated with statistical arguments (Weymann, 
1997). The radius of the outflow can be obtained through observations of the photoionizing flux of the central source (Krolik, 1999). Estimates of the column densities can be made by studying the UV and X-ray absorption lines (Gallagher et al., 1999, 2001). Uncertainties in the above quantities translate to uncertainties in the kinetic luminosity, and therefore into uncertainties in the energy of AGN outflows. More energetic outflows can potentially fill a larger volume, having a greater impact on large-scale structure.

The colossal energy input from AGNs may also affect the clustering of matter. AGN outflows are known to influence their environments out to megaparsec scales, creating bubbles of hot, tenuous gas around their host galaxies. Evidence suggests that feedback processes, likely triggered by galaxy mergers, might play important roles in regulating star formation and quasar activity in their host galaxies as well as in neighboring galaxies, ultimately affecting the distribution of quasars (Di Matteo et al., 2005; Mahmood et al., 2005; Springel et al., 2005; Vittorini et al., 2005; Hopkins et al., 2006; Marulli et al., 2006). Additionally, outflows from AGN and starbursts, contribute significantly to the chemical enrichment and heating of the intergalactic medium (IGM) (e.g. Valageas \& Silk, 1999; Madau et al., 2001; Mori et al., 2002; Bromm et al., 2003; Wada \& Venkatesan, 2003; Fujita et al., 2004; Scannapieco \& Oh, 2004). With kinetic energies corresponding to as little as a percent of their bolometric luminosities, AGNs could produce outflows energetic enough to fill large fractions of the IGM with very low density bubbles (see, e.g., Chap. 2). Such bubbles would effectively push gas aside, influencing the distribution of baryons out to large scales and simultaneously impacting the subsequent development and evolution of neighboring structures (Scannapieco et al., 2002, 2005). If AGN outflows are energetic enough to influence the large scale distribution of baryons, it is possible that descriptions of the matter distribution on these scales will need to include them. 
Cosmic shear from weak lensing depends on the matter distribution on all scales, whether the matter is composed of baryons or dark matter. Because it depends on the mass distribution alone, it is also independent of the dynamics of the matter. Weak lensing can offer constraints on the equation of state of dark energy, as it is influenced by the expansion history of the universe. In order to use weak lensing to distinguish between different dark energy models, constraints on cosmological parameters determined from high precision measurements of the matter power spectrum require accurate modeling of the physics on the relevant scales (e.g. Annis et al., 2005). Hagan et al. (2005) have shown the importance of resolving dark matter substructure for simulating the nonlinear power spectrum. Baryons are assumed to closely follow the underlying dark matter distribution on large scales, however, on smaller scales baryons are taking part in more complicated physical processes which can result in a signal comparable to, if not larger than the statistical errors on the matter power spectrum (White, 2004; Zhan \& Knox, 2004; Jing et al., 2006; Rudd et al., 2008). Resulting discrepancies between weak lensing and other methods of determining cosmological parameters may simply be due to statistical fluctuations, but might also reflect the complicated role the baryons can play in shaping the matter power spectrum at the $10 \%$ level.

There is potentially enough energy available in the form of AGN feedback to influence structure out to cosmological scales, but whether feedback is likely to have such an impact depends first on how AGN interact with their own host galaxies. The energy in winds or jets can be dissipated within the host galaxy, and the host galaxy also dictates the fueling of AGNs. Since AGN are driven by accreting SMBHs, it is sensible to first model the fueling of SMBHs and the role of the small-scale physics within the host galaxy in sustaining that fueling. 


\subsection{Coevolution of SMBHs and Their Hosts}

It is increasingly evident that the growth histories of different components of galaxies-stars, gas, and supermassive black holes (SMBHs)-are intricately connected. Observations indicate a relationship between the masses of SMBHs and various properties of their host galaxies, such as the spheroid mass (Magorrian et al., 1998) and the velocity dispersion of stars in the bulge (Ferrarese \& Merritt, 2000; Gebhardt et al., 2000; Tremaine et al., 2002). Semi-analytic models and recent simulations have shown that feedback from accreting SMBHs can regulate the growth of the black holes, as well as the evolution of their host galaxies, making feedback a potentially important piece of the theory of SMBH-host galaxy co-evolution (e.g. Silk \& Rees, 1998; Kauffmann \& Haehnelt, 2000; Wyithe \& Loeb, 2003; Di Matteo et al., 2005; Springel et al., 2005; Di Matteo et al., 2007; Hopkins et al., 2007; Sijacki et al., 2007).

Building up the mass of a galaxy and driving AGN feedback requires a continuous replenishment of fuel in the center of the galaxy. A comprehensive understanding of such fueling is not possible without detailed knowledge of matter transport from large scales to the vicinity of the black hole. That transport helps determine, in particular, both the amount of material available for accretion, feedback, and star formation and the strength and duration of the fueling. Large-scale gravitational tidal fields created during major mergers of galaxies are thought to be effective at funneling matter toward the centers of galaxies (e.g. Hernquist, 1989; Barnes \& Hernquist, 1992). Indeed, simulations and semi-analytic modeling of hierarchical growth scenarios have shown that a combination of accretion and black hole mergers can effectively build up the local black hole population (Kauffmann \& Haehnelt, 2000; Yoo \& Miralda-Escudé, 2004; Volonteri \& Rees, 2005; Malbon et al., 2006; Volonteri \& Rees, 2006; Li et al., 2007b), as well as 
assemble the $\sim 10^{9} M_{\odot}$ black holes already observed to be present in quasars at $z \approx 6$ (Fan et al., 2003; Li et al., 2007b).

Secular evolution of galaxies, particularly in the absence of major merger events, can play an important role as disk instabilities lead to the formation of bars and spiral waves which can transport material. Global bar instabilities are thought to be efficient at transporting angular momentum, allowing material in the disk to move toward the center of the galaxy (Roberts et al., 1979; Simkin et al., 1980; Noguchi, 1988; Shlosman et al., 1989; Kormendy \& Kennicutt, 2004; Regan \& Teuben, 2004). In the "bars within bars" scenario of Shlosman et al. (1989), a large-scale galactic bar drives gas inward, where it forms a self-gravitating disk. As this disk becomes unstable, a smaller secondary bar forms, driving material down to scales of order $10 \mathrm{pc}$, at which point other physics can take over and transport the material the rest of the way toward the black hole (e.g. Shlosman et al., 1990).

There exist several observations of inflowing gas in the circumnuclear regions of low-luminosity AGN, apparently driven by global instabilities arising from secular evolution (e.g., see recent work by García-Burillo et al., 2005; Boone et al., 2007; Storchi-Bergmann et al., 2007; Lindt-Krieg et al., 2008; Riffel et al., 2008). Mergers are thought to be more important for building SMBHs in more massive early type galaxies (see e.g. Hopkins et al., 2008b,a, and references therein), ultimately building the SMBH-bulge relationships, whereas secular evolution may play a larger role in the growth of late type galaxies, particularly at lower redshift (see e.g. Kormendy \& Kennicutt, 2004, and references therein).

Whichever is the dominant mechanism for fueling SMBHs, the specific details of how fueling operates in the circumnuclear regions of galaxies are still not understood. Fueling may occur continuously, over the course of some large-scale dynamical instability in the galaxy, or it may be an intermittent process, depen- 
dent entirely on the dynamics on small scales. Cold gas, stochastically accreted from the circumnuclear regions of galaxies, may be able to sustain the fueling of low-luminosity AGN (Hopkins \& Hernquist, 2006). Other authors have suggested that intermittent accretion episodes, consisting of infalling clouds of gas with randomly oriented angular momentum vectors, may also contribute to the spin-down of SMBHs, thus lowering their accretion efficiency ${ }^{1} \quad$ (see King \& Pringle, 2006, 2007; King et al., 2008). Therefore, understanding the behavior of angular momentum in the circumnuclear region is an important part of characterizing the accretion rate.

\section{$1.4 \quad$ Numerical Progress}

Recently, cosmological smoothed particle hydrodynamics (SPH) simulations have been combined with smaller scale simulations of a SMBH host galaxy environment to study the effects of merger-driven fueling and AGN feedback on SMBH growth and demography (Di Matteo et al., 2007; Sijacki et al., 2007; Colberg \& Di Matteo, 2008). Reaching a large dynamic range in $N$-body $+\mathrm{SPH}$ simulations, Mayer et al. (2007) have studied galaxy dynamics during mergers resulting in a SMBH binary. Simulations which follow the growth of SMBHs over cosmological times, or over the duration of a galaxy merger, often cannot follow the circumnuclear regions of galaxies with high enough resolution to describe the accretion flow in detail. Such simulations must make approximations for the accretion rates using the properties of the galaxies which are resolved. A common technique is to assume Bondi accretion (Hoyle \& Lyttleton, 1939; Bondi \& Hoyle, 1944; Bondi, 1952), as in for example Di Matteo et al. (2005, 2007) who calculate the accretion rate on a scale of $100 \mathrm{pc}$, and $\mathrm{Li}$ et al. (2007a) who calculate the accretion rate

\footnotetext{
1 Conventionally, "accretion efficiency" describes the efficiency with which the rest mass energy of the accreted material is converted into radiation. Therefore, a lower accretion efficiency means that more mass is accreted.
} 
at 50pc. The assumption appears to be reasonable for following evolution over cosmological times, however for detailed studies of individual SMBHs or AGN, a more accurate estimate of the accretion rate is desirable.

Small-scale simulations have addressed gas dynamics in subgalactic-scale disks with high resolution (Fukuda et al., 2000; Wada, 2001; Wada \& Norman, 2001; Escala, 2007; Wada \& Norman, 2007), finding the development of a turbulent, multi-phase interstellar medium (ISM). The approximation of Bondi accretion in such an environment is not necessarily invalid. Krumholz et al. (2006) have shown that a modified form of the Bondi prescription can describe accretion in turbulent environments, however a simulation must be equipped to model the properties of the turbulence. Simulating both the complex physics in the ISM and the cosmological evolution of a galaxy, simultaneously, requires the use of adaptive techniques.

We have begun to address the issue of SMBH fueling using state-of-the-art cosmological simulations which are ideally suited to meet the numerical challenges presented above. The simulations use the adaptive mesh refinement (AMR) technique to self-consistently model the gas dynamics in a single galaxy at high resolution (subparsec resolution in the center of the galaxy). A large dynamic range (> 10 million), achievable with the AMR technique, allows us to bridge cosmological scales to scales relevant for molecular cloud formation (the birthplace of stars) and AGN fueling. It is a complex task to implement mergers, feedback, and secular evolution in large, cosmological simulations all at once. Our approach is to split the problem into pieces to be addressed one at a time, ultimately building a more realistic simulation. After studying the physics in this basic model galaxy, we can begin to include physical processes that are directly relevant to the problem of SMBH growth in the context of galaxy evolution, such as AGN feedback. 
The main goals of the work presented in this thesis are to address the following,

- What are the impacts of AGN outflows on cosmological scales?

- Do AGN outflows affect the interpretation of cosmological parameters?

- What drives mass transport within the circumnuclear regions of galaxies?

- How is the accretion rate in this region characterized?

- What is the angular momentum of gas on small scales?

The subsequent chapters can be divided into two categories: (1) A semianalytic assessment of the impact of AGN outflows on cosmological scales, and (2) A detailed study of the transport of gas and angular momentum in the circumnuclear regions of galaxies, modeled in a cosmological context. Chapters 2 and 3 address the former, and Chapters 4-6 address the latter. The results presented in Chapters 2, 3, and 5 have been published previously in the Astrophysical Journal (Levine \& Gnedin, 2005, 2006; Levine et al., 2007). 


\section{Chapter 2}

\section{AGN OUTFLOWS IN A COSMOLOGICAL CONTEXT}

In this chapter,we model the growth of AGN outflows using energy conservation arguments and simple approximations about their geometry. Through numerical simulations we model the distribution of these outflows and hence we can estimate the degree to which they affect the universe on global scales, or the fractional volume occupied by AGN outflows. In $\S 2.1$, we describe some of the details of the cosmological simulation and the luminosity function that we have

combined to obtain an AGN distribution. In $\S 2.2$ we explain the assumptions surrounding our physical model of the growth of heated bubbles around AGN as well as our selection of kinetic luminosity. In $\S 2.3$, we show the advantage of using a simulated density distribution over using simple statistical methods for the distribution of AGN. In $\S 2.4$ we examine the effects of simulation size and resolution, AGN lifetime, and AGN bias on the cumulative effect of outflows, and we conclude in $\S 2.5$. The rest of chapter adopts $\Omega_{m}=0.27, \Omega_{\Lambda}=0.73$, and $\Omega_{b}=0.04$ with $\Omega_{b} h^{2}=0.02$, consistent with the WMAP data.

\subsection{Simulating the AGN Environment and Distribution}

In order to understand the influence of AGN outflows on a global scale, it it useful to model the outflows in the context of large-scale structures. We assume here that AGN trace high density regions, and so use a gas density distribution 
to bias AGN in our study. Other studies have, for example, approximated the distribution of AGN with statistical formulae. Tegmark et al. (1993) and FL01 both use Poisson distributions to model the spatial distribution of galaxies for simplicity, in order to obtain filling fractions of supernova-driven winds and of AGN outflows respectively. We combine a z-dependent luminosity function with a simulated gas density distribution, allowing us to consider the AGN in their appropriate environments rather than homogeneously distributing them throughout the universe. In $\S 2.3$, we compare a Poisson distribution of AGN outflows with our model.

\subsubsection{Cosmological Density Distribution}

In order to model the evolution of the gas density distribution in the universe, we use a standard Particle-Mesh code to simulate the distribution of the dark matter, and we assume that on the scales we are considering, the gas distribution follows that of the dark matter, which is supported by cosmological gas dynamics simulations (Gnedin, 2000; Chiu \& Ostriker, 2000; Miller \& Ostriker, 2001; Somerville, 2002; Tassis et al., 2003; Benson \& Madau, 2003; Susa \& Umemura, 2004; Shapiro et al., 2004; Mo \& Mao, 2004). We also assume that the temperature of the cosmic gas is constant at 15,000 K. This assumption is, clearly, an oversimplification, since the temperature of the cosmic gas is known to evolve with time (Ricotti et al., 2000; Schaye et al., 2000; McDonald et al., 2001; Kim et al., 2002; Theuns et al., 2002; Hui \& Haiman, 2003) and vary in space. However, since the typical sizes of AGN-driven bubbles are significantly larger than the scales over which the temperature of cosmic gas changes, this approximation is sufficient for our purposes. 


\subsubsection{Luminosity Function}

The above simulation produces a gas density distribution at each time step, from $z \sim 19$ to $z=0$. In each step, we use a luminosity function to populate the simulation with AGN over a range of luminosities. In order to place AGN into our simulation, we must implement an AGN luminosity function that applies to high redshifts. The scarcity of high-z galaxy detections in surveys makes luminosity function predictions difficult, although the ability to make detections is improving. We use a model by Schirber \& Bullock (2003) for the QSO luminosity function. The model satisfies all existing constraints from optical surveys, such as the Sloan Digital Sky Survey (SDSS) data and the Great Observatories Origins Deep Survey (GOODS) data for $z>3$ (Fan et al., 2001a,b; Cristiani et al., 2005) and Two Degree Field (2dF) data for $z<2.3$ (Boyle et al., 2000). It should be noted that optical surveys perhaps underestimate the faint-end, low-z luminosity function. Hard X-ray detections of AGN yield a higher number of faint AGN at low-redshift than in previous optical surveys (Barger et al., 2005). The model that we use for now parameterizes the following luminosity function:

$$
\phi\left(L_{B}, z\right)=\frac{\phi_{*} / L_{*}}{\left(L_{B} / L_{*}\right)^{\gamma_{f}}+\left(L_{B} / L_{*}\right)^{\gamma_{b}}} .
$$

In the above equation, $\phi_{*}$ is the average comoving number density of AGN, $L_{*}$ and $L_{B}$ are the break luminosity and AGN B-band luminosities respectively (given in units of $L_{\odot, B}$ where we have followed SB03 in using $2.11 \times 10^{33} \mathrm{ergs} \mathrm{s}^{-1}$ for the B-band luminosity of the sun), and $\gamma_{f}$ and $\gamma_{b}$ are the faint and bright-end slopes respectively. As in SB03, we interpolate the SDSS and 2dF fits for $2.3<z<$ 3. The parameterization is given in Table 2.1, and the luminosity function for several different redshifts is plotted in Figure 2.1. Note that for $z>3, L_{*}$ and $\phi_{*}$ depend on the weighted emissivity of AGN (accounting for contributions to 
Table 2.1. Parameterization of the luminosity function

\begin{tabular}{cccc}
\hline \hline Parameter & $z<2.3$ & $2.3<z<3$ & $z>3$ \\
\hline $\log \left(\phi_{\star} \mathrm{Gpc}^{3}\right)$ & 3.029 & $-0.87 z+5.02$ & $2.80+2.72 \log \hat{\varepsilon}^{Q}+0.81(z-3)$ \\
$\log \left(L_{\star} L_{\odot, B}^{-1}\right)$ & $11.24+1.36 z-0.27 z^{2}$ & $-0.72 z+14.6$ & $12.2-1.72$ \\
$\gamma_{b}$ & 3.41 & $-1.19 z+6.14$ & 2.58 \\
$\gamma_{f}$ & 1.58 & 1.58 & 1.58 \\
\hline
\end{tabular}

the ionizing rate from other sources, such as stars). The weighted emissivity is given by $\log \hat{\varepsilon}^{Q}=-0.245 z+0.596$, a simple power law fit to the values given in SB03 Table 1. We have chosen a minimum AGN luminosity of $10^{8} L_{\odot, B}$ as in SB03, who argue that this represents the faintest Seyfert galaxies found. We extrapolate the high-z parameterization of Table 2.1 to $z \sim 19$, which introduces a significant uncertainty in the abundance of AGN at high redshift. However, as we show below, AGN at $z<3$ dominate, so this uncertainty affects our results insignificantly. The exact redshift range for each run depends on the size of the simulation box since larger boxes can sample an earlier, rarer population of AGN.

It should be noted that we used SB03's "Model A" to determine this particular luminosity function for $z \geq 3$. The model uses the emissivity of the ionizing background, assuming a stellar as well as an AGN contribution, to constrain the AGN luminosity function in combination with the SDSS data. The model implements the ionizing rates of McDonald \& Miralda-Escudé (2001) and assumes an escape fraction of 0.16 (including the stellar contribution to the ionizing background). The model neglects optically obscured AGN on the basis that they do not make a significant contribution to the ionizing background. This means that the luminosity function we use could be excluding some AGN that host outflows. Of the models described by SB03, we have chosen "Model A" because it is most consistent (of the models studied by SB03) with the faint end luminosity function 
(for $z>4$ ), from GOODS (Cristiani et al., 2005).

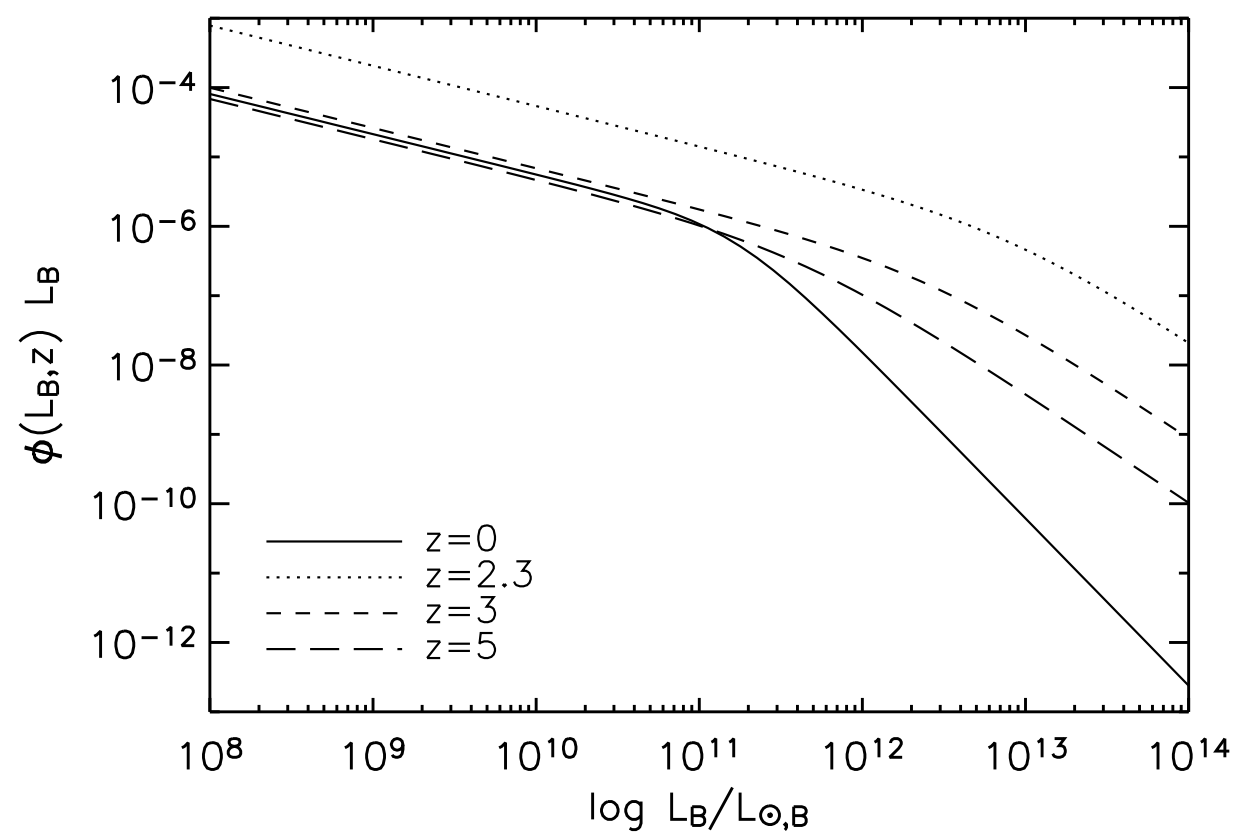

Figure 2.1 Calculated comoving number density of AGN at a given redshift, using a luminosity function consistent with $2 \mathrm{dF}$, SDSS and GOODS data.

We use the luminosity function to calculate the number of AGN at a given redshift and luminosity in each simulation box, and we then place each one into a random location, but with a bias toward high density regions. In order to distribute AGN so that they correspond to regions of high density, we calculate, for each cell, the probability of hosting an AGN given by

$$
P(i, j, k)=\frac{\rho_{m}^{\alpha}(i, j, k) \Delta V}{\sum_{i, j, k=0}^{N-1}\left(\rho_{m}^{\alpha} \Delta V\right)} .
$$

Above, $\rho_{m}$ is the matter density (in units of average baryon density) in each cell specified by coordinates $i, j, k ; N$ is the size of simulation box; $\Delta V$ is the comoving volume of each cell; and $\alpha$ is a bias parameter ensuring that regions of higher density are more likely to host AGN. The above probability function is independent 
of the characteristics of individual AGN (such as luminosity). Analysis of existing observations suggests that AGN are more biased toward high density regions at increasing redshifts (e.g. Porciani et al., 2004; Croom et al., 2005). In §2.4.3, we will examine the effects of different bias parameters on our results. In order to place the AGN in specific cells, we randomly choose a cell and compare the value of $P(i, j, k)$ in that cell to a randomly generated number between zero and one. If $P(i, j, k)$ is greater than the random number, an AGN goes into the cell. Otherwise, the process repeats until the AGN has been placed in the simulation. This method has the effect of biasing AGN toward regions of higher density, while fully allowing for Poisson noise in their distribution.

\section{$2.2 \quad$ AGN Outflows}

Sections 2.2.1, 2.2.2, and 2.2.3 detail the distribution and kinematics of AGN outflows in our model. Outflows are not observed in all AGN, and so §2.2.1 deals with our method of selecting AGN to host outflows. In $\S 2.2 .2$, we describe our assumptions about the expansion and evolution of individual outflows into the IGM, and in $\S 2.2 .3$, we discuss the effects of kinetic luminosity on the outflows.

\subsubsection{Distribution of Outflows}

In our model we assume that the AGN produce outflows that are responsible for distributing tenuous, hot gas into the IGM. The outflows may also be a mechanism for depositing metals and magnetic fields into the IGM. However, outflows are only associated with a fraction of AGN, which we must reflect in our model. Detection of blue-shifted broad absorption lines in an AGN's spectrum indicates out-flowing gas from the nucleus. Until fairly recently, observations indicated that these broad absorption lines were limited to radio-quiet quasars (Stocke et al., 1992), and only seen in $\sim 10 \%$ of them. However, recent detections 
of BAL outflows in RLQ (e.g. Brotherton et al., 1998; Menou et al., 2001) suggest that BAL outflows do not necessarily follow this radio dichotomy. There is, however, evidence for some luminosity dependence in the occurrence of BAL outflows. Crenshaw et al. (1999) examined the UV spectra of several Type I Seyfert galaxies obtained with the Hubble Space Telescope, and found intrinsic, narrow absorption lines in more than half of their sample. The absorption spectra of these low-luminosity objects show similarities to the BAL features in high-luminosity objects, suggesting a possible relationship between the two. On the higher luminosity end, Hewett \& Foltz (2003) apply a magnitude correction to the Large Bright Quasar Survey (LBQS Hewett et al., 1995, 2001) and find a higher percentage of BAL quasars than previously determined. Magnitude and flux-limited samples can exclude BAL quasars in which the absorbing gas reduces the spectral energy distribution of the quasars in the wavelength range of selection. After applying a correction for this effect, Hewett \& Foltz find that $\sim 20 \%$ of the AGN in the sample host outflows.

In our model, we interpolate the above low and high-luminosity limits for the fraction of AGN hosting outflows, $f_{\text {out }}$, and combine this fraction with the luminosity function of $\S 2.1 .2$. Our formulation is as follows:

$$
f_{\text {out }}= \begin{cases}.5 & \text { for } \log L_{B}<10 \\ 1.5-0.1 \log L_{B} & \text { for } 10 \leq \log L_{B} \leq 13 \\ .2 & \text { for } \log L_{B}>13\end{cases}
$$

where the lower and higher-end luminosities are consistent with the fractions determined above. It is not well known whether the above statistics also apply to the population of X-ray detected AGN without optical counterparts. However, because X-ray surveys detect more low-luminosity AGN than optical surveys, it 
is possible that there are more AGN containing outflows than we here assume. Additionally, it should be noted that because of the range of covering fractions of AGN outflows, the fraction of AGN hosting outflows could be larger than what is observed, even in optical surveys, due to orientation effects (Morris, 1988; Weymann et al., 1991; Hamann et al., 1993). Therefore, our assumptions should provide a conservative lower limit to the number of AGN hosting outflows.

\subsubsection{Evolution of Outflows}

In order to understand the degree to which active galaxies affect the IGM, it is important to have an accurate model of the expansion of hot, ionized gas into the IGM. The environment of the outflow must be considered in order to model the expansion. Using the density profile described in $\S 2.1$, we can, to a degree, reproduce the environment surrounding each of the individual active galaxies that we placed in our simulation in accordance with the luminosity function (eq. 2.1) and $f_{\text {out }}$ (eq. 2.3).

The lifetime of the active galactic nucleus is short compared to the expansion time of the bubbles, which lasts over the duration of our simulation. Therefore, we consider the active phase as a brief energy injection, followed by an adiabatic blast wave gathering up material in the IGM, analogous to the adiabatic phase of a supernova remnant. As in Scannapieco \& Oh (2004), we have used the familiar Sedov-Taylor blast wave model to determine the size of the bubbles in our analysis. Their Equation 10, which we adopt for convenience of units, follows:

$$
R_{s}=(1.7 \mathrm{Mpc})\left(\frac{E_{k}}{10^{60} \mathrm{ergs}}\right)^{1 / 5}\left(1+\delta_{m}\right)^{-1 / 5}(1+z)^{-3 / 5}\left(\frac{t_{\text {age }}}{10^{9} \mathrm{yr}}\right)^{2 / 5}
$$

In the above equation, $E_{k}$ is the kinetic energy injected by the AGN, $\delta_{m}$ is the overdensity, and $t_{\text {age }}$ is the time since the active phase began. The overdensity 
is an average over all cells within the radius of the bubble, obtained using an iterative technique. The above Sedov-Taylor solution, with a constant density, is clearly not valid as the outflow escapes its host galaxy and travels first through a region with a steeply-falling off density profile, e.g. the NFW profile (Navarro et al., 1997). However, our simulation does not resolve this stage of the expansion. The virial radius of a typical $L_{\star}$ galaxy is well below the resolution of our simulation. Without modeling the expansion of the outflows inside this region, there is uncertainty in the speed with which the outflows escape their host galaxies. However, this uncertainty is hidden by the parameter $E_{k}$, which describes the actual energy input from the AGN.

We assume that the bubbles expand according to the above equation until they reach a pressure equilibrium with their environment. If pressure equilibrium is reached before the energy injection has stopped, the growth of the bubbles is determined by the surrounding pressure and the injected energy, and the size is given by

$$
R_{P}=\left(3.24 \times 10^{-25} \mathrm{Mpc}\right)\left(\frac{3 E_{k}}{4 \pi P}\right)^{1 / 3} .
$$

The pressure of the surrounding medium is given by $P=\left(1+\delta_{m}\right) \bar{n}_{b} k_{b} T$, where the quantity $\left(1+\delta_{m}\right) \bar{n}_{b}$ is the average gas density inside the bubble as determined above, and $T=1.5 \times 10^{4} \mathrm{~K}$ (the average temperature of the IGM). The kinetic energy in Equations 2.4 and 2.5 is given by $E_{k}=\varepsilon_{k B} L_{B} t_{\text {age }}$ where $t_{\text {age }}$ is the age of the AGN during the active phase, or the lifetime of the AGN once the active phase has ended. We assume a constant lifetime of $10^{8} \mathrm{yrs}$ for all AGN until $\S 2.4 .2$ in which we will examine other lifetimes. The parameter $\varepsilon_{k B}$ is given by the ratio of the kinetic luminosity of the outflow to the AGN B-band luminosity $\left(L_{k} / L_{B}\right)$. Each AGN injects kinetic energy into the IGM at a rate $\left(L_{k}\right)$ correlated 
with the AGN's luminosity. We assume that the AGN are accreting at roughly their Eddington rates, so that $L_{e d d} \sim L_{b o l}$. As in FL01, we adopt $L_{b o l} \sim 10 L_{B}$, consistent with Elvis et al. (1994). We describe our choice of the kinetic fraction, $L_{k} / L_{b o l}$ or $\varepsilon_{k}$, in the next $\S 2.2 .3$.

After the energy injection phase, bubbles in pressure equilibrium no longer expand as a result of the energy injection of the AGN. Any subsequent evolution of the bubbles in our simulation is determined by the Hubble expansion and the evolution of the density distribution in the surrounding environment. Overlap of the bubbles is not likely to affect the expansion significantly. When an expanding bubble in pressure equilibrium overlaps with the interior of another bubble, encountering densities much lower than those typical in the IGM, the expansion speed does not change, as there is no longer a pressure gradient.

Our model does not include radiative cooling, as the cooling times for these bubbles are typically much longer than the timescales we consider. However, for bubbles located in cluster environments (higher densities and temperatures, etc.), radiative cooling, as well as other physical processes, may become important. Accurately modeling outflow physics in these complex environments requires the use of hydrodynamical simulations. Separate work is currently underway to understand the impact of AGN outflows in these environments (e.g. Brüggen \& Kaiser, 2002; Ruszkowski et al., 2004).

Figure 2.2 shows the evolution of the bubble size for two typical AGN (each with luminosities of $\sim 10^{9} L_{\odot, B}$ ) residing in different environments within the simulation. The figure shows that the AGN in the lower density environment produces a larger bubble $\left(\sim 4.8 h^{-1} \mathrm{Mpc}\right)$ than the AGN in the higher density environment $\left(\sim 2.2 h^{-1} \mathrm{Mpc}\right)$.

In both FL01 and Nath \& Roychowdhury (2002), AGN outflows are treated as collimated jets that spread out into a cocoon after reaching pressure equilib- 


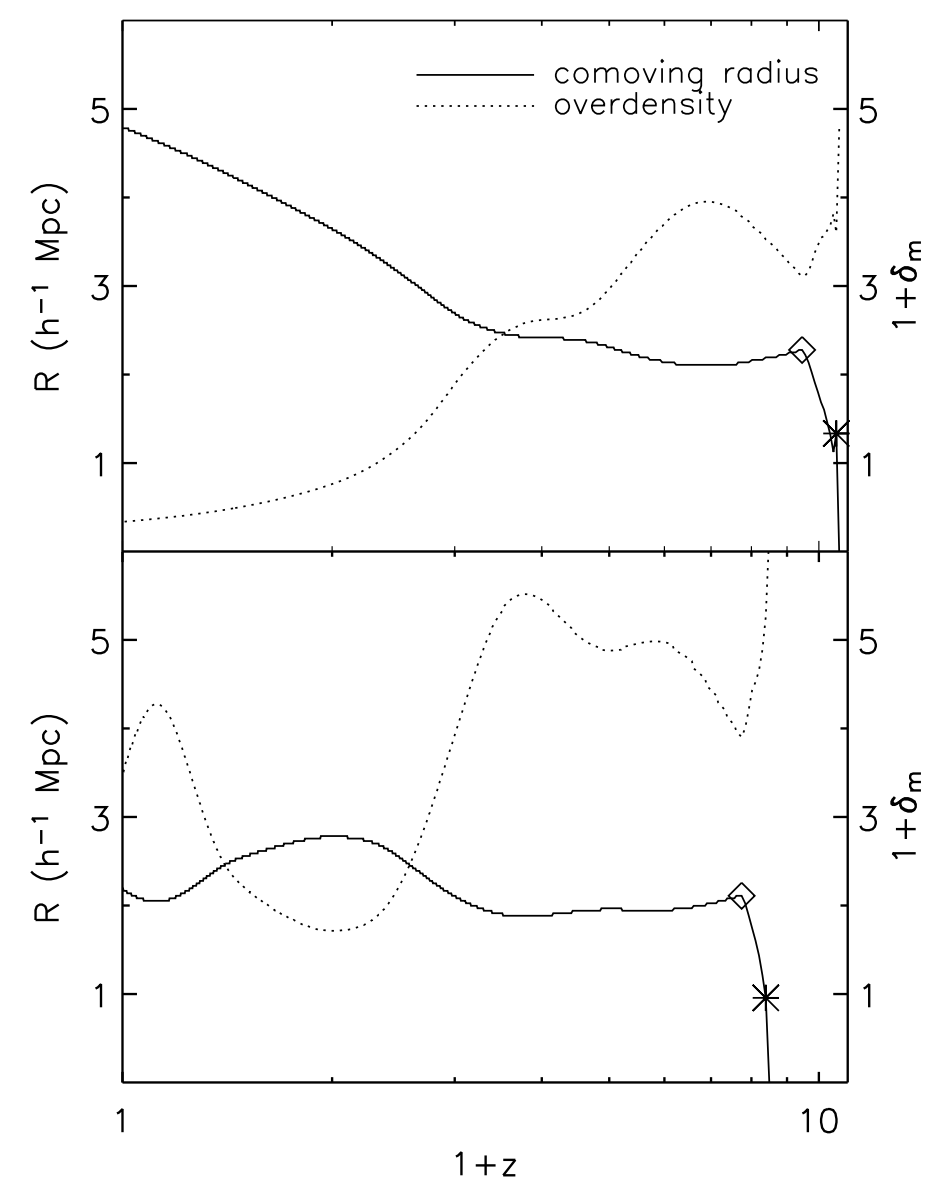

Figure 2.2 Overlay of the comoving radius of two AGN bubbles (solid) in different environments, and the average overdensity, $1+\delta_{m}$, inside each bubble (dotted). We have chosen AGN with luminosities of $\sim 10^{9} L_{\odot, B}$. We show the point at which the bubbles reach pressure equilibrium with their environments (star) and the end of the active phase after a lifetime of $10^{8} \mathrm{yrs}$ (diamond). The figures represent typical AGN, although the exact behavior is a function of luminosity and environment. Note that the continued growth of the bubble after reaching pressure equilibrium is a result of the continuing energy injection from the nucleus. Once the energy injection (active phase) ends, the bubble size only changes with the evolution of its environment and the Hubble flow. In the top figure, the bubble is expanding into a region of lower density, allowing the expansion to continue. In the bottom figure, the bubble expands into a denser region, eventually halting the expansion. 
rium at the end of the AGN's active phase. Furthermore, both BAL AGN and RLQ are treated similarly, justified by the small covering fraction of BAL outflows, averaging around 10\% (Weymann, 1997). We likewise adopt the practice of treating the two different objects similarly, noting that there is an additional justification for this in the likelihood of overlap between RLQ and BAL AGN. Furthermore, because the time scales we consider are significantly longer than the AGN injection phase, we do not model collimated jets, but rather approximate the outflows as bubbles expanding into the IGM with spherical symmetry. However, as demonstrated by Figure 2 of FL01, if the energy injection is modeled assuming spherical symmetry during the active phase, rather than with collimated jets, the result is only a slightly smaller final comoving bubble size. The entirely spherical case produces a smaller cocoon because the surface area of the bubble causes it to decelerate sooner, whereas a collimated jet makes its way through the IGM more easily.

\subsubsection{Kinetic Luminosity and Filling Fraction}

Estimates of the kinetic fraction, $\varepsilon_{k}$, are subject to a number of observational uncertainties surrounding BAL outflows. Observational constraints depend on quantities that are difficult to determine, such as the distance of outflows from the central source, and the covering fraction of the outflows (e.g. de Kool et al., 2001). We use our model of AGN outflows to calculate the filling fraction of outflows as a function of redshift, $F(z)$, for a box of length $128 h^{-1} \mathrm{Mpc}$ (with $0.5 \mathrm{~h}^{-1} \mathrm{Mpc}$ cells), assuming that all of the AGN are active for $10^{8} \mathrm{yrs}$, and that they follow a constant bias, $\alpha=2$. We then treat the kinetic fraction as a free parameter of our model. We start with $\varepsilon_{k B}=1$, or a kinetic fraction $\varepsilon_{k}=0.1$, as in FL01. NathRoy02 argue that $\varepsilon_{k}=0.1$ is probably an upper limit for BAL outflows, assuming that the covering fraction of BAL outflows is $\sim 10 \%$. Figure 


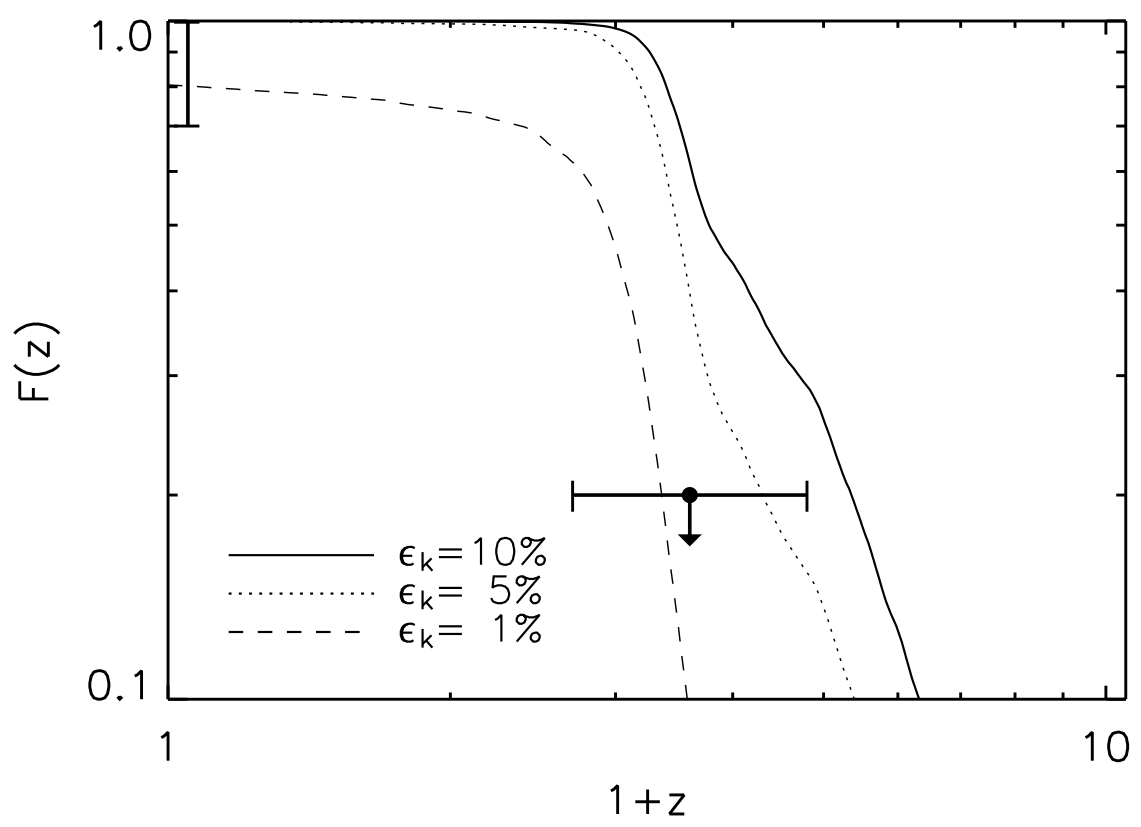

Figure 2.3 Fractional volume filled with AGN outflows as a function of redshift for different kinetic fractions. The bars represent the observational constraints on the filling fraction of AGN outflows from the Ly $\alpha$ forest, as discussed in the text.

2.3 shows the fractional volume filled with AGN outflows for decreasing kinetic fraction. We find that using a kinetic fraction of $10 \%$, the entire simulation box is filled with outflows by $z \sim 2$. Similarly, Scannapieco \& Oh (2004) find that a kinetic fraction of $10 \%$ overestimates feedback effects in their model of AGN outflows.

Knowledge of the filling fraction of the Ly $\alpha$ forest at various redshifts can provide further constraints, if we assume that the AGN outflows consist of hot, tenuous gas that cannot occupy the same volume of space as Ly $\alpha$ absorbing regions. At low-z, simple conclusions drawn from observations of the Ly $\alpha$ forest constrain the fractional volume of voids to between 70 and $99.6 \%$ of the total volume, providing an upper limit to the filling fraction of AGN outflows (e.g. Penton et al., 2004; Davé et al., 1999). The vertical bar near $z=0$ in Figure 2.3 shows the 
constrained filling fraction of voids. An $\varepsilon_{k}$ of less than $10 \%$ produces filling factors that are consistent with the above values, but imposing more precise constraints from Ly $\alpha$ forest observations is difficult because of the range of column densities under consideration. For somewhat higher redshifts $(1.7<z<3.8)$, Duncan et al. (1989) have studied voids in the $\operatorname{Ly} \alpha$ forest and determined that voids with sizes between 10 and $70 h^{-1} \mathrm{Mpc}$ occupy $<20 \%$ of the volume. The horizontal bar in Figure 2.3 shows this upper limit for the filling fraction of AGN outflows over the appropriate redshift range. We choose $\varepsilon_{k B}=.1\left(\varepsilon_{k}=1 \%\right)$ as our fiducial value, as it seems to match observational and theoretical constraints more closely than the higher values.

\subsection{Comparison with a Poisson Distribution}

As previously mentioned, it is possible to calculate the filling fraction of AGN outflows analytically by assuming that AGN are distributed according to Poisson statistics. In this section, we demonstrate the advantage of including a realistically evolving density distribution in our model.

We calculate the filling fraction of outflows using a Poisson distribution of sources and compare with our method of biasing AGN toward the high density regions within a cosmological simulation (see $\S 2.1$ ). We first calculate the porosity of AGN outflows at each redshift, given by:

$$
Q(z)=\frac{4 \pi}{3} \int_{z}^{\infty} \frac{d z^{\prime}}{\tau_{A G N}} \frac{d t^{\prime}}{d z^{\prime}} \int_{L_{\min }}^{L_{\max }} R^{3} \phi\left(L_{B}, z\right) f_{\text {out }} d L_{B}
$$

where the above quantities are calculated in physical units. We then calculate the filling fraction assuming a Poisson distribution:

$$
f(z)=1-e^{-Q(z)} .
$$


In the above calculations, we have determined the volume of each outflow under pressure equilibrium conditions, and under the assumption that the energy injection is instantaneous. The pressure is determined by the average particle density at each redshift and a constant temperature of $T=1.5 \times 10^{4} \mathrm{~K}$. We compare the above filling fraction with that produced by our model, as described in $\S 2.2 .3$, for a simulated volume of $128^{3} h^{-3} \mathrm{Mpc}^{3}$ (with $0.5 h^{-1} \mathrm{Mpc}$ cell resolution) in Figure 2.4. The Figure shows that the Poisson distribution of sources produces a higher filling fraction than our model. The simulation provides a realistic environment for each AGN. The outflows of AGN residing in regions of higher density do not fill as large a volume because the IGM exerts more pressure on the bubbles than in a uniform, average density distribution. Also, the AGN themselves are not distributed over as large a volume of space, as they tend to be located in higher density regions. Therefore, their outflows do not fill as large a fraction of the simulation as if they were uniformly distributed.

\section{$2.4 \quad$ Results}

Sections 3 and 4 show the general result of our model on the filling fraction of AGN outflows. In this section, we will examine the effects of varying simulation box size and resolution, AGN lifetime, and the distribution of AGN as determined by AGN bias. We will first conduct convergence studies, to determine the effects of box size and resolution on the rest of our analysis.

\subsubsection{Convergence Studies}

We ran our analysis on simulations of differing box sizes in order to choose the optimal box size for the rest of our studies. Larger boxes are more inclusive, but also much more computationally expensive, and so convergence is desirable. For each box size, we determine the fractional volume heated by AGN as a function 


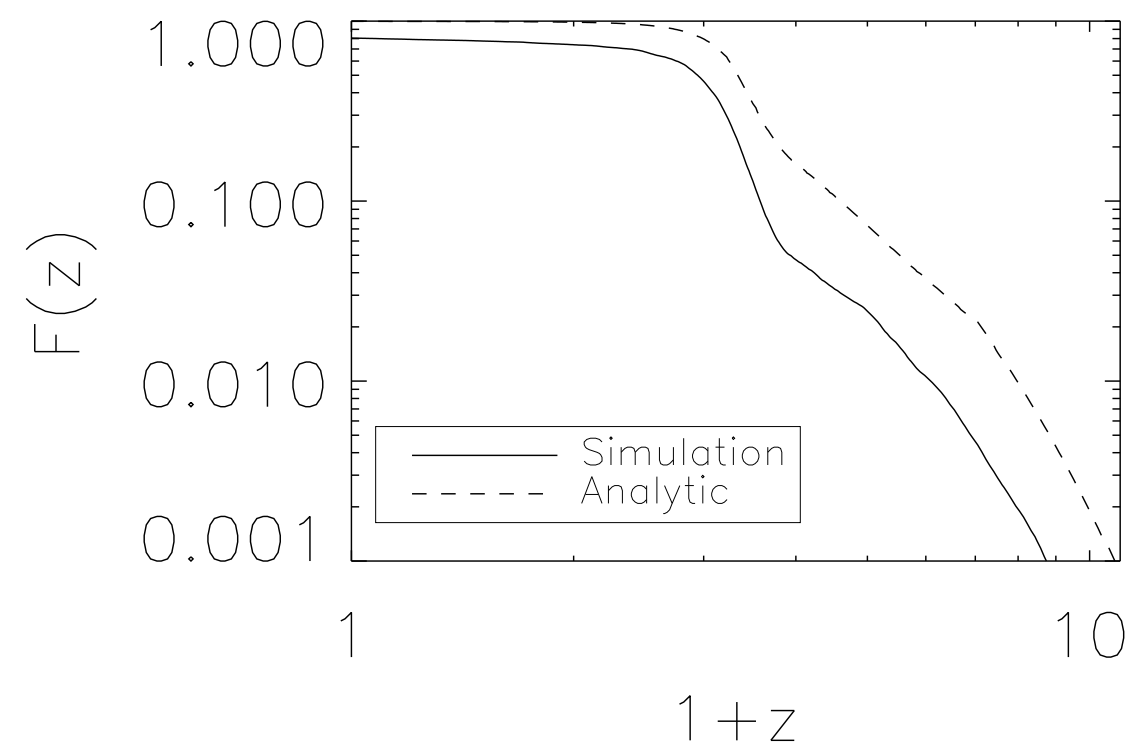

Figure 2.4 Filling fractions for a uniform density distribution (no density bias) and for an evolving density distribution (with density bias). The simple Poisson distribution results in a higher filling fraction than in the scenario accounting for an evolving density distribution.

of redshift using the model for bubble growth described in $\S 2.2$. We have evolved boxes of length $64 h^{-1} \mathrm{Mpc}, 128 h^{-1} \mathrm{Mpc}$, and $256 h^{-1} \mathrm{Mpc}$ (each with $1 h^{-1} \mathrm{Mpc}$ cells) on a side. Figure 2.5 shows the filling fraction for each box size. The filling fraction does not vary dramatically between the two larger box sizes (for this reason, we did not complete the run for the largest, most expensive box size). It appears that we have reached convergence for the $128 h^{-1} \mathrm{Mpc}$ box. We therefore choose the $128 h^{-1} \mathrm{Mpc}$ box size for all of our parameter studies.

In addition to box size studies, we also examined the effects of simulation resolution on the AGN heated fractional volume. In simulations with finer resolution, individual cells can reach significantly higher densities (see Fig. 2.6), which directly affects the sizes of the bubbles in our simulations. The result is a different fractional volume affected by AGN depending on resolution. High resolution gen- 


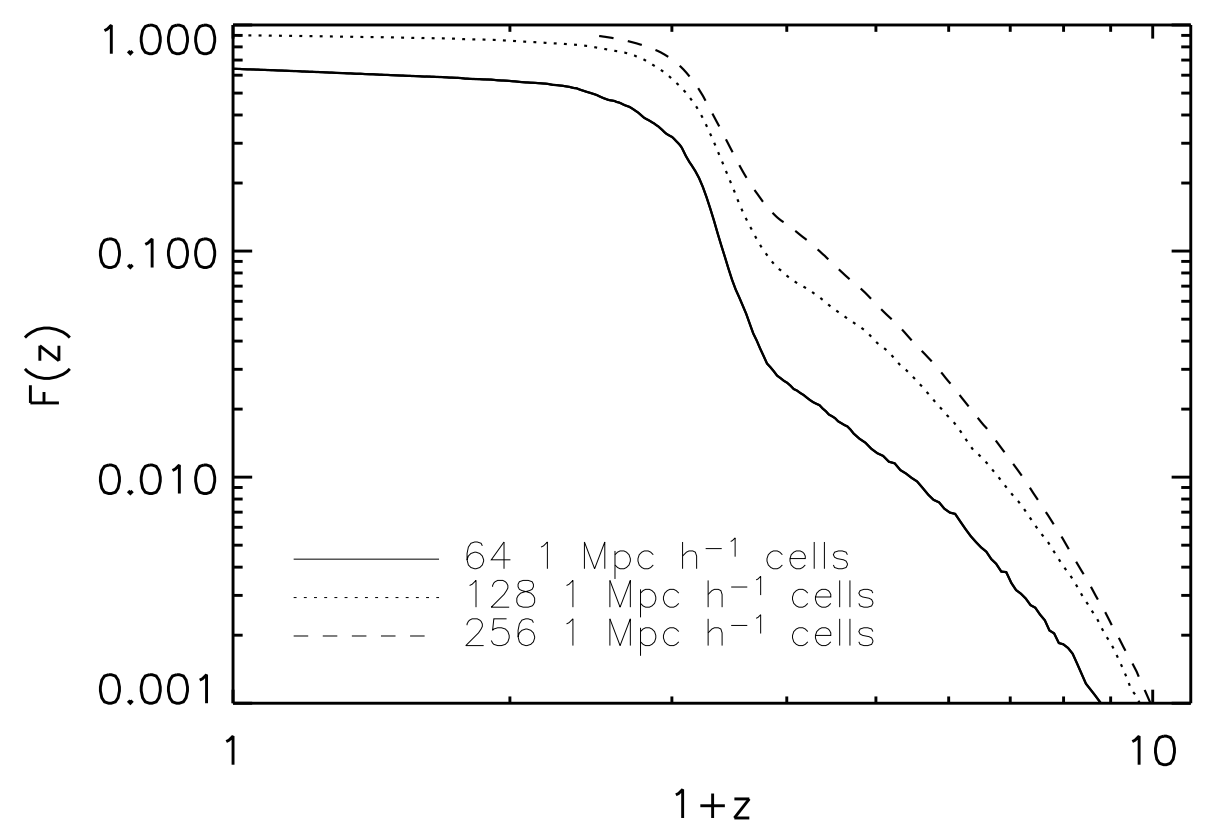

Figure 2.5 Filling fractions for different box volumes. Note that due to the computation time required for the $256 h^{-1} \mathrm{Mpc}$ length box, we did not calculate the filling fraction all the way to $z=0$.

erates a more accurate picture of the simulated volume, but like large box size, is more computationally expensive because of the larger number of cells. Therefore, we increase the resolution in our simulation box until the filling fraction no longer depends on resolution, after which, there is no need for finer resolution. We studied the $64 h^{-1} \mathrm{Mpc}$ length box with resolutions of $1 h^{-1} \mathrm{Mpc}$ cells, $0.5 h^{-1} \mathrm{Mpc}$ cells, and finally $0.25 \mathrm{~h}^{-1}$ Mpccells. We reach convergence in the filling fraction very quickly, as shown in Figure 2.7. The results for the $0.5 h^{-1} \mathrm{Mpc}$ cells and the $0.25 h^{-1} \mathrm{Mpc}$ cells are very similar, and so we choose the faster computation, $0.5 h^{-1} \mathrm{Mpc}$ cells, for our fiducial resolution. 

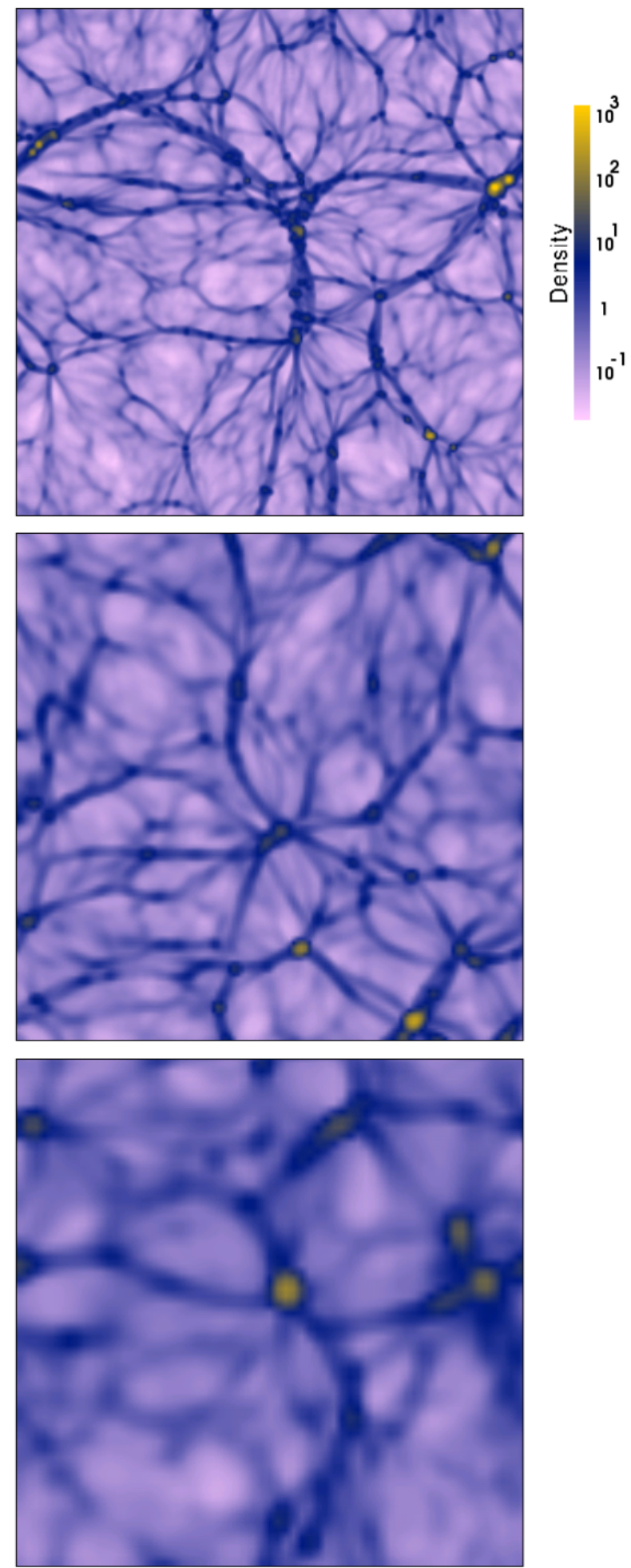

Figure 2.6 Examples of different resolutions. The above Figures show cross sections of the density distribution at $\mathrm{z}=0$ for runs with differing resolutions. The upper box has $0.25 h^{-1} \mathrm{Mpc}$ cells, the center box has $0.5 h^{-1} \mathrm{Mpc}$ cells, and the lower box has $1 h^{-1} \mathrm{Mpc}$ cells. All boxes are $64 h^{-1} \mathrm{Mpc}$ across. A wider range of densities is reached for finer resolved boxes. 




Figure 2.7 Filling fraction for different cell resolutions of a box of length $64 \mathrm{~h}^{-1} \mathrm{Mpc}$. The two most finely resolved runs produce very similar results by $z=2$, and so we choose $0.5 h^{-1} \mathrm{Mpc}$ cells as our fiducial resolution, as it is the more computationally inexpensive of the two.

\subsubsection{AGN Lifetime}

We have chosen for our fiducial model a constant lifetime for all AGN, $\tau_{A G N}=10^{8}$ yrs. Yu \& Tremaine (2002) examine the dependence of lifetime on black hole mass and their results show modest variation in lifetime $(\sim 30-$ $300 \mathrm{Myr}$ ) over the range of black hole masses of interest here. They determine AGN lifetime from a combination of the luminosity function and black hole number density, and their results are comparable to the associated Salpeter times, further evidence that black holes accrete most of their mass during their active phases. Our choice of $\tau_{A G N}=10^{8} \mathrm{yrs}$ is consistent with their results. We test the effect of using higher and lower lifetimes as well. We apply a constant lifetime of $10^{7} \mathrm{yrs}$ as our lower limit. According to SB03, $10^{7} \mathrm{yrs}$ is an approximate 
lower limit for AGN lifetime, obtained from arguments similar to those of $\mathrm{Yu} \&$ Tremaine (2002):

$$
f_{\text {on }}(z)=\frac{\tau_{A G N}}{t_{\text {Hubble }}} \geq \frac{\Phi\left(>L_{B}, z\right)}{n\left(>M_{B H}, z=0\right)} .
$$

In the above equation (eq. 22 of SB03), the AGN lifetime is determined from the fraction of galaxies having active nuclei at a given redshift $\left(f_{\text {on }}\right)$. This fraction can be determined by the ratio of the comoving number density of AGN (where $\Phi$ is the number density of AGN with luminosities greater than $L_{B}$ at redshift $z$ ) to the black hole number density $(n)$. SB03 assume that the black hole number density only increases with time, so that eq. 2.8 , with the number density evaluated at $z=0$, will give a minimum lifetime. For our upper limit, we use $\tau_{A G N}=10^{9} \mathrm{yrs}$, consistent with Croom et al. (2005) who determine an upper limit on AGN lifetime from arguments about the growth of dark matter halo mass. We do not examine redshift or mass dependence of $\tau_{A G N}$, because the variation is not significant over the range of lifetimes we consider.

We find that shorter lifetimes result in a lower filling fraction. In order to remain consistent with the luminosity function in the case of shorter lifetimes, many more AGN will become active at later redshifts than in the longer lifetime case. As AGN are born later in the simulation, they sit in regions of higher density than AGN born earlier, as the density distribution evolves with redshift. In this analysis, we have not included the possibility of recurrent activity associated with the same nucleus because the duty cycles of AGN are so low that it would introduce a small effect. Figure 2.8 shows $F(z)$ for lifetimes of $10^{7}, 10^{8}$, and $10^{9}$ yrs. In order to test that our interpretation of the effect of evolving density distribution is correct, we calculate $F(z)$ for the three values of AGN lifetime under the assumption that the density distribution is uniform throughout the universe. Figure 2.9 


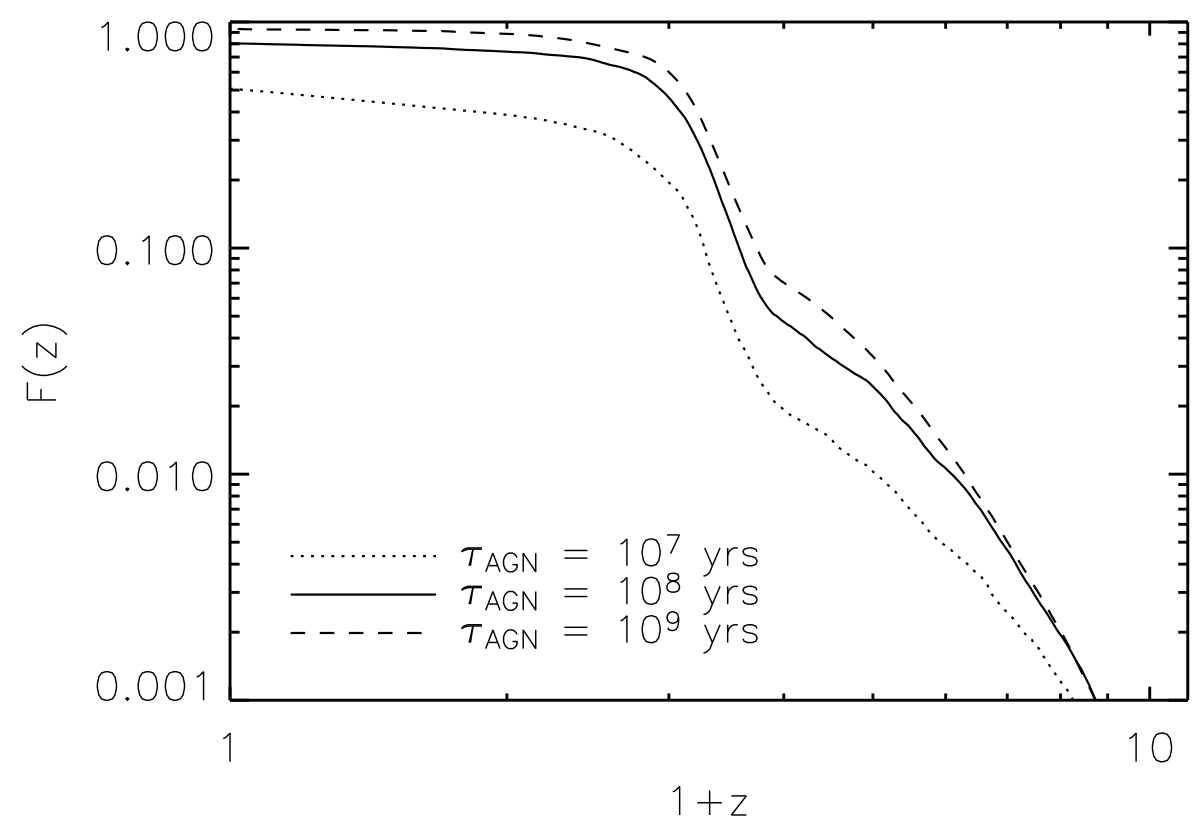

Figure 2.8 Filling fraction for different values of constant AGN lifetimes. Shorter AGN lifetimes imply younger AGN living in higher density environments.

shows the same range of lifetimes for the uniform density case. As expected, here we do not see the trend with lifetime because the density dependence has been removed. Therefore, because of the evolving density distribution, a shorter AGN lifetime will result in a lower filling fraction at $z=0$.

\subsubsection{AGN Bias}

Observations show that AGN are biased toward regions of high density, with this bias increasing toward higher redshifts. A simple, quantitative implementation of this observed bias is the relation $n_{A G N} \propto \rho_{m}{ }^{\alpha}$, where $n_{A G N}$ is the number density of AGN in units of average number density of AGN, and $\alpha$ is the linear bias parameter. Therefore, $\alpha$ is a measure of the correlation between matter density and AGN density. We examine simple constant bias models here as well as one redshift dependent model. We do not, however, examine the possibility of lumi- 


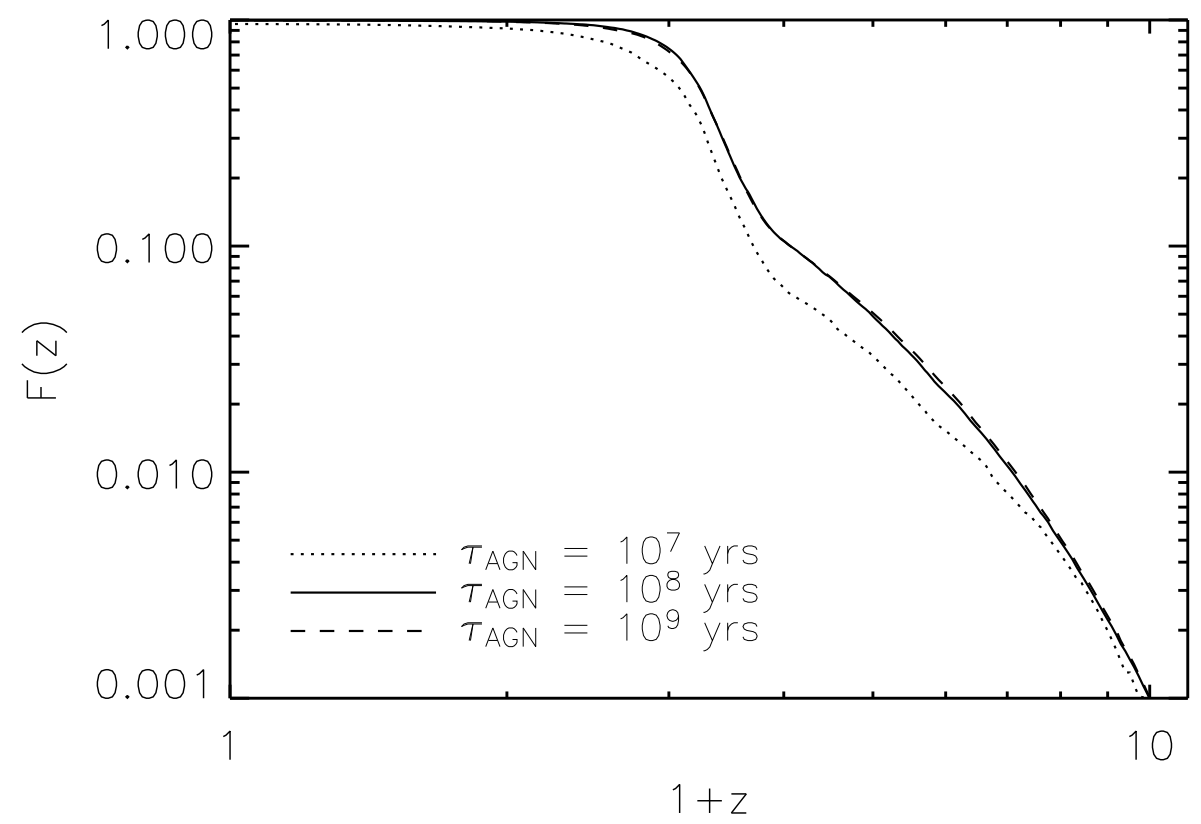

Figure 2.9 Filling fraction for different values of constant AGN lifetimes for a constant, isotropic density distribution. Without an evolving density distribution, lifetime does not strongly effect the filling fraction.

nosity dependent bias here, nor do we distinguish between the bias of radio-loud and radio-quiet QSOs despite evidence that radio-loud sources are more strongly clustered. Therefore, our bias parameter, $\alpha$, should be considered as an average bias of the outflow-producing population of AGN.

Our fiducial model was run with a constant bias, $\alpha=2$, consistent with the average of many bias models. Because bias increases with redshift, we also ran a model with an increased constant bias of $\alpha=3$. With a larger bias, AGN will be more tightly clustered to higher density regions, preventing their outflows from growing as large, and resulting in more overlap between bubbles. The result is a lower filling fraction, as shown in Figure 2.10.

Since the value of the bias parameter is not constant, but more likely to be (at the very least) redshift dependent, we have also tested the following redshift 
dependent model from Croom et al. (2005):

$$
\alpha(z)=0.53+0.289(1+z)^{2}
$$

The above is a simple model derived from $2 \mathrm{dF}$ data (up to $z<2.48$ ) combined with WMAP and 2dF cosmology. Rather than extrapolate this model over our entire redshift range, for $z \geq 3$ we use a constant bias of $\alpha=5.154$, determined by evaluating the above equation at $z=3$. The effects of redshift dependent bias are seen in Figure 2.10. At higher redshifts, AGN are more strongly biased toward high density regions than in either of our constant bias models, and so the filling fraction is significantly lower. At lower redshifts $(z \lesssim 1)$, AGN are even less biased toward regions of high density than in our fiducial model, and so $F(z)$ increases approaching $z=0$.

\subsection{Discussion and Conclusions}

We have examined the filling fraction of AGN outflows in the context of large-scale cosmological simulations, and considered the influence of various observationally constrained parameters on the result. We find that the kinetic fraction of outflows need not be very high $(\sim 10 \%)$ for AGN outflows to fill the entire IGM by $z \sim 2$. Observations of gaps in the Ly $\alpha$ forest provide possible constraints on the filling fraction, that can in turn be used to place constraints on the kinetic luminosity. In our study we have used a luminosity function consistent with optical surveys to distribute outflows throughout our simulation, however, future studies will have to consider X-ray surveys, which predict more faint luminosity AGN.

Our model employs several simple approximations, but is nonetheless instructive. We have made the assumption that AGN outflows are spherical bubbles,

propagating into the AGN adiabatically for a short while, until reaching pressure 


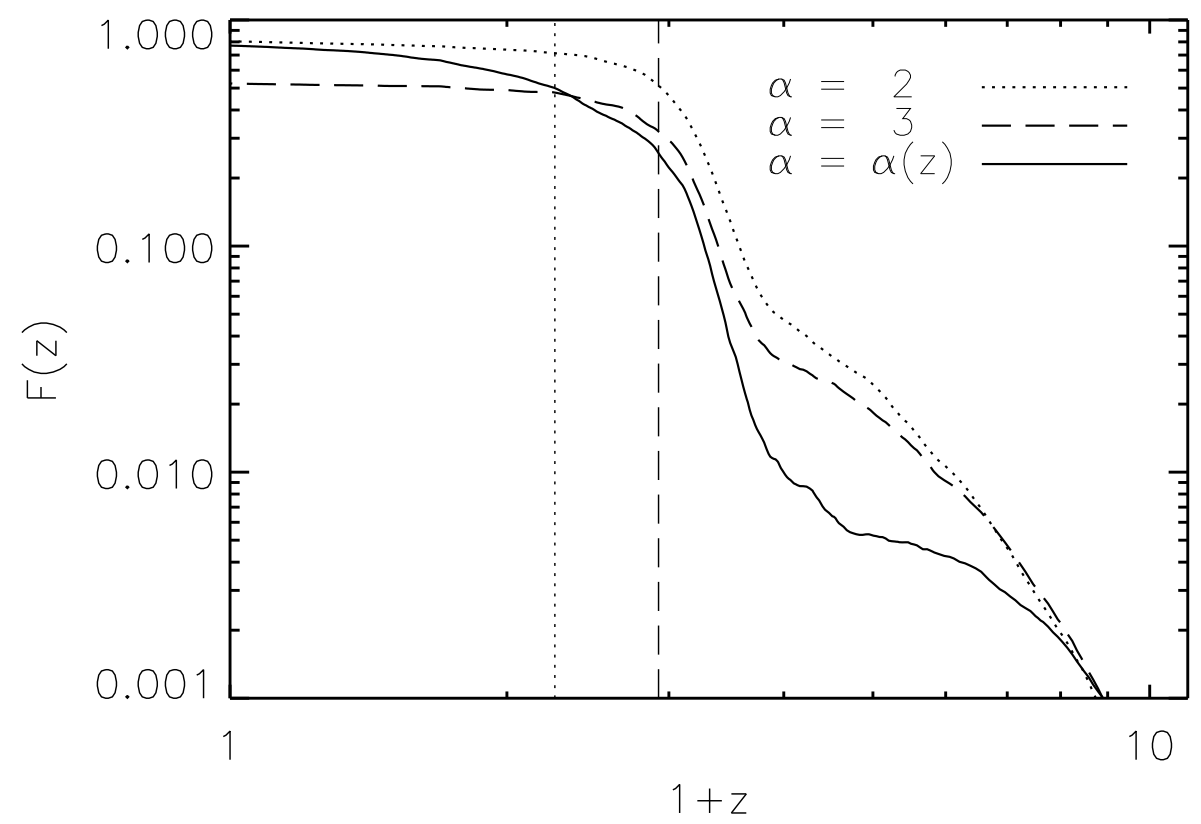

Figure 2.10 Comparison of filling fractions computed with different bias parameters. The two constant bias parameters are shown as well as a z-dependent bias described in $§ 2.4 .3$. The two vertical lines are correspond to the redshifts at which the functional form of the bias equals each of the constant biases (dashed: $\alpha=3$ and dotted: $\alpha=2$ )

equilibrium with their environments. Spherical symmetry is not likely to remain intact once the outflow reaches far enough into the IGM. The outflows will expand away from large-scale structures, such as filaments, and into less dense regions. However, we can assume spherical symmetry as an average geometry, and the effects on the volume filling fraction are not likely to be very large. Our assumption that radiative cooling can be ignored is justified by the timescales involved. If we were to consider more complex environments in our model, such as those of clusters, we would need to include a great deal more physics (including cooling) requiring hydrodynamical simulations. While the actual outflow physics and geometry are likely to be more complex than we assume, our assumptions provide a good first-order approximation of the filling fraction of AGN. 
The parameters we have studied, AGN lifetime, and AGN bias, contribute significantly to the AGN filling fraction as expected. We have assumed a particular evolution of the gas density distribution, in which the gas density profile is relatively uniform at high-z, and forms high density filaments at low-z. We have tested upper and lower limits for AGN lifetime, and find that shorter AGN lifetimes result in a lower filling fraction than longer lived AGN due to the evolving gas density distribution. We have examined the effects of different AGN biases on filling fraction as well. Larger bias results in an ultimately lower filling fraction. However, bias likely depends on factors such as redshift and luminosity, and the dependence of the evolution of the filling factor on bias is likely to be more complicated than in our simple scenario. Changes both in the bias, and in the AGN lifetime affect the filling fraction of outflows because of the importance of the underlying density distribution and its importance for determining AGN distribution and environments. 


\section{Chapter 3}

\section{AGN OUTFLOWS AND THE MATTER POWER SPECTRUM}

Here we investigate the effect of AGN outflows on the matter power spectrum. We continue with the simple model introduced in Chapter 2 and compare the influence of outflows of different kinetic energies on the matter distribution. In $\S 3.1$ we describe the outflow model, and our model of the matter distribution and the corresponding power spectrum. In $\S 3.2$, we show the effects on the power spectrum for different simulation box sizes, resolutions, and outflow energies. Section 3.3 is a summary of our findings and their implications.

\subsection{Simulation}

In the following subsections, we briefly overview a simple model of AGN outflows and the effect they may have on the cosmic density distribution.

\subsubsection{A Simple Outflow Model}

Using a particle-mesh code, we simulate an evolving dark matter distribution under the assumption that the gas distribution follows that of the dark matter. We determine the distribution of AGNs within the simulation by introducing a simple constant bias that assumes AGNs will lie in high density regions. By combining a quasar luminosity function constrained by observations (Cristiani et al., 2004; Fan et al., 2001a,b; Schirber \& Bullock, 2003; Boyle et al., 2000) with 
some simple arguments about the fraction of AGNs expected to host outflows at any given epoch, we obtain the number of sources to include in the simulation.

We assume that following a brief energy injection from the AGN, spherically symmetric outflows expand according to the Sedov-Taylor blast wave model until reaching pressure equilibrium with their environments. They then remain in pressure equilibrium, and any subsequent expansion (or contraction) is due to the Hubble expansion and the evolution of the cosmic density distribution within their neighborhoods. The kinetic energy of the outflow is assumed to be a fixed fraction, $\varepsilon_{k}$ of the AGN's bolometric luminosity. We will investigate the role of different $\varepsilon_{k}$ on the large-scale matter distribution.

In the above outflow model, AGNs fill large spherical bubbles with hot, tenuous gas, pushing aside the gas in the IGM in the process. The intergalactic gas is subsequently compressed into thin shells around the outflows. In our calculation

of the power spectrum, we determine the total matter density distribution by including these thin shells of dense matter into our model.

\subsubsection{Determining the Matter Power Spectrum}

We define the matter density in each cell of the simulation box according to:

$$
\begin{aligned}
1+\delta_{m} & =\frac{\Omega_{d m}}{\Omega_{m}}\left(1+\delta_{d m}\right)+\frac{f_{b} \Omega_{b}}{\Omega_{m}}\left(1+\delta_{d m}\right) \\
& =\left(1+\delta_{d m}\right) \frac{\left(\Omega_{d m}+f_{b} \Omega_{b}\right)}{\Omega_{m}}
\end{aligned}
$$

where $f_{b}$ is a parameter that determines the local baryon fraction. We assume that $\Omega_{d m}=\Omega_{m}-\Omega_{b}$, with 0.27 and 0.04 for $\Omega_{m}$ and $\Omega_{b}$, respectively. Using the dark matter and outflow distributions, we calculate the total matter density in 
the entire simulation volume. We assume that cells lying within outflow regions are basically devoid of baryons, and have $f_{b}=0$. In cells untouched by outflows, we assume the baryon distribution directly traces that of the dark matter and that $f_{b}=1$. At the outflow boundaries, $f_{b}$ is constrained by the average baryon density. We divide the boundary cells into two types: inner and outer boundaries. The weighting parameter for the inner boundary is manually set to lie between 0 and 1 , and the weighting parameter for the outer boundary is then determined by the normalization. The results do not appear to have a significant dependence on our choice of $f_{b}$ at the boundaries. The values adopted for $f_{b}$ in the present model are an over-simplification, and will provide an upper-limit for the effects of AGN outflows on the matter power spectrum.

We calculate the matter power spectrum for the case in which baryons closely follow the dark matter distribution (without AGN outflows) and for the case in which baryons are redistributed by outflows. Specifically we are interested in the quantity

$$
\Delta P(k)_{\mathrm{AGN}}=\frac{P(k)_{\mathrm{AGN}}}{P(k)_{\mathrm{nobar}}}-1,
$$

where $P(k)_{\text {AGN }}$ is the power spectrum accounting for AGN outflows and $P(k)_{\text {nobar }}$ is the power spectrum determined from dark matter alone (with baryons tracing dark matter at our resolution).

The outflows affect the large scale power via two competing effects in this model. First, outflows move gas around, redistributing baryons from high to low density regions. This has the effect of decreasing the matter power on a range of scales. Second, if AGN outflows only affect the small-scale, immediate environment of host galaxies, high clustering of AGNs transfers the fluctuations from small to large scales. This can be thought of symbolically ${ }^{1}$ by representing

\footnotetext{
${ }^{1}$ Equations 3.4 and 3.5 are not equations in the strict sense, but rather symbolic representations used to concisely convey the physical ideas.
} 
the gas density as

$$
\rho_{g}(\mathbf{x})=\hat{L S}\left[\rho_{g, 0}(\mathbf{x})\right]+\hat{S S}\left[n_{A G N}(\mathbf{x})\right]
$$

where the gas density $\rho_{g}(\mathbf{x})$ is represented as the sum of the Large-Scale (LS) redistribution of the "undisturbed" gas density $\rho_{g, 0}$ and additional Small-Scale (SS) density fluctuations caused by complex gas dynamical motions around AGNs. The quantities $\hat{L S}$ and $\hat{S S}$ are operators in the strict mathematical sense, but if we approximate them as convolutions with some window functions, then symbolically, the baryonic power spectrum is

$$
P_{g}(k)=W_{L S}^{2} P_{g, 0}(k)+W_{S S}^{2} P_{A G N}(k)+\text { Cross Terms }
$$

where the Large-Scale factor $W_{L S}^{2}$ is, generally smaller than 1 , since one would expect the AGN outflows to move gas from high to low densities, thus reducing the clustering of gas (although, the AGN effect may be very small, in which case $W_{L S}^{2}$ could be indistinguishable from unity). The Small-Scale factor $W_{S S}^{2}$ is likely to be small, since only a small fraction of all galaxies host AGNs at any given moment, but it is multiplied by a large factor $P_{A G N}(k)$ - the latter is large since AGNs are highly clustered, with bias factors as high as 5, depending on redshift and luminosity (e.g. Croom et al., 2005; Lidz et al., 2006). In our model, $W_{S S}^{2}$ represents spherically symmetric bubbles, which behave similarly for all AGNs in the simulation. In reality, it should be a much more complex factor, depending on the relevant physics and the AGN environment on smaller scales. The contribution of the second term in Equation 3.5 also depends on the amount of bias in the AGN distribution. A high bias factor can increase the size of the AGN term significantly. The important point to take is that the AGN term need not be very large $(10 \%)$ to change the amplitude of the power spectrum by an amount sufficient to bias 
lensing measurements $(1 \%)$.

\subsection{Results}

In $§ 3.2 .1$ and $§ 3.2 .2$, we conduct convergence studies of simulation box size and resolution and we study the effects of varying the kinetic fraction, $\varepsilon_{k}$, on the amplitude of the matter power spectrum. We show the following power spectrum results for $z=0.81$, corresponding to the mean redshift of sources in the first analysis of the Canada-France-Hawaii Telescope Survey (Hoekstra et al., 2005), which used weak lensing to make predictions for cosmological parameters.

\subsubsection{Convergence Studies}

To test the effects of resolution on the matter power spectrum results, we have calculated the power spectrum for simulation boxes of length $64 h^{-1} \mathrm{Mpc}$ with resolutions of $1,0.5$, and $0.25 h^{-1} \mathrm{Mpc}$. Figure 3.1 shows the resulting $\Delta P(k)_{\mathrm{AGN}}$ at two different redshifts. At higher- $z$, the results are somewhat similar for simulations of different resolutions, showing a negative contribution to the amplitude of the matter power spectrum. At $z=0$, the two higher resolution runs both show positive contributions to the power spectrum. In the high- $z$ case, the AGN outflows move gas around, effectively lowering the amplitude of the power spectrum, while at $z=0$ it is possible that the effects of AGN clustering dominate, at least for the higher resolution runs.

Figure 3.2 shows a comparison of the results for boxes of different lengths, but each with the same resolution of $1 h^{-1} \mathrm{Mpc}$. The box lengths shown are 64, 128, and $256 h^{-1} \mathrm{Mpc}$. The results for each box size are fairly similar, each showing a negative contribution of $\sim 20 \%$ to the amplitude of the power spectrum.

Figure 3.3 shows the redshift evolution of $\Delta P(k)_{\text {AGN }}$ for boxes of length 64 and $128 h^{-1} \mathrm{Mpc}$, with $0.5 h^{-1} \mathrm{Mpc}$ resolution, as well as a $64 h^{-1} \mathrm{Mpc}$ box with 


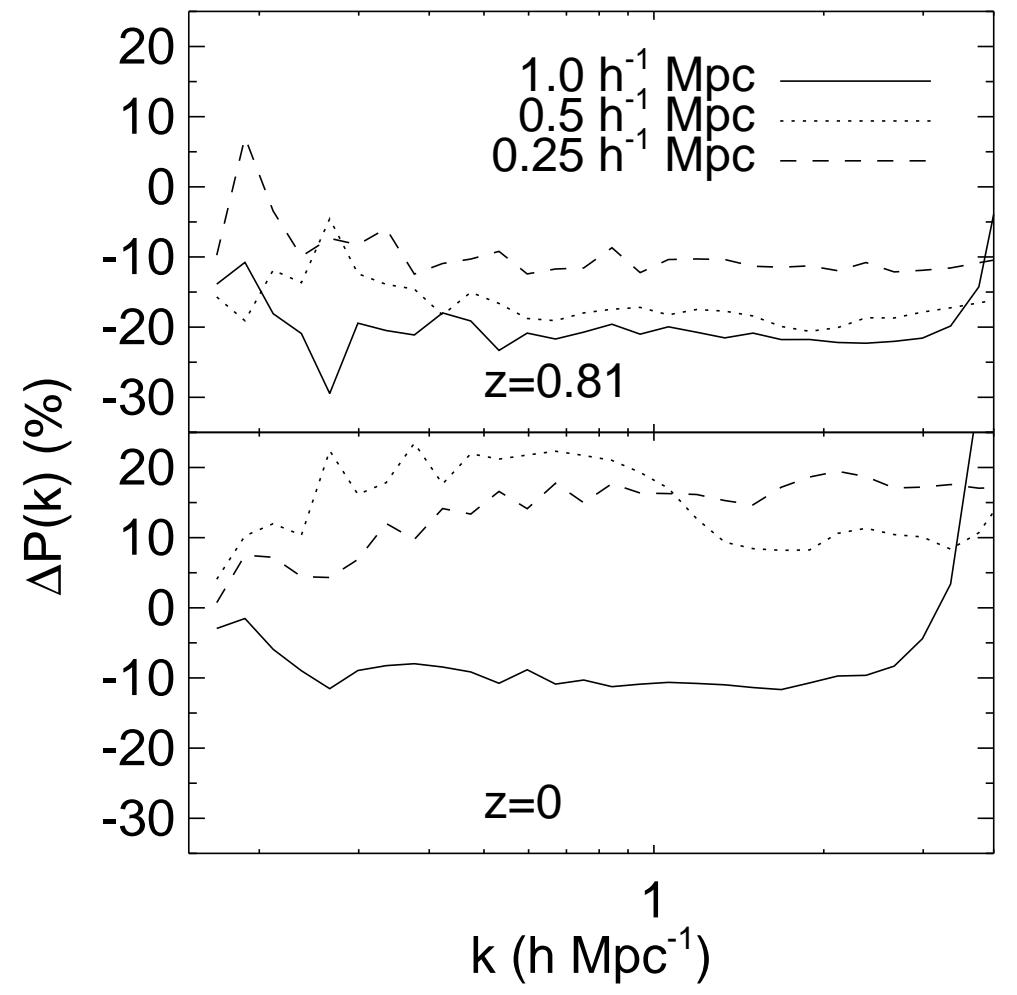

Figure 3.1 Percentage difference between the matter power spectrum with and without the AGN outflow model for simulations of differing resolutions at two different redshifts. Each box is $64 h^{-1} \mathrm{Mpc}$ across.

a higher bias factor $b$. For $z>1, \Delta P(k)_{\text {AGN }}$ decreases as the outflows expand, moving gas onto larger scales. As the filling fraction of AGN outflows levels off at low- $z$, the positive contribution of AGN clustering begins to take over, causing the turnaround in $\Delta P(k)_{\mathrm{AGN}}$ shown for the smaller box. At low- $z$, the larger volume simulation contains more bright AGNs (larger bubbles) than the smaller volume, so the negative contribution of AGN outflows to the power spectrum continues to dominate. The run with the higher bias factor also demonstrates the positive contribution of AGN clustering to $\Delta P(k)_{\mathrm{AGN}}$, since $\Delta P(k)_{\mathrm{AGN}}$ is greater for $b=3$ than for $b=2$.

The convergence studies support our interpretation of the Large-Scale redistribution term in Equation 3.5, because in the case where the negative contri- 


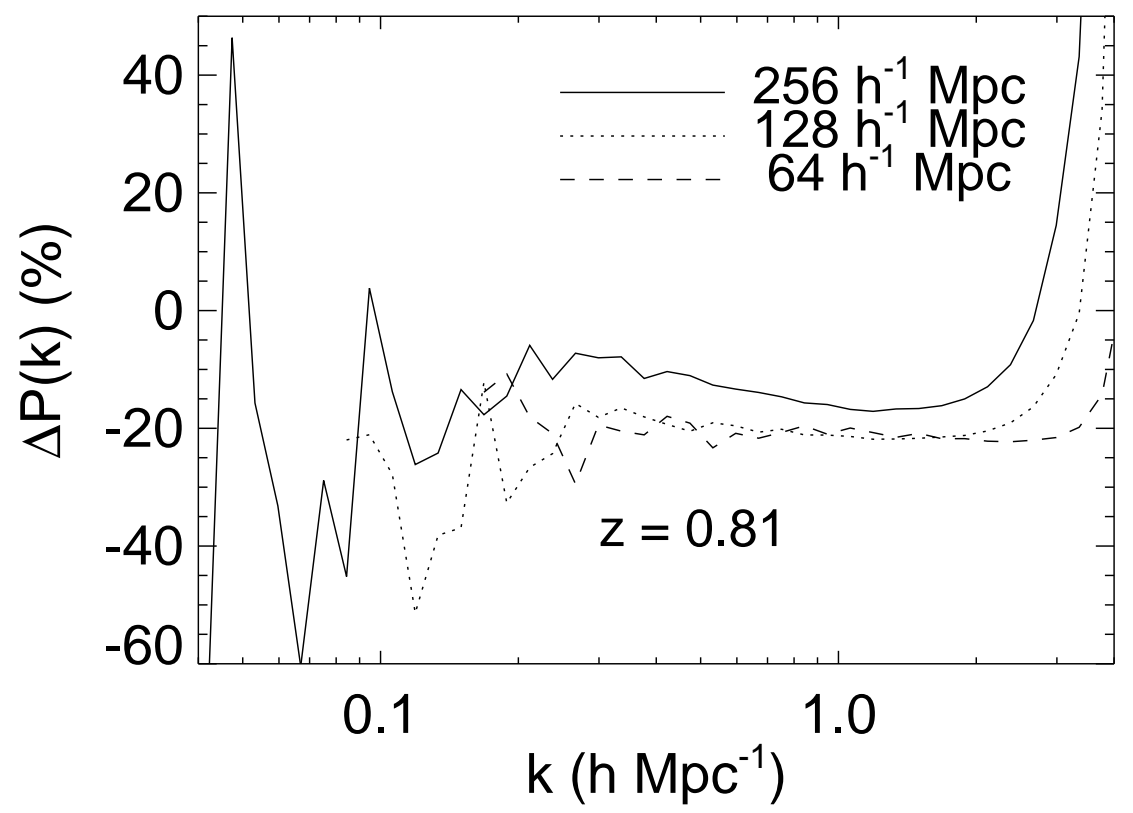

Figure 3.2 Percentage difference between the matter power spectrum with and without the AGN outflow model for different simulations volumes. Each box has cells of length $1 h^{-1} \mathrm{Mpc}$.

bution of outflows dominates (in the low-resolution simulations; see Figure 3.2), some convergence takes place. In the resolution study convergence is not as obvious, as is demonstrated in Figures 3.1 and 3.3. The second term in Equation 3.5 implies that it is difficult to reach convergence without detailed modeling of the small-scale fluctuations.

\subsubsection{Dependence of the Matter Power Spectrum on Kinetic Frac- tion}

The kinetic energy driving outflows in AGNs is likely linked to the luminosity of the AGNs, but the exact fraction, $\varepsilon_{k}$ is not yet well constrained by observations. As studies of the filling fraction of AGN outflows showed in Chapter 2, the more kinetic energy input from the quasar, the greater the effect on 


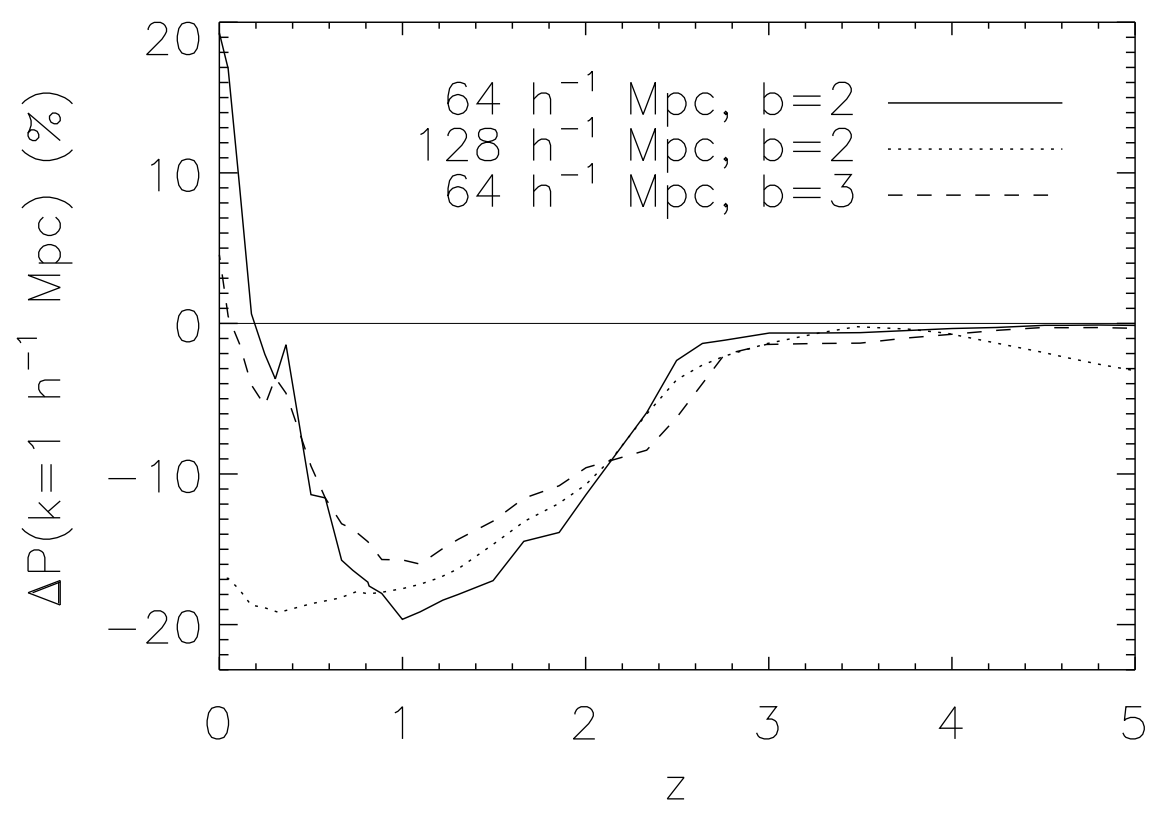

Figure 3.3 Redshift evolution of the percentage difference between the power with and without the AGN outflow model at $k=1 h \mathrm{Mpc}^{-1}$ for $0.5 h^{-1} \mathrm{Mpc}$ resolution boxes. Two different box sizes and two different bias factors are shown. The turnaround in $\Delta P(k)_{\mathrm{AGN}}$ could be a result of AGN clustering effects dominating over the redistribution of gas by outflows.

the AGN environments. In this simple outflow model, it only takes a very small fraction of the energy output of AGNs to produce outflows that fill the entire IGM by $z=2$. In our convergence studies, we have adopted a $\varepsilon_{k}$ of $1 \%$. Here we examine the effects of varying $\varepsilon_{k}$ on the power spectrum. Figure 3.4 shows that as little as $1 \%$ of an AGN's bolometric energy output produces changes to the power spectrum of up to $20 \%$ in the simple model presented here. A $\varepsilon_{k}$ of $2 \%$, or a doubling in the efficiency of the AGN, does not drastically change the effects on the matter power spectrum. The precise dependence of the amplitude of the power spectrum on the kinetic fraction likely depends on the properties of AGNs in a non-trivial way. 


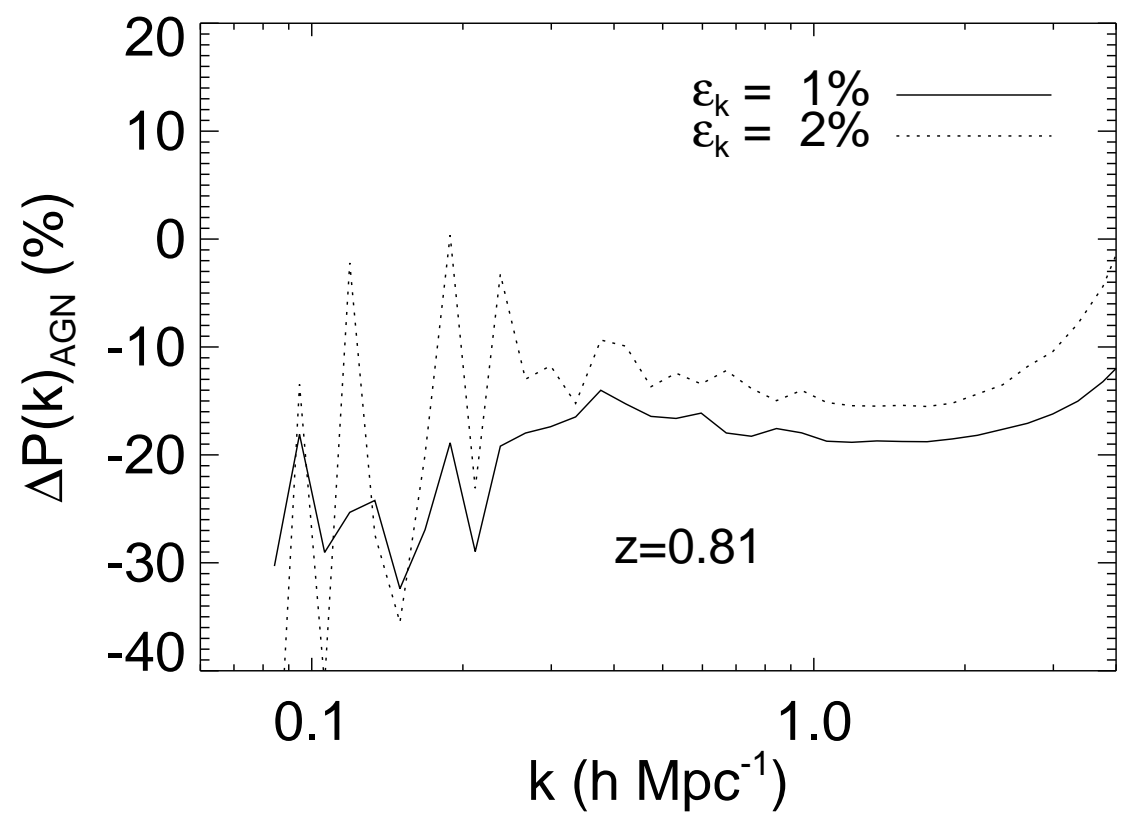

Figure 3.4 Percentage difference between the matter power spectrum with and without the AGN outflow model for models with different kinetic fractions, $\varepsilon_{k}$.

\subsection{Discussion and Conclusions}

We have found that a simple model in which AGN outflows influence the distribution of baryonic matter on cosmic scales results in more than several percent difference in the amplitude of the matter power spectrum. Two competing effects - the removal of gas from high density regions and high clustering of AGNs - make contributions of opposite signs to the matter power.

The amount of energy released in observed AGNs may be sufficient to move all of baryons in the universe over cosmological distances, which would result in the reduction in the matter power spectrum by up to $30 \%$. Observationally, we do not really know whether AGNs do that or not, but they definitely have the means. It is also possible that the AGN outflows get stopped in the central parts of galaxies, never reaching cosmological scales, or that, even if they reach cosmological scales, 
outflows expand more quickly into low density regions without affecting higher density regions much.

Additionally, AGNs clustering might influence the power on large scales. Even if AGNs cause non-gravitational fluctuations only on smaller, sub-Mpc scales, these fluctuations propagate to larger, tens of Mpc scales, because the AGNs themselves are clustered more than the baryons as a whole.

Admittedly, our model of spherical outflows is excessively simplistic, and should probably only be considered as an upper limit. Nevertheless, it presents a counterexample to the widespread belief that lensing measurements are insensitive to complex astrophysics.

Unfortunately, the interplay between the two competing effects of AGNs is quite intricate, so even the sign of the total effect of AGN outflows on the matter power spectrum cannot be deduced without further detailed numerical studies of complex gas dynamics on galactic and sub-galactic scales. But the final success of future weak lensing studies of the dark energy will substantially depend on our ability to make a theoretical breakthrough in modeling AGN outflows on a wide range of scales. 


\section{Chapter 4}

\section{FROM MEGAPARSECS TO MILLIPARSECS: A COSMOLOGICAL SIMULATION MODELING BLACK HOLE GROWTH}

\subsection{Introduction and Motivation for doing the simulations}

As the first three chapters have suggested, understanding the fueling of SMBHs and the potential impact they have on their environments requires sophisticated numerical techniques, capable of following complicated hydrodynamical processes and astrophysical phenomena (such as star formation and feedback, heating and cooling of gas, etc.). However, it is unfeasible to follow the evolution of a galaxy all the way down to the scale of a black hole accretion disk in such detail over cosmological time. Such a simulation spans a large range of scales (with

a dynamic range $>10^{7}$ ), and each spatial scale has a different temporal scale. As a result, the Courant condition for numerical stability requires very small time steps in the most highly refined regions, making it computationally expensive to follow the evolution of the galaxy over long periods of time. It is much more feasible to start with a lower resolution cosmological simulation and zoom in to the small-scale region with increasingly smaller cell sizes and time steps. As the simulation evolves on small scales, the large-scale portion of the simulation does not undergo much evolution and does not need as high resolution.

The numerical technique of Adaptive Mesh Refinement (AMR) is ideal for astrophysical problems spanning a large dynamic range, as demonstrated by sim- 
ulations of the formation of the first star (Abel et al., 2002), the collapse of protogalactic halos (Wise, 2007), and turbulent star formation in the interstellar medium (e.g. Truelove et al., 1998; Krumholz et al., 2007). The technique allows a simulation to follow each scale with the appropriate resolution for modeling the relevant physics, without wasting computational power on unnecessary resolution elsewhere. AMR is therefore well suited for studying the growth of SMBHs in a cosmological context.

\subsection{ART}

The cosmological simulations employed here use the Adaptive Refinement Tree (ART) code (Kravtsov et al., 1997; Kravtsov, 1999; Kravtsov et al., 2002). The ART code uses the AMR technique, allowing high resolution of a galaxy residing in a small region of a cosmological simulation while following the rest of the simulated volume with lower resolution. The technique is appropriate for studying the structure and evolution of a galaxy over a large dynamical range and in a cosmological context.

The ART code includes a range of physics for modeling dark matter, stars, and gas dynamics, most of which is included in the present simulations. Gas is converted into stars in cells with densities greater than $\rho_{\mathrm{SF}}$ and temperatures less than $T_{\mathrm{SF}}$, where $\rho_{\mathrm{SF}}=50 \mathrm{~cm}^{-3}$ and $T_{\mathrm{SF}}=9000 \mathrm{~K}$ (see Kravtsov, 2003, for more details), resulting in a star formation efficiency consistent with a Kennicutt law on kiloparsec scales (Kennicutt, 1998) and with observations on 100pc scales as well (e.g. Young et al., 1996; Wong \& Blitz, 2002). The code follows ISM physics, such as molecular hydrogen formation and gas cooling by heavy elements and dust under the assumption of collisional ionization equilibrium. The cooling and heating rates are tabulated as functions of gas density, temperature, metallicity, and redshift over the temperature range $10^{2}<T<10^{9} \mathrm{~K}$ using CLOUDY (Ferland 
et al., 1998), which accounts for the metallicity of the gas and formation of molecular hydrogen and cosmic dust. In future studies, we plan to include the ART code's radiative transfer capabilities, which will be necessary for implementing AGN feedback.

\section{3 "Zooming In" to the Center of a Galaxy}

We begin with a cosmological simulation, evolved from a realization of a random Gaussian density field at $z=50$, with periodic boundary conditions, measuring $6 h^{-1}$ comoving Mpc across. The simulation was first run with low resolution in order to select a galactic mass halo for subsequent study. A Lagrangian region the size of 5 virial radii of the largest halo at $z=0$ was then identified at $z=50$ and re-sampled and run with higher resolution to $z=2.8$ (see Klypin et al., 2001, for a description of the technique). The Lagrangian region of the simulation was automatically refined three levels, as a minimum. There are $2.64 \times 10^{6}$ dark matter particles in the Lagrangian region, each with mass $9.18 \times 10^{5} h^{-1} M_{\odot}$ : 64 times less massive than those at large distance. Subsequent refinement and derefinement in the Lagrangian region of the cosmological simulation followed a dark matter mass criterion, in which a cell was refined if its total dark matter mass was greater than $1.8 \times 10^{6} h^{-1} M_{\odot}$. Each time a parent cell in the simulation "refines," it splits into 8 child cells, doubling the spatial resolution in that region.

At $z=4$, the highest matter density peaks in the simulation have a maximum resolution of $\approx 50 \mathrm{pc}$ (in physical units; corresponding to nine levels of refinement on top of a $64^{3}$ root grid). The largest halo has a total mass of $2 \times 10^{11} M_{\odot}$ at $z=4$, and it contains the progenitor of an $L^{*}$ spiral galaxy. This initial cosmological simulation was run including metal enrichment and energy feedback from supernovae, and radiative transfer, in addition to the physics described in $§ 4.2$. The stellar component of the galaxy is shown in Figure 4.1, in both a face-on and 


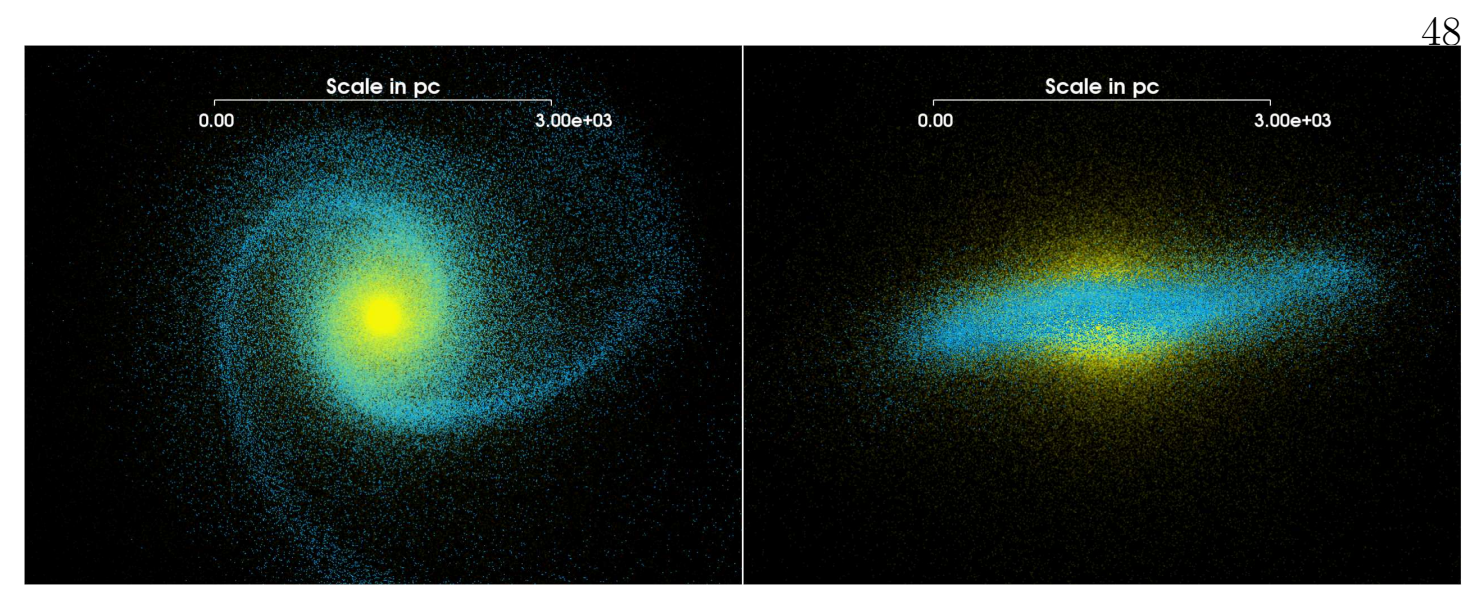

Figure 4.1 Stellar component of the galaxy at $z=4$ on a scale of $3 \mathrm{kpc}$. The stars in the image are divided into two populations, with yellow stars corresponding to the oldest stars in the galaxy and blue stars corresponding to the youngest stars. The youngest stars are found primarily in the disk of the galaxy. This figure is best viewed in color.

an edge-on view on a scale of 3 physical kpc. The two different colors correspond to old and young stars (yellow and blue, respectively), with the youngest stars in the galaxy located primarily in the disk and the oldest stars distributed more isotropically, populating the galaxy's bulge.

The highest resolution portion of the simulation begins by allowing further refinement inside the cosmological simulation, one level at a time, in a $1.5 \mathrm{kpc}$ region centered on the galaxy, effectively zooming in to the center of the galaxy with increasing resolution. The "zoom-in" technique is similar to the one used by Abel et al. (2002) in simulations of the formation of the first star, which required an even larger dynamical range. In the first set of simulations (this thesis) we are starting simply, and planning to build a more realistic simulation in parts. Therefore, radiative transfer and stellar feedback have been switched off in the zoom-in portion of the cosmological simulation (they will be examined in future studies). In the high density regions of the simulation, the assumption of ionization equilibrium produces results similar to those of simulations that include radiative transfer (see Tassis et al., 2008). Therefore the absence of radiative transfer in 
the "zoom-in" portion of our simulations does not significantly affect the results.

The high-resolution portion of the simulation employs additional refinement criteria, refining according to a level-dependent mass criterion on levels 11 and below $^{1}$ in the zoom-in region. The refinement criterion is defined so that the finer the resolution, the more aggressively the mesh refines, ensuring that there are enough highly refined cells to resolve structures on small scales. Specifically, the mesh refinement is super-Lagrangian in the circumnuclear region of the galaxy, and cells are marked for refinement if the gas mass in a cell is $m_{\mathrm{r}}^{\text {level-10 }}$ times the Lagrangian mass criterion $\left(9 \times 10^{7} h^{-1} M_{\odot}\right)$, where $m_{\mathrm{r}}=0.7$ is our fiducial value. The factor 0.7 was chosen through experimentation, in order to populate the central region with enough high-resolution cells to resolve structures, but not to produce so many cells that the simulation becomes too computationally expensive.

During the zoom-in period, we increase the maximum level of refinement gradually, one level at a time, allowing the simulation to reach a quasi-stationary state on each level before moving to the next. The slow initial refinement allows the ART code to resolve spatial scales within the simulated galaxy while avoiding transient effects. Since the time steps depend on the sound speed of the gas, they are related to the dynamical time, and a fixed minimum number of time steps on each level forces the mesh to evolve for several dynamical times. We have run parallel simulations with 300 and 1000 minimum steps on each level (runs C120B and $\mathrm{C} 120 \mathrm{C}$, respectively) and determined that a requirement of a minimum of 300 on each level sufficiently reduces transient numerical effects that result from refining too quickly. A third run stepping 100 times on each level during the initial refinement continued to show transient behavior well after reaching the maximum level of refinement, and was abandoned for this reason.

\footnotetext{
${ }^{1}$ We adopt the convention of referring to level 0 as the "top" level and the maximum (most refined) level as the "bottom" level, so that the terms "above" and "below" refer to lower and higher resolution, respectively.
} 


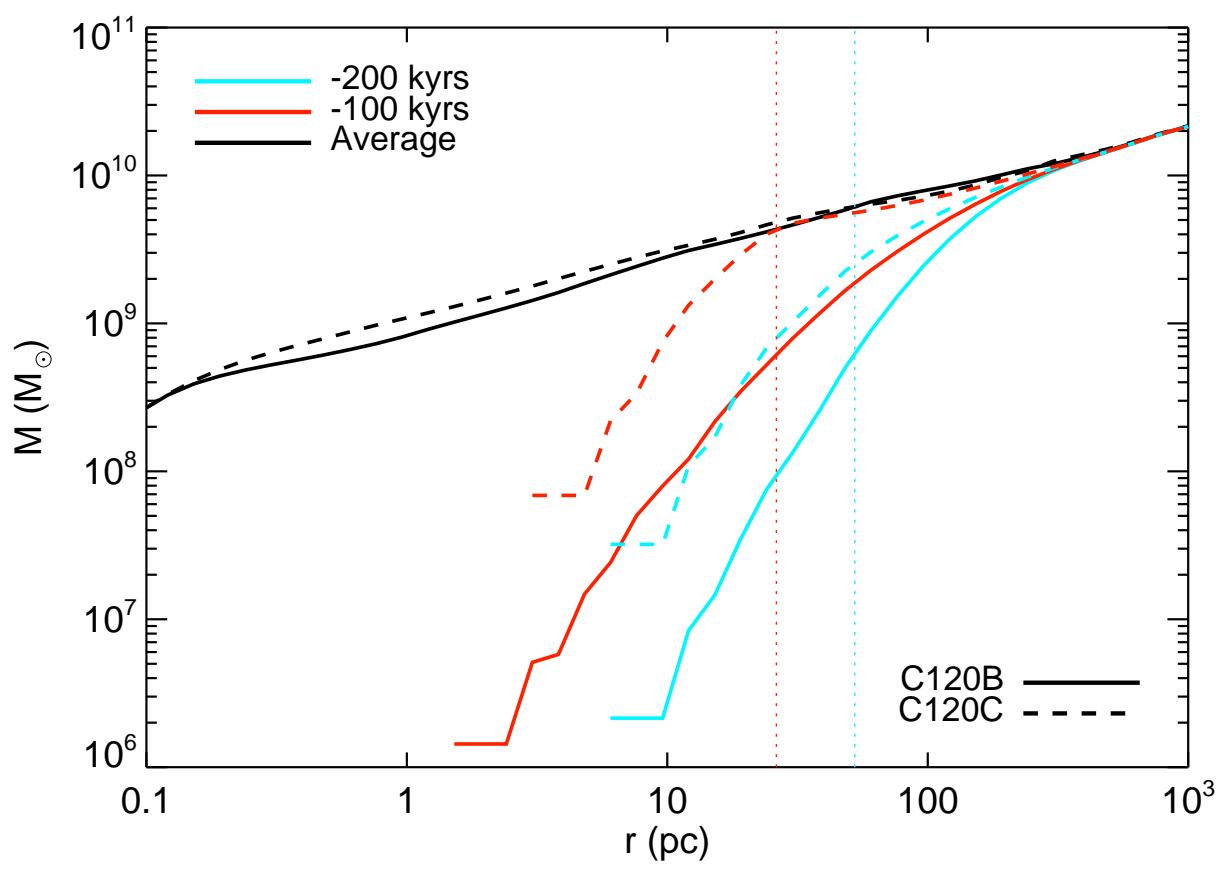

Figure 4.2 Interior gas mass in the central kiloparsec for runs C120B and C120C (solid and dashed curves, respectively). The black line gives a time-average taken after reaching 20 levels of refinement, and the colors represent individual snapshot during the initial zoom-in. The vertical dotted lines give the approximate resolution limit at the corresponding times. The flattening of the color curves at small $r$ is a result of cell discreteness.

Figure 4.2 shows the interior gas mass in the central kiloparsec of the galaxy for runs C120B (solid curve) and C120C (dashed curve). The figure shows timeaverages of the interior gas mass (black curves) taken after reaching level 20, in addition to profiles at specific times during the initial zoom-in episode. The vertical dotted lines give the approximate resolution limit during zoom-in, given by 4 maximum level cells at the corresponding times. The mass profile approximates the simulation average more closely in the case of slower initial refinement, especially on scales which are well resolved.

After the initial zoom-in, the highest level cells are $0.03 \mathrm{pc}$ across (corresponding to 20 levels of refinement), allowing us to determine the location for a SMBH particle with high precision but without resolving the black hole accre- 
tion disk, which would require additional physics (such as magnetohydrodynamics [MHD]) not currently included in the ART code. MHD is necessary for modeling accretion disk physics because magnetorotational instabilities drive the turbulence which allows gas transport through the accretion disk (Hawley et al., 1995). Therefore, the simulations here follow the relevant physics reliably down to a scale of at least $0.12 \mathrm{pc}$ (outside the accretion disk), or the size of four level 20 cells, which we consider to be our resolution limit.

It is necessary to refine slowly, all the way to the maximum resolution before inserting a black hole particle, or else transient structures arising during the zoomin process will displace the black hole from the center of mass of the galaxy. The mass of the black hole in the fiducial run at $z=4$ is $3 \times 10^{7} M_{\odot}$. Upon reaching the maximum resolution (level 20), we replace a fraction of the gas from the center of the simulated galaxy with a black hole point mass of equal mass and momentum. Mass is only drawn from cells above a given gas density threshold, determined by requiring that the mass is collected from within the circumnuclear region (the central $\left.300 h^{-1} \mathrm{pc}\right)$. Each contributing cell donates a fraction of its mass,

$$
\frac{\Delta M_{\text {cell }}}{M_{\text {cell }}}=1-\frac{\rho_{\text {th }} V_{\text {cell }}}{M_{\text {cell }}},
$$

where $\rho_{\text {th }}$ is the density threshold. There is typically enough gas mass contributed from within the central parsec to create a $3 \times 10^{7} M_{\odot}$ black hole, as is shown in Figure 4.3. The figure 4.3 shows the fraction of the black hole mass contributed by individual cells as a function of radius for the runs C120B and C120C.

At present, we focus on the dynamical properties of the circumnuclear disk on scales where the gravity of the gas dominates that of the black hole. The addition of the black hole point mass here simply allows us to follow the black hole's location and velocity as a reference point. However, the successful introduction of 




Figure 4.3 The fraction of the black hole particle mass contributed by individual cells as a function of radius in run C120C (grey circles) and run C120B (diamonds). During the slower zoom-in episode (C120C), black hole mass is only drawn from level 20 (filled diamonds), whereas the faster zoom-in (C120B) draws black hole mass from level 19 cells (open diamonds) as well.

the black hole particle will be essential in subsequent simulations involving physics associated with the black hole (such as AGN feedback).

After the initial zoom-in and the successful introduction of the black hole particle, the simulation then evolves at high resolution for approximately one dynamical time at the 100pc scale, showing a highly resolved galactic disk. For a Keplerian disk, the number of orbital periods, $N_{\text {orb }}$, undergone at radii less than $100 \mathrm{pc}$ is $(R / 100 \mathrm{pc})^{-3 / 2}$. For the quasi-Keplerian disk in the present simulations, the disk mass is significant and the precise scaling depends on the slope of the density distribution. Figure 4.4 shows the three-dimensional gas density in an approximately face-on view of the galactic disk, on several different spatial scales, 
for a single time step, demonstrating the large dynamic range of the simulation.

\subsection{Numerical Issues}

\subsubsection{A Test of Angular Momentum Conservation}

Anomalous numerical transport of angular momentum is sometimes presented as a concern for adaptive mesh refinement simulations using interpolation schemes for calculating velocities on the mesh. As a preliminary test for the accuracy with which the angular momentum is conserved in the ART code, we have run a test simulation of an isothermal, uniformly rotating sphere. Such an initial setup is immediately unstable to collapse, forming a thin disk, which conserves angular momentum in the absence of any dissipative process (e.g. Mestel, 1963; Crampin \& Hoyle, 1964). Therefore, by comparing the angular momentum before and after the collapse, the setup provides a simple test of angular momentum conservation for the ART code.

Beginning with a $1 \mathrm{Mpc}$ box containing $32^{3}$ top-level cells, the initial setup includes a sphere of radius $r_{\mathrm{s}}=0.1 l_{\mathrm{box}}$, with a boundary of width $\Delta r=0.1 r_{\mathrm{s}}$. The initial sphere is resolved with 2 levels of refinement (corresponding to $\sim 8000 \mathrm{pc}$ ). The temperature of the sphere is $10^{4} \mathrm{~K}$. The density outside the sphere, $\rho_{\mathrm{i}}$ is $2.8 \times 10^{6} M_{\odot} \mathrm{Mpc}^{-3}$, and the density inside, $\rho_{\mathrm{s}}$ is $2.8 \times 10^{11} M_{\odot} \mathrm{Mpc}^{-3}$. For the

collapsing sphere test, cosmological parameters are all set to unity. The sphere also contains a boundary region $\left(r_{\mathrm{s}}-\Delta r<r<r_{\mathrm{s}}+\Delta r\right)$, where the density is given by

$$
\rho(r)=\rho_{\mathrm{i}}+\frac{\rho_{\mathrm{s}}}{1+\exp ^{\left(r-r_{\mathrm{s}}\right) / \Delta r}}
$$

The initial angular velocity of the sphere is 


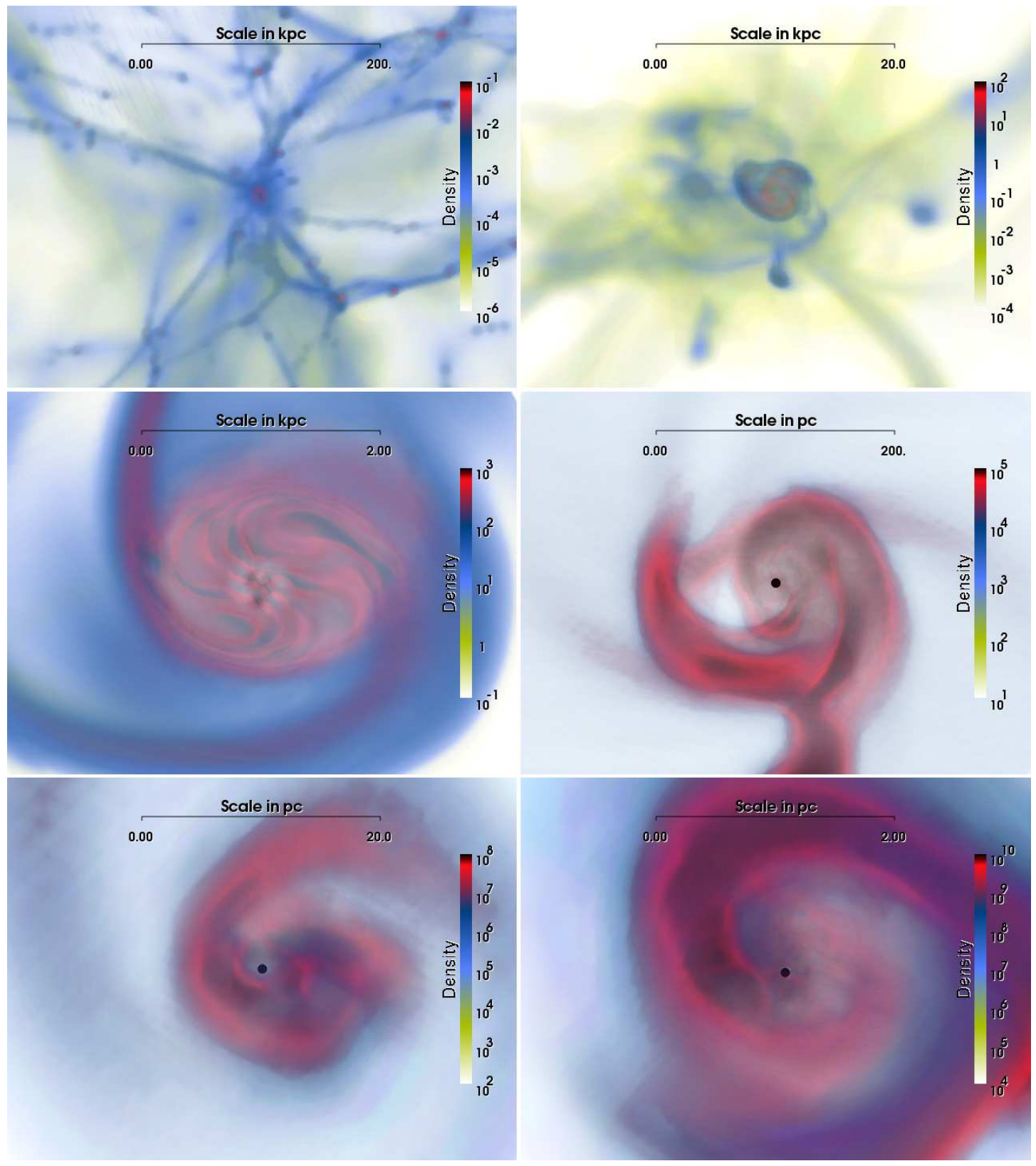

Figure 4.4 Volume rendering of the three-dimensional gas density on several different scales at $z=4$, from $200 \mathrm{kpc}$ in the top left panel to $2 \mathrm{pc}$ in the bottom right panel. The color bar shows the density scale in units of $\mathrm{cm}^{-3}$. Note the presence of the spiral arms and instabilities on a wide range of scales. This figure is best viewed in color. 
Table 4.1 Initial conditions for collapsing sphere test

\begin{tabular}{||l|c||}
\hline Parameter & \\
\hline \hline Box length, $l_{\text {box }}$ & $1 \mathrm{Mpc}(32$ top level cells $)$ \\
\hline Radius of sphere, $r_{\mathrm{s}}$ & $0.1 \mathrm{Mpc}$ \\
\hline Temperature, $T$ & $10^{4} \mathrm{~K}$ \\
\hline Density outside sphere,$\rho_{\mathrm{i}}$ & $2.8 \times 10^{6} M_{\odot} \mathrm{Mpc}^{-3}$ \\
\hline Density inside sphere ${ }^{*} \rho_{\mathrm{s}}$ & $2.8 \times 10^{6} M_{\odot} \mathrm{Mpc}^{-3}\left(10^{5} \rho_{\mathrm{i}}\right)$ \\
\hline
\end{tabular}

*excluding boundary region; given in units of the critical density, $\rho_{\text {crit }}$

$$
\Omega_{\mathrm{s}}=\pi \sqrt{G \rho_{\mathrm{s}} \frac{r_{\mathrm{d}}}{r_{\mathrm{s}}}},
$$

as given by Mestel (1963), where $r_{\mathrm{d}}$ is the radius of the final disk as long as angular momentum is conserved. The initial conditions are summarized in Table 4.4.1.

Following the initial setup, the sphere collapses into a thin disk $\left(h / r_{\mathrm{d}} \ll 1\right.$, where $h$ is the disk thickness), which conserves angular momentum over several rotation periods. Crampin \& Hoyle (1964) characterize the developing mass distribution in terms of the specific angular momentum as

$$
\frac{d M}{d j}=C\left(1+\frac{j}{j_{\max }}\right)^{1 / 2},
$$

where $C$ is a constant, $j$ is the specific angular momentum, and $j_{\max }$ is the maximum specific angular momentum of the initial sphere. The two sets of points in Figure 4.5 show the angular momentum distribution of the initial conditions and the same distribution at the moment when the central density has increased by a factor of $10^{5}$ (after several rotation periods). The distribution, $d M / d j$, gives the mass per specific angular momentum bin, which should be conserved throughout the collapse. The points match the theoretical prediction for the mass distribution given by Equation 4.4 (and shown as the solid curve in Figure 4.5) at each time. 




Figure $4.5 \mathrm{~A}$ test of the angular momentum conservation during a multi-level refinement in the ART code. The filled circles show the angular momentum distribution of a uniformly rotating sphere set on a uniform mesh. The open diamonds are the same distribution when the sphere is allowed to collapse by 8 levels of refinement. The solid line is the theoretical prediction from Crampin \& Hoyle (1964, a small deviation at high values of $J$ is due to mesh discreteness).

\subsubsection{Dark Matter Discreteness Effects}

Another concern regarding numerical artifacts is the discreteness of dark matter particles. Dark matter is modeled in the simulations as collisionless particles that interact by a softened gravitational force. There is no known satisfactory way to split a dark matter particle into several when the mesh is refined. Thus the dark matter particles appear as massive, discrete objects in highly refined parts of the simulation. Fortunately, the places where the simulation becomes most refined are precisely the places where the baryonic density becomes high enough so that its gravity dominates over that of the dark matter. Nonetheless, potential 
numerical artifacts should be avoided.

In the most resolved parts of the simulation, the discreteness of dark matter particles can be eliminated by restricting the dark matter gravity calculations to sufficiently low levels. For example, at the center of our simulated galaxy there may be many cells of level 20 for each dark matter particle. However, if we limit the calculations of the gravitational force of the dark matter to levels 9 and above, where there are several $(\approx 15-20)$ dark matter particles per each cell, the discreteness artifact disappears as the density assignment scheme in the ART code will result in a smooth and continuous distribution of the dark matter over the whole mesh.

We have selected a sample of dark matter particles in the cosmological simulation (with 9 refinement levels) and followed their positions and velocities throughout the higher resolution portion of the simulation. The particles were chosen randomly from those within the central kiloparsec of the galaxy at $z=4$. Figure 4.6 shows the velocity and radial distance of each particle in the sample in the initial cosmological simulation, 100kyr before reaching level 20 and the appearance of the black hole particle (12 levels of refinement), 100kyr after, and 900kyr after. As the initial cosmological simulation refines, the mass distribution in the galaxy becomes more centrally concentrated, so that the dark matter particles located near the center reach higher velocities. However, the range of velocities of dark matter particles located farther from the center should be comparable to that of the initial cosmological simulation. Among the sample of 1,000 particles, very few $(\approx 1 \%)$ show anomalously high velocities after 900kyr, and the few that do appear to have been scattered off of dense structures in the circumnuclear disk. 


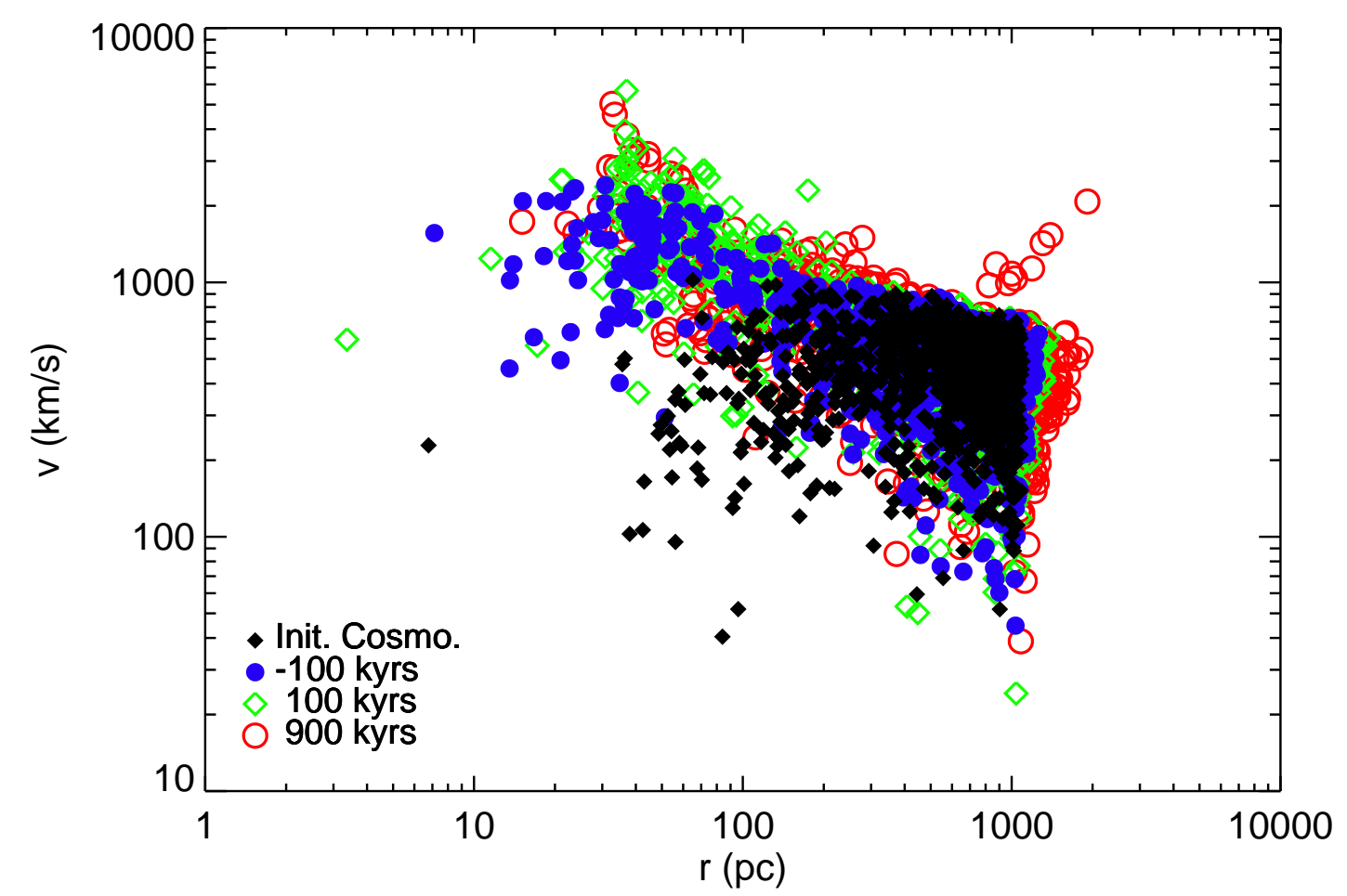

Figure 4.6 Velocities of a sample of dark matter particles versus their radii (from a sample of 1000 particles in the central kiloparsec of the initial cosmological simulation). Four times are shown: the initial cosmological simulation, 100kyr before the introduction of the black hole particle (12 levels of refinement), 100kyr, and 900kyr after the introduction of the black hole particle.

\subsubsection{Stability Against Artificial Fragmentation}

Finally, it is well known that poor resolution in hydrodynamics codes can lead to artificial fragmentation in the gas. Truelove et al. (1997) investigated these numerical instabilities and found that a simulation must resolve the Jeans length to avoid artificial fragmentation. In addition to the mass criteria described above, the ART code's refinement scheme meets the Jeans condition of Truelove et al. (1997) on the maximum level of refinement (which increases gradually during the initial zoom-in episode), requiring that $\Delta x / \lambda_{\mathrm{J}}<0.25$, where $\Delta x$ is the resolution, or cell size, and $\lambda_{\mathrm{J}}$ is the local thermal Jeans length. In addition, as the mesh refines, rapidly collapsing structures can cause numerical instabilities at 
steep density gradients. The ART code implements artificial pressure support (as described in Machacek et al. (2001)) on the maximum level of refinement, in order to avoid over-collapsing of structures. 


\section{Chapter 5}

\section{RESOLVING GAS DYNAMICS IN THE CIRCUMNUCLEAR REGION OF A SIMULATED DISK GALAXY}

\subsection{Introduction}

The study we present here follows the evolution of the circumnuclear region in a typical galaxy environment at $z=4$ of the self-consistent cosmological simulation described in Chapter 4. We have specifically chosen a simulated galaxy that will evolve into a typical $L_{*}$ galaxy at $z=0$. This particular $z=4$ cosmological simulation was chosen for this study because it contains a galaxy that has not been disturbed by a major merger since it merged with a galaxy with $25 \%$ of its mass at $z \approx 6$. The time since this dynamically active period $(\approx 600$ million yr) is significantly longer than the dynamical time of the galactic disk at $z \approx 4$ $(\approx 15$ million $\mathrm{yr})$. The galaxy is, however, far from quiescent, having grown in mass by $\approx 25 \%$ from $z=5$ to $z=4$ via minor mergers and accretion from its environment. The simulation follows the development of instabilities in the circumnuclear disk, which may drive the transport of matter and angular momentum from large to small scales. The present chapter focuses on the structure of the galaxy and the development of instabilities which may be responsible for driving matter and angular momentum transport within the galaxy. Chapter 6 explores the time evolution of such transport, specifically the evolution of the accretion rates of mass and angular momentum. 
The organization of the present chapter is as follows. In $\S 5.2$ we describe the features of the highly resolved galaxy in a single zoom-in episode at $z=4$, including a stability analysis of the disk. $§ 5.3$ describes an alternative method for measuring the viscosity in the simulations and $\S 5.4$ addresses angular momentum conservation. $§ 5.5$ examines the potential role of physics missing from the present simulations. Finally, $§ 5.6$ contains a summary of our conclusions and their interpretation.

\subsection{Matter Transport in the Circumnuclear Region}

\subsubsection{Structure of the Circumnuclear Disk}

Volume-rendered images of the gas density in the $z=4$ simulation, such as those in Figure 4.4, clearly show the spiral disk structure of the galaxy. The gas disk extends inward all the way to subparsec scales, which are the smallest scales resolved in our simulation. ${ }^{1}$ The geometric structure of the circumnuclear region is best illustrated by the eigenvalues of the inertia tensor, calculated in spherical shells (of widths 0.1dex) around the black hole. The inertia tensor is given by

$$
I_{j k}=\sum_{\alpha=1}^{N} m_{\alpha} x_{j}^{\alpha} x_{k}^{\alpha},
$$

where $m_{\alpha}$ is the gas mass of cell $\alpha$ at radius $r_{\alpha}=\left|\boldsymbol{x}_{\alpha}\right|$ inside a spherical shell containing $N$ cells and centered on the black hole. The eigenvalues of the inertia tensor define the principal axes of each shell, and their ratios describe the shape. Figure 5.1 shows the average radial profile of the ratios of the inertia tensor's eigenvalues. Above $0.1 \mathrm{pc}, c$ is significantly smaller than $b$ over several orders of magnitude in radius, indicating a disk structure. However, the fact that $b / a$ is

\footnotetext{
${ }^{1}$ In particular, the simulated disk does not contain the commonly observed toroidal structure in the inner few parsecs of the galaxy. The failure of our simulation to reproduce the AGN torus may be the result of additional physics still missing in the simulation, as we discuss in $\$ 5.5 .1$.
} 




Figure 5.1 Ratios of eigenvalues of the inertia tensor in spherical shells centered on the black hole. The ratio $c / a \ll b / a$, indicating a disk structure.

significantly less than 1 in parts of the disk indicates that the disk is not axially symmetric, and that it has large-scale structures, such as spiral waves and bars.

The gas disk in the simulation is fully rotationally supported, with the tangential component of velocity, $v_{\mathrm{t}}$, dominating over the radial component, $v_{r}$, by at least an order of magnitude, as illustrated in Figure 5.2. The top panel of Figure 5.2 shows the ratio of the radial to the tangential component of velocity, which is small throughout much of the disk, as the motion of the gas is almost entirely rotational. The average radial velocity is negative, indicating the inflow of gas. The bottom panel shows the tangential component of velocity in units of a quasi-Keplerian velocity, $[G M(r) / r]^{1 / 2}$, determined by the interior total mass, $M(r)$, at each radius. The velocity is close to being Keplerian, but since the mass distribution in the disk resembles a flattened ellipsoid, rather than a spherical 
distribution, the rotation is slightly super-Keplerian.

A remarkable feature of the circumnuclear disk is that the average gas density profile, measured within spherical shells centered on the black hole, follows an almost perfect power law with little evolution in time. Figure 5.3 shows the gas density profile from two different snapshots of the simulation, as well as the average of the profile over an $\approx 550,000 \mathrm{yr}$ period. Both the snapshots and the average profiles of the gas density increase by $\approx 8$ orders of magnitude in the inner 100 pc of the simulation, obeying a steep power law with slope $-8 / 3$. The stability of the gas density profile indicates that the disk is in a quasi-stationary state on timescales of several hundred thousand years. Figure 5.3 also shows the dark matter and stellar mass density profiles. In the central kiloparsec of the galaxy, gas comprises $\sim 62 \%$ of the mass $\left(\sim 2.3 \times 10^{10} h^{-1} M_{\odot}\right.$ total in the central kiloparsec), dominating the dynamics of the disk. In contrast, the stellar and dark matter populations comprise $\sim 13 \%$ and $\sim 25 \%$ of the galaxy's mass inside the central kiloparsec. The disk is extremely gas-rich at $z=4$, because the galaxy is still actively growing and has not yet formed all of its stars. Interestingly, we find that both the stellar and dark matter profiles measured in the central $\sim 200 \mathrm{pc}$ of the galaxy follow simple $\propto r^{-2}$ power laws. The profiles match those predicted for the adiabatic contraction of dark matter from an NFW profile (Navarro et al., 1997), using the model of Gnedin et al. (2004), all the way down to the 1pc scale.

Figure 5.4 shows that the rms velocity dispersion of the gas (measured between neighboring cells and therefore depending on the resolution) greatly exceeds the sound speed in the inner 100pc, indicating supersonic turbulence in the disk. Supersonic turbulence decays on a dynamical timescale, so the persistence of the turbulence in the circumnuclear disk of the galaxy indicates a driving mechanism, which is addressed in the following sections. The mean sound speed of the gas indicates a cold molecular gas disk within the central 100pc of the simulated galaxy. 


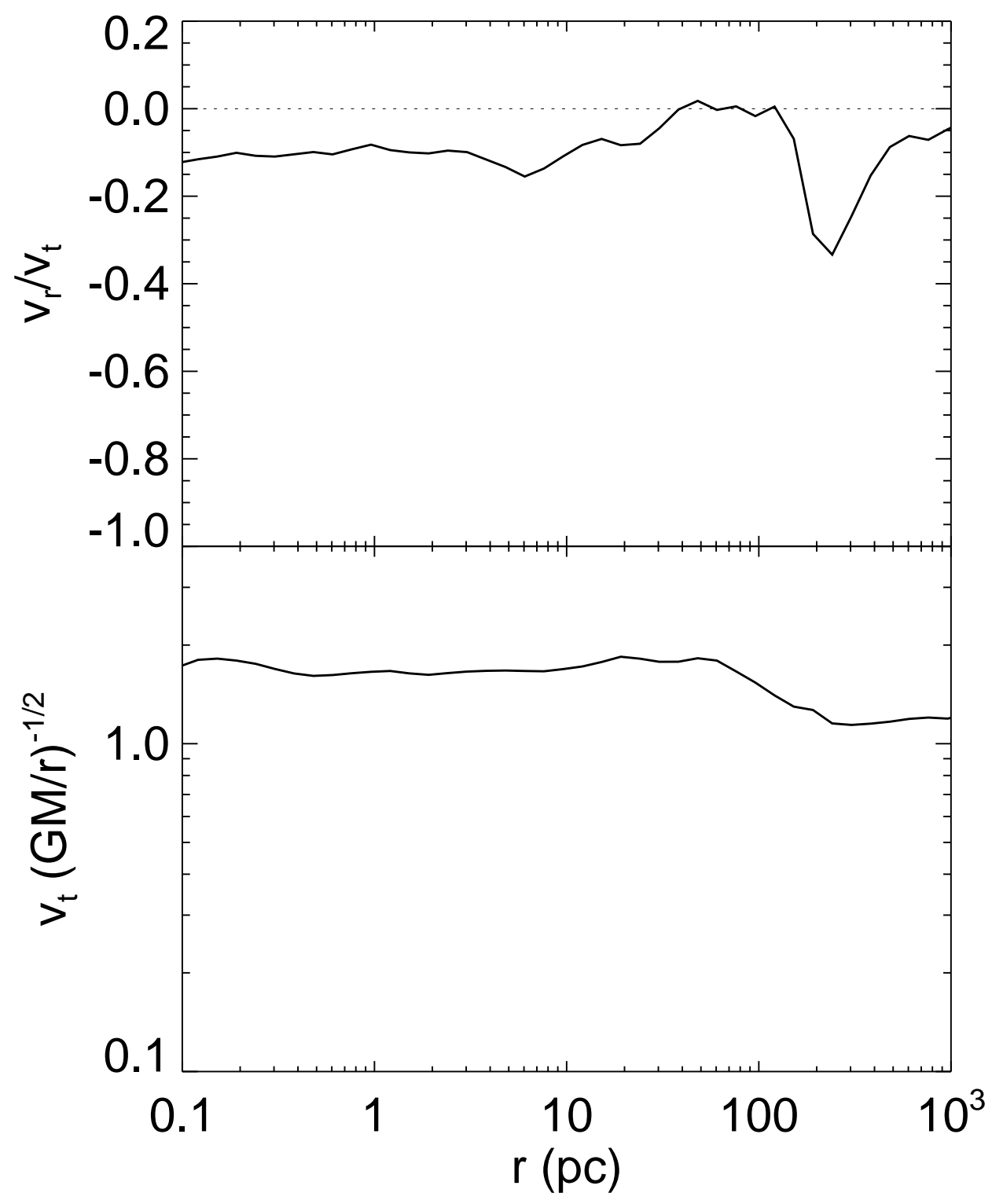

Figure 5.2 Top: Ratio of radial and tangential velocity components of the gas, each averaged over 550,000 yr. Bottom: Average tangential velocity component in units of the average quasi-Keplerian velocity. 


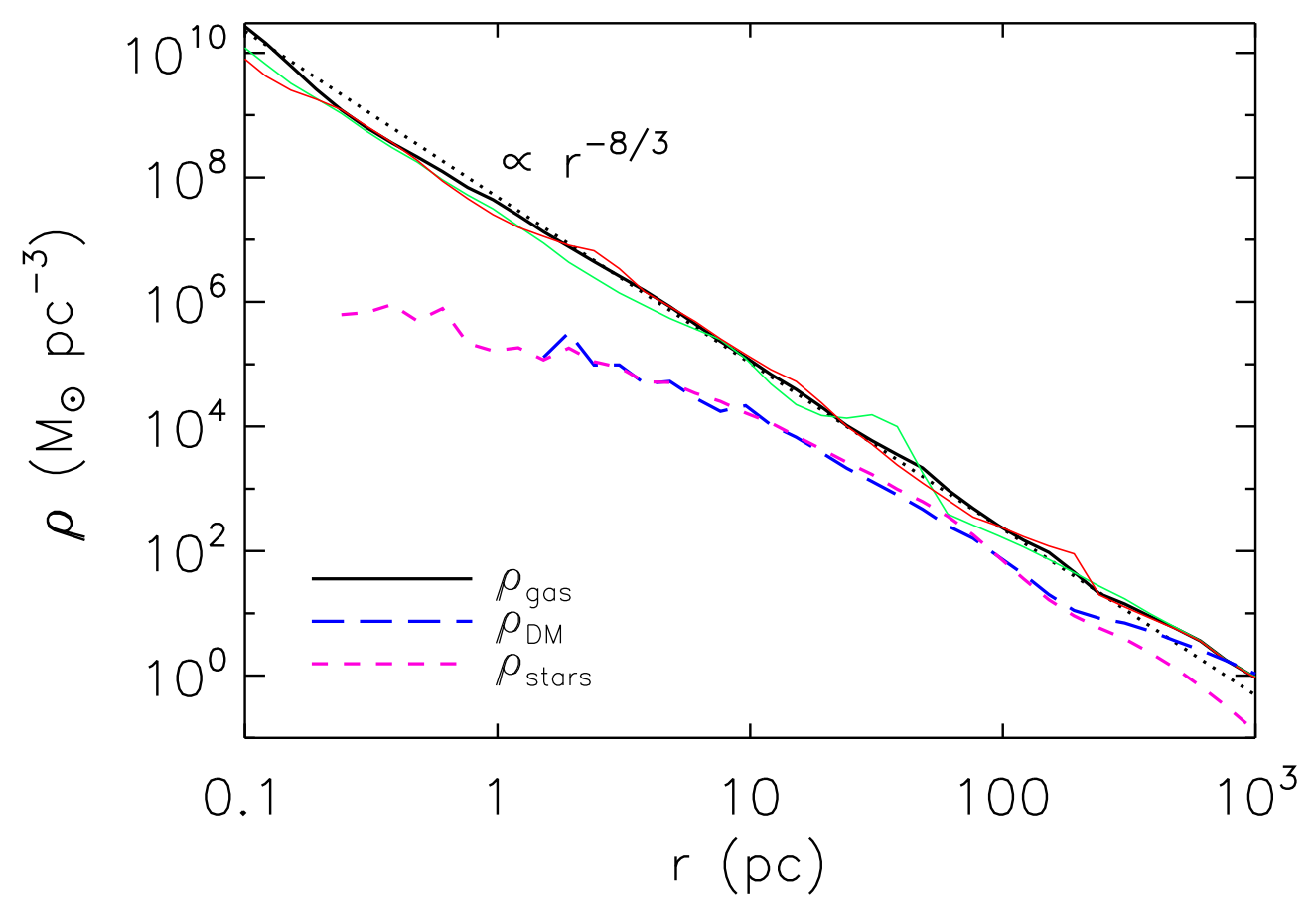

Figure 5.3 Radial profiles of the gas, dark matter, and stellar mass density. Snapshots of the gas density are shown at 100,000 and 500,000 yr after the initial refinement (thin solid lines; green and red respectively), in addition to a 550,000 yr average (thick solid line). The snapshots do not show much variation from the averaged profile, demonstrating that on timescales of several hundred thousand years, the disk is in a quasi-stationary state. The dotted line shows a power law with slope $-8 / 3$ for comparison, which matches the gas density slope well. The dark matter and stellar mass density profiles are shown by the long-dashed blue and short-dashed magenta lines, respectively. In the inner $\sim 200 \mathrm{pc}$, the slope of the dark matter and stellar density profiles is approximately -2 consistent with the adiabatic contraction model.

The floor in the sound speed shown in Figure 5.4 is determined by the minimum temperature to which our adopted cooling rates (computed using the CLOUDY package) apply. The mean sound speed is computed for cells with $T<20,000$ $\mathrm{K}$, because the spherical averages shown in Figure 5.4 include the low-density gas infalling perpendicular to the plane of the galactic disk and shock-heated to high temperatures $\left(>10^{6} \mathrm{~K}\right)$. In addition, turbulence inside the disk produces 


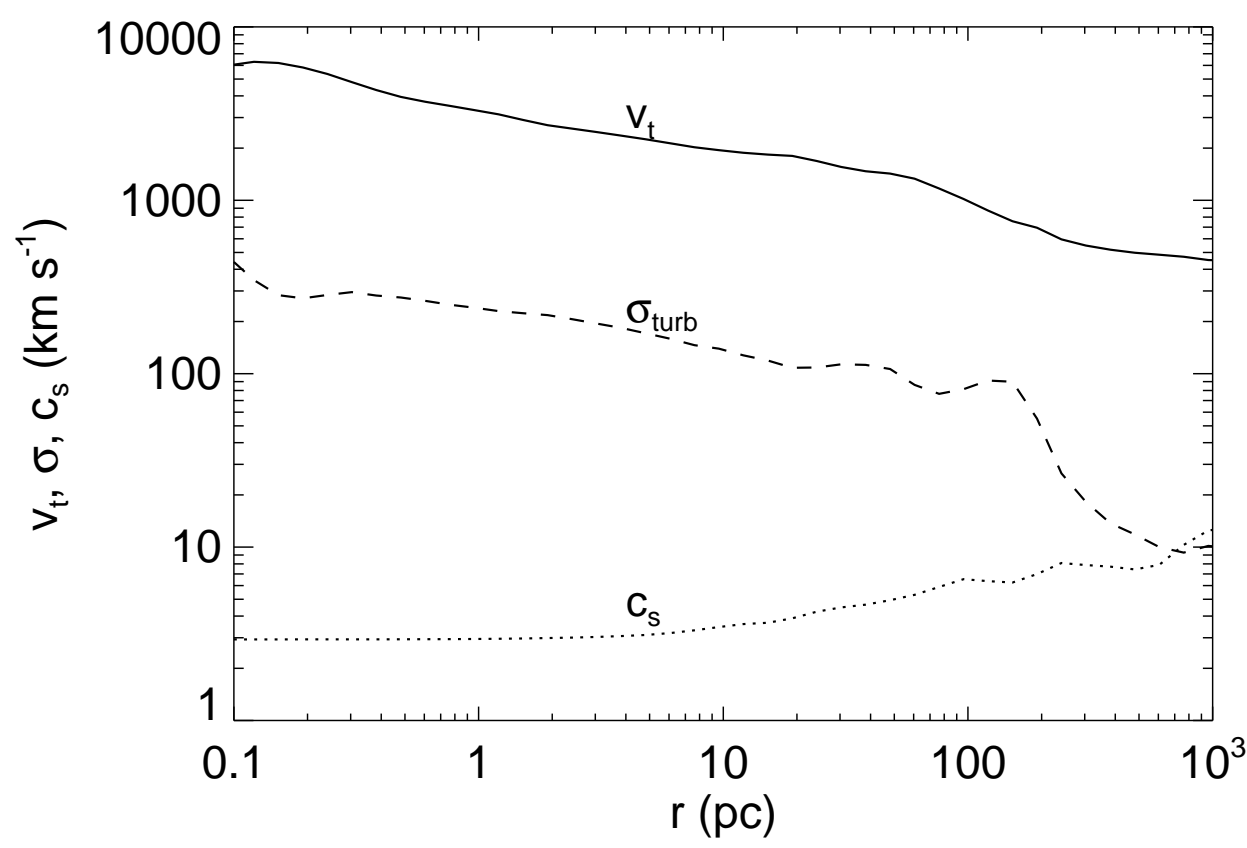

Figure 5.4 Comparison of the radial profiles of the tangential velocity component of the gas (solid line), its rms velocity dispersion or turbulent velocity (dashed line), and the mean sound speed, computed in cells with $T<20,000 \mathrm{~K}$ (dotted line), time-averaged over 550,000 yr. The sound speed is low, indicating cold molecular gas within the central 20pc.

localized shocks that briefly heat individual cells to similarly high temperatures. The inclusion of shock-heated gas produces a broad temperature distribution and raises the mean temperature of the gas so that it does not effectively describe the typical sound speed of the gas in the disk. Therefore, the mean is computed over a temperature range that selects cells in the disk while excluding atypical cells, resulting in a more representative quantity for describing the thermal properties of the disk.

\subsubsection{Angular Momentum Transport}

Averaged over a sufficiently long time, the disk can be considered to be approximately azimuthally symmetric. In addition, the rotational velocity of the 
disk dominates over other velocity components, the local velocity dispersion, and the sound speed, as demonstrated in Figure 5.4. Therefore, the circumnuclear disk in the simulation is well approximated by a thin, rotationally supported, viscous, molecular gas disk. Conservation of momentum in the disk gives the following equation:

$$
\frac{d}{d t}(\rho \boldsymbol{v})=-\nabla P_{\text {tot }}-\rho \nabla \Phi
$$

where $\boldsymbol{v}$ is the velocity, which is dominated by the tangential (or rotational) component, $\Phi$ is the gravitational potential of the disk, and $P_{\text {tot }}$ describes the pressure as a combination of an isotropic thermal pressure and a dynamic pressure, so that

$$
P_{\mathrm{tot}}^{i j}=P \delta_{i j}+\rho v_{i} v_{j}
$$

where $v_{i, j}$ are individual components of the velocity at a given location. The dynamic pressure term represents shear stresses, which generate torques in the disk. Equation 5.2 can be rewritten in terms of the angular momentum density of the disk, given by $\boldsymbol{J}=\rho(\boldsymbol{r} \times \boldsymbol{v})$, and integrated over the interior volume of the disk so that

$$
\begin{aligned}
\frac{d}{d t}\left(\int \boldsymbol{J} d V\right)=\frac{d \boldsymbol{L}}{d t} & =-\int\left(\boldsymbol{r} \times \nabla P_{\mathrm{tot}}\right) d V-\int \rho(\boldsymbol{r} \times \nabla \Phi) d V \\
& =-\left(\boldsymbol{G}_{P}+\boldsymbol{G}_{g}\right)
\end{aligned}
$$

describes the transport of angular momentum through the disk by torques arising from gravitational forces and shear stresses. Since the disk is rotationally supported, the angular momentum is dominated by a single component throughout much of the circumnuclear region. Therefore, the transport of angular momentum 
is governed by the components of $\boldsymbol{G}_{g}$ and $\boldsymbol{G}_{P}$ aligned with the angular momentum vector $\boldsymbol{L}$, which we will simply call $G_{g}$ and $G_{P}$. The above torques can be measured directly from the simulation and compared to the viscous torque described in Pringle (1981) for a thin, azimuthally symmetric, viscous disk, which is parameterized as

$$
G_{\nu}(R, t)=2 \pi \nu \Sigma R^{3} \frac{\partial \Omega}{\partial R}
$$

where $\nu$ is the coefficient of turbulent viscosity, $\Sigma$ is the surface density (given by $2 \rho r)$, and $\Omega=v_{\mathrm{t}} / r$ is the angular velocity. The torques described by the dynamic pressure term of Equation 5.3 and by Equation 5.5 are generated by the turbulent motions of the gas and necessarily vanish in the case of solid-body rotation. We estimate the coefficient of turbulent viscosity, $\nu$, using a characteristic velocity and length scale, which depend on the properties of the turbulence, as is described in more detail in Sections 5.2.3 and 5.3. The viscosity in the disk is presumed to arise primarily from turbulence, rather than numerical effects. The assumption is valid for the duration of the simulation $(\sim 550,000 \mathrm{yr})$, over which time the density profile and angular momentum profile remain rather steady (see Fig. 5.3 above, and Sec. 5.4, below), indicating that there is no systematic numerical dissipation at work.

Figure 5.5 shows a comparison of the average gravitational and pressure torques measured from the simulation, and the viscous torque given by Equation 5.5. The gravitational and pressure torques are summed over the disk interior to radius $r$, projected along the direction of the disk's angular momentum vector (also computed over the interior), and then averaged over the duration of the simulation $(550,000 \mathrm{yr})$. The viscous torque of Equation 5.5 is calculated from quantities radially averaged over spherical shells and (also time-averaged). The 




Figure 5.5 Comparison of different torques responsible for angular momentum transport. The red symbols indicate negative torques. The average rate of angular momentum transport per orbital period is also shown for comparison (solid black curve).

average gravitational torque is positive, corresponding to outward transport of angular momentum through the disk. The gravitational torque dominates over the torque generated by shear stresses throughout much of the circumnuclear region, although both torques are significant. The viscous torque, $G_{\nu}$, constructed from Equation 5.5 is comparable in magnitude to the pressure torque, $G_{P}$, however the expression for $G_{\nu}$ does not capture the chaotic behavior of the stress term, and therefore agreement between the two torques is questionable and warrants further investigation. Although gravitational torques appear to be the main mechanism of angular momentum transport, viscous torques may contribute to the stability of the disk, which will be explored in subsequent sections. 


\subsubsection{Disk Stability and the Source of Turbulence}

In $§ 5.2 .1$ we show that the simulated galaxy contains a self-gravitating, turbulent, cold molecular gas disk within the central $\approx 100$ pc. Such disks are susceptible to instabilities and fragmentation. In the snapshots shown in Figure 5.6, the presence of instabilities is illustrated by waves moving through the simulated disk. The snapshots trace the evolution of disk structure on scales of $\approx 125$, and $\approx 12.5 \mathrm{pc}$ (inset). The rotational period of the disk at the outer radius is such that the disk has undergone at least one full rotation (several more at smaller radii) over the timescales followed by the present simulation. The panels show the somewhat chaotic formation and re-formation of spiral structures on these scales, in contrast with the more ordered spiral structure seen on kiloparsec scales (Figure 4.4). This section includes an analysis of the behavior of structures in the disk on the scales shown in Figure 5.6.

The Toomre $Q$-parameter describes the stability of the disk against linear perturbations and is given by $Q=\sigma_{\text {turb }} \kappa / \pi G \Sigma$, where $\kappa$ is the epicyclic frequency and $\Sigma$ is the surface density of the gas, both determined locally (Toomre, 1964; Goldreich \& Lynden-Bell, 1965). Although the disk is cold and the sound speed is low, the rms velocity dispersion is substantially higher than the sound speed inside $100 \mathrm{pc}$, indicating that the gas is turbulent. Therefore, in place of the sound speed typically used in the definition of the Toomre $Q$-parameter, we have substituted a turbulent velocity, given by the quadrature sum of the sound speed and the rms velocity dispersion of the gas. The disk is, for the most part, quasi-Keplerian (as described in $\S 5.2 .1$ ), so that the epicyclic frequency $\kappa$ is proportional to the angular speed of the disk, $\Omega$.

The top panel of Figure 5.7 shows the Toomre $Q$-parameter for the simulated disk, corresponding to the region shown in Figure 5.6. Where $Q<1$ the 


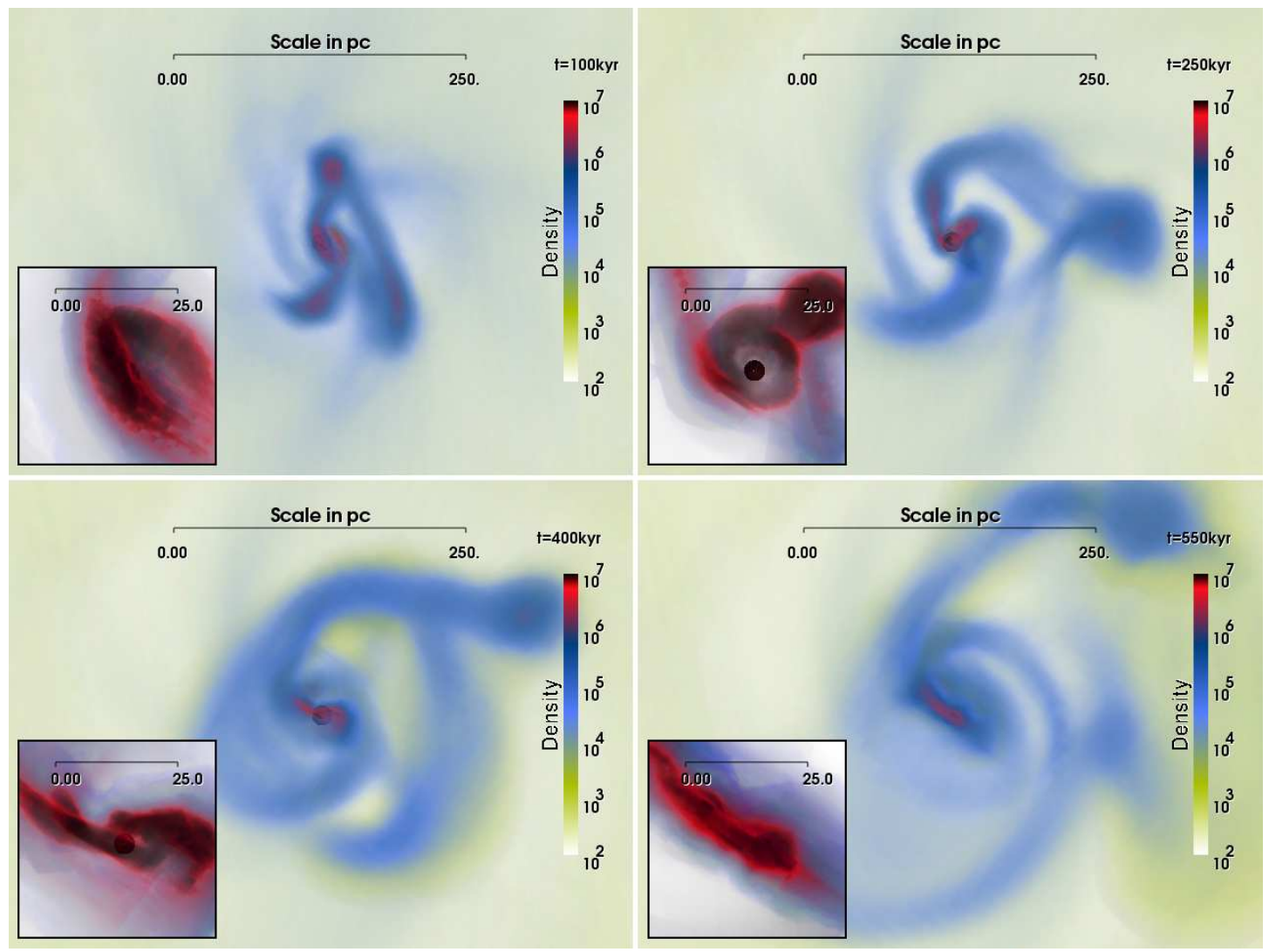

Figure 5.6 Evolution of global instabilities in the disk over time, on scales of $\approx 125 \mathrm{pc}$ and $\approx 12.5 \mathrm{pc}$ (inset). Each panel shows a volume rendering of the gas density $\left(\right.$ in $\mathrm{cm}^{-3}$ ) at a different epoch. The similarities between the appearances of the disk on the two different scales indicate the hierarchical structure of the disk. 
disk is susceptible to local gravitational instabilities resulting from axisymmetric perturbations. For $Q>1$ the disk is likely to be stable against axisymmetric perturbations but is still susceptible to instabilities arising from nonaxisymmetric perturbations, which are less stable than radial perturbations (Polyachenko et al., 1997). For the density profile shown in Figure 5.3, the disk becomes stable for $Q>2$. The shaded region in Figure $5.7(1<Q<2)$ therefore represents marginally stable values of the $Q$-parameter, where the disk still might become unstable. Figure 5.7 suggests that the disk in the simulated galaxy lies mostly in the region of marginal stability inside $\approx 1 \mathrm{kpc}$. In the case of axisymmetric perturbations, the fastest growing unstable mode corresponds to the scale $\lambda_{\text {fast }, r}$, at which $d \omega^{2} / d k=0$ (where $\omega$ is the angular frequency of waves in the disk), given by

$$
\lambda_{\text {fast }, r}=\frac{2 \sigma_{\text {turb }}^{2}}{G \Sigma}
$$

Using the condition for marginal stability from Polyachenko et al. (1997), the fastest growing mode for all modes (axisymmetric and nonaxisymmetric), $\lambda_{\text {fast,all }}$, is given by

$$
\lambda_{\text {fast }, \text { all }}=\frac{\lambda_{\text {fast }, r}}{2}=\frac{\sigma_{\text {turb }}^{2}}{G \Sigma} .
$$

The bottom panel of Figure 5.7 shows the ratio $\lambda / r$ for each of the fastest growing modes. In the inner $100 \mathrm{pc}$, the scales of the fastest growing modes are smaller than the radius by only a factor of a few, implying that the disk is stable on scales $\lambda \ll r$ (at least in the linear regime). This is an indication that perturbations in the disk operate on a range of scales, but always on scales that are an appreciable fraction of the size of the system, driving global instabilities, which generate turbulence on smaller scales. The disk remains locally stable all the way 
into a region less than $1 \mathrm{pc}$ from the black hole. There is no catastrophic fragmentation into clumps small enough to form stars, so that angular momentum transport may continue uninterrupted by bursts of star formation. The result is somewhat similar to that of Wise et al. (2007), who find that the central region of a simulated protogalaxy, which is turbulent and only partially rotationally supported, becomes gravitationally unstable and collapses without first fragmenting into clumps.

By dimensional analysis, the turbulent kinematic viscosity $\nu$ discussed in $\S 5.2 .2$ can be described by $\lambda \sigma_{\text {turb }}$, where $\lambda$ is a characteristic scale for turbulence and $\sigma_{\text {turb }}$, the turbulent velocity shown in Figure 5.4, is a characteristic velocity. It is natural to identify the characteristic length scale with the length scale corresponding to the fastest growing unstable mode, $\lambda_{\text {fast }, r}$, so that $\nu \sim \lambda_{\text {fast }, r} \sigma_{\text {turb }}$.

The conditions in the circumnuclear disk are potentially conducive to gaseous bar formation, the conditions for which have been studied extensively for different geometries and physical conditions, resulting in a variety of criteria (e.g. Ostriker \& Peebles, 1973; Efstathiou et al., 1982; Christodoulou et al., 1995a,b; Bottema, 2003; Wyse, 2004). A comparison with the criterion of Ostriker \& Peebles (1973), which characterizes stability in terms of the ratio $t=T /|W|$ of kinetic to gravitational potential energy, indicates that the disk is both secularly and dynamically unstable to bar formation on all scales $r \gtrsim 0.1 \mathrm{pc}$ adequately resolved by the simulation. Because of the chaotic and transient behavior of the instabilities, the structures shown in Figure 5.6 do not resemble a single, well-defined bar, but rather a highly perturbed "bar."

Since the Toomre criterion applies only to small linear perturbations and the Ostriker-Peebles criterion describes global modes, the question remains whether nonlinear effects lead to fragmentation on smaller scales, below the resolution of the simulation. The top panel of Figure 5.8 shows the ratio of the local Jeans 


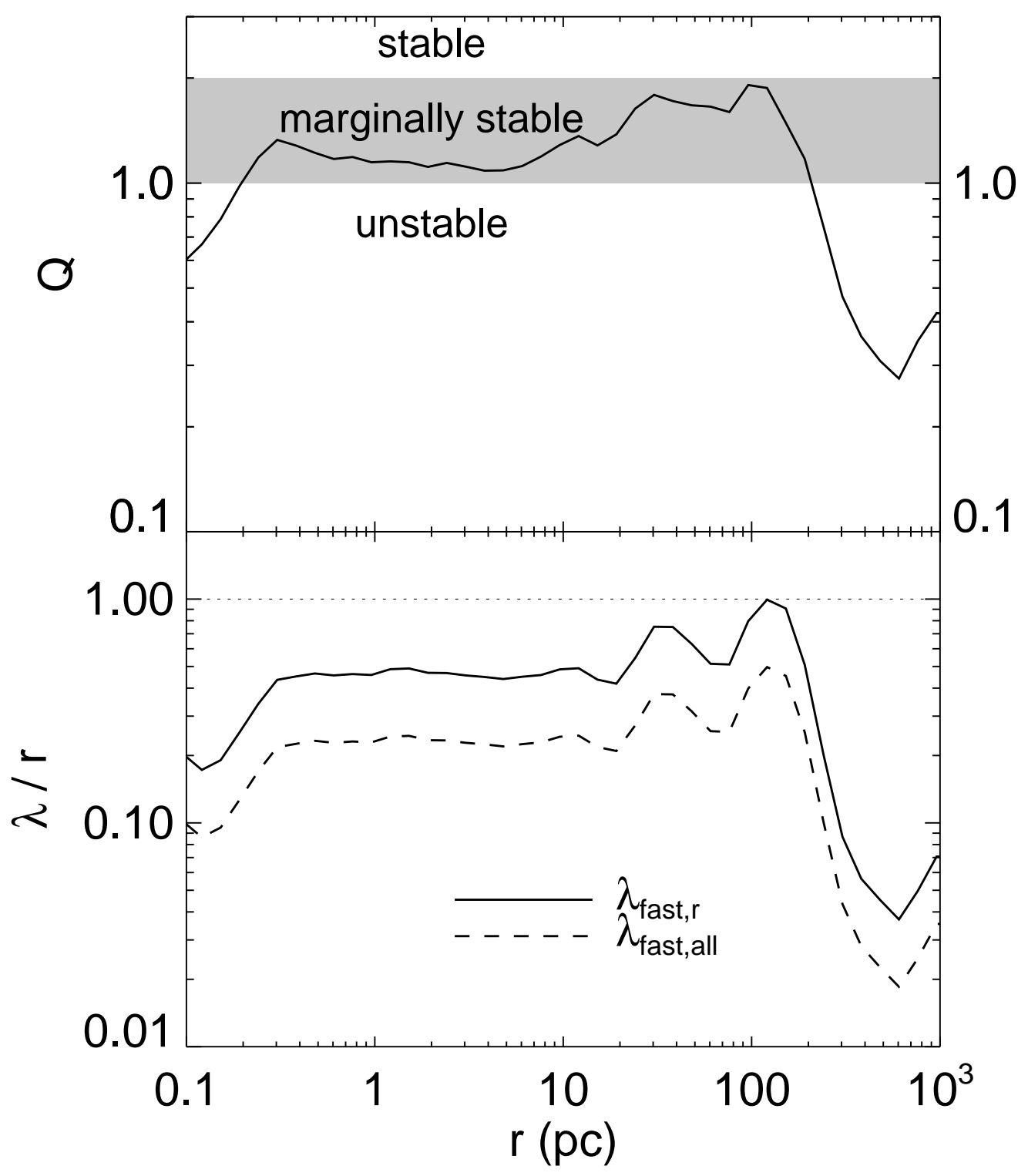

Figure 5.7 Top: Toomre $Q$-parameter. For $Q<1$ the disk is unstable, and for $Q>2$ the disk is stable. In the regime $1<Q<2$, the disk is marginally stable (stable against axisymmetric modes but not nonaxisymmetric modes). Bottom: Average values of the fastest growing unstable mode for axisymmetric perturbations, $\lambda_{\text {fast }, r}$ (solid line), and the fastest growing unstable mode for all perturbations, $\lambda_{\text {fast,all }}$ (dashed line), each divided by radius. The ratio $\lambda / r \gtrsim 0.1$ throughout the circumnuclear disk, so there is no catastrophic fragmentation down to small scales. 
length of the gas, $\lambda_{\mathrm{J}}$, to cell size, $\Delta x$, for all simulation cells in the circumnuclear disk (with temperatures less than $10^{3} \mathrm{~K}$ ). In most cells, the Jeans length is larger than the cell size, preventing the gas from fragmenting into subcell-sized clouds, ultimately leading to star formation. The Truelove criterion for preventing numerical fragmentation is enforced on the maximum level of refinement (level 20) only, so there are no numerical restrictions to prevent gas from collapsing all the way to level 20. However, only the central subparsec part of the disk reaches levels 19 and 20, as shown by the histogram in Figure 5.8 (bottom), indicating that the disk is stable to nonlinear effects. While some cells in the disk have $\lambda_{\mathrm{J}}<\Delta x$ and may form stars, Figure 5.8 demonstrates that most of the disk is stable against collapse, and that there is no widespread burst of star formation. The few cells with $\lambda_{\mathrm{J}} / \Delta x<1$ may correspond to resonances where the pattern speed of waves in the disk is the same as the rotational velocity, leading to higher gas densities and possible star formation. However, a detailed analysis of the behavior of these resonances with time is beyond the scope of this thesis.

\subsubsection{The Nature of the Turbulence}

Turbulence has been widely studied in the modeling of the ISM of galaxies and star-forming regions (for recent reviews, see Mac Low \& Klessen, 2004; McKee \& Ostriker, 2007). It is important to understand the nature of the turbulence in the present simulated galaxy, as it plays a key role in the disk properties. The global instabilities arising in the simulated circumnuclear disk generate turbulence on a range of scales, which acts to stabilize the disk against collapse.

Turbulence dissipates energy in such a way that the turbulent velocity scales as $\sigma_{\text {turb }} \propto \lambda^{q}$, where $q=1 / 3$ for incompressible, sub-sonic (Kolmogorov) turbulence and $q=1 / 2$ for compressible turbulence in the zero-pressure limit (Burgers turbulence). The supersonic turbulence in the simulated disk falls between these 


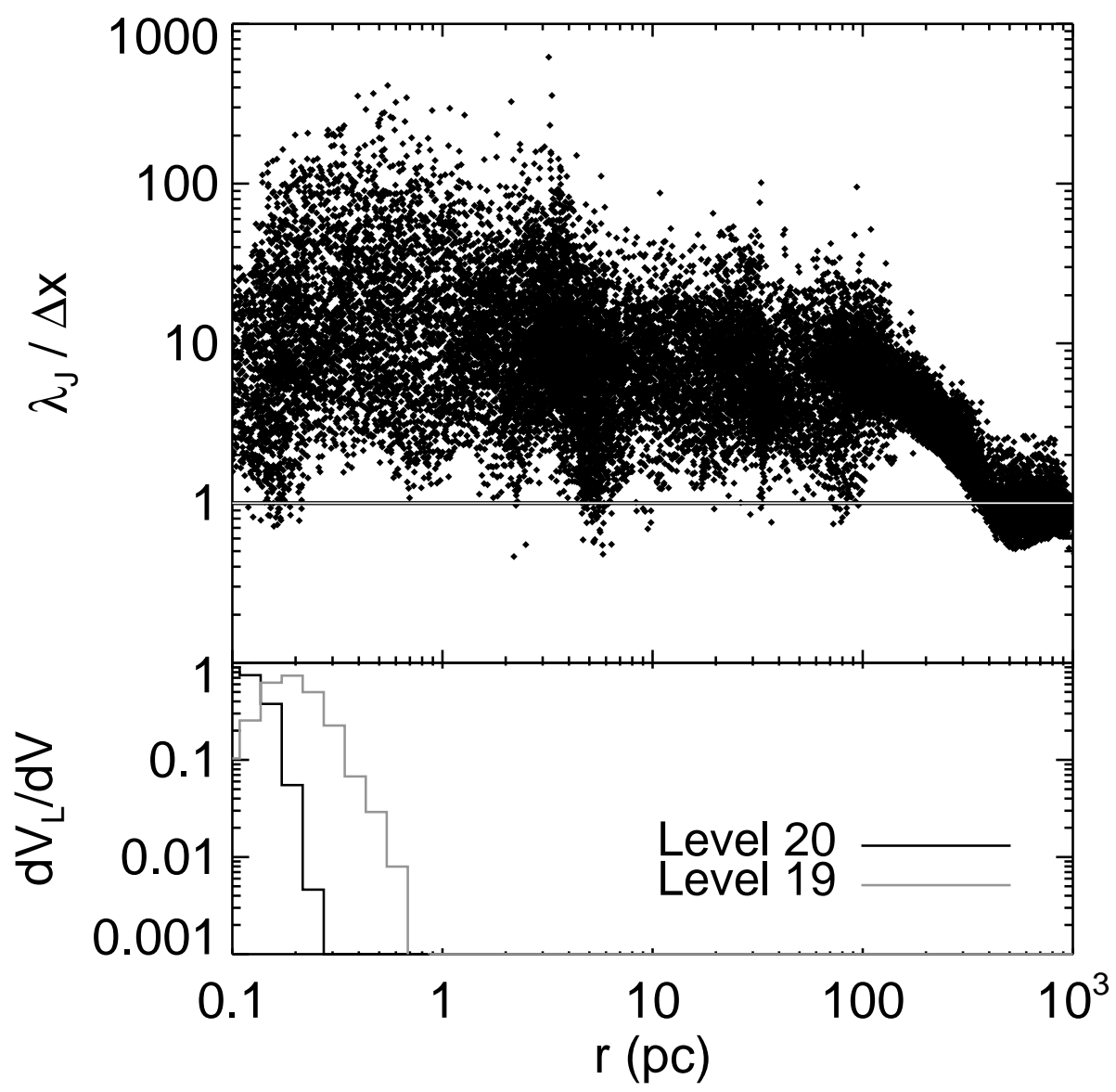

Figure 5.8 Top: Scatter plot showing the ratio of the local Jeans length, $\lambda_{\mathrm{J}}$, to the cell size, $\Delta x$ at 300,000 yr after the initial refinement. Only cool cells with temperatures less than $10^{3} \mathrm{~K}$ are shown. Bottom: Histogram showing the volume of level 20 and level 19 cells as a function of radius. The simulation only refines to the maximum levels in the center of the circumnuclear disk.

two limits. In AMR simulations, it is straightforward to measure the turbulent velocity on different scales because the information on different levels of refinement is readily available. However, the measurements are only accurate to within a factor of 2 in scale because the cell size decreases by a factor of 2 with each refinement. The approximate scaling of the turbulence is shown in the top panel of Figure 5.9. The figure shows the turbulent velocity (given by the rms velocity dispersion between neighboring cells) at two different radii for two different sim- 
ulation runs. The first is the fiducial run described in the previous sections, and the second is a short portion of the fiducial run, with a more aggressive refinement criterion on levels 13 and below, given by $m_{\mathrm{r}}^{\text {level-10 }} \max \left[0.5^{\text {level-12 }}, 0.125\right]$ (where $m_{\mathrm{r}}$ is an empirically determined parameter defined in $\left.\S 4.3\right)$. The more aggressive run probes smaller scales at a given radius, in order to capture the scale of the turbulence. The turbulent velocities shown in Figure 5.9 demonstrate the scaling of the turbulence down to small scales. It is difficult to determine the precise slope of the scaling using measurements from the present simulation because of the limitation imposed by the refinement scheme. While a more precise characterization of the turbulence spectrum is beyond the scope of the present simulation, Figure 5.9 shows that the slope approximately falls between the Kolmogorov and Burgers turbulence limits, as expected.

At scales below the resolution at a given location in the disk, the turbulent velocities in Figure 5.9 level off at constant values because the turbulence scaling can only be measured down to the resolution limit. The more aggressive refinement run (Fig. 5.9, triangles) probes smaller scales for a given radius and therefore levels off at smaller scales. The bottom panel of Figure 5.9 shows the approximate scale at which the flattening of the spectrum occurs for several different radii in the circumnuclear disk of the fiducial run. For comparison, the fastest growing unstable mode, $\lambda_{\text {fast,all }}$, is shown as well. The turbulent velocity measured at the resolution of the simulation is a numerical quantity, but it roughly corresponds

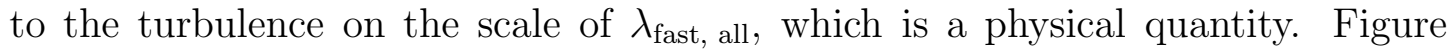
5.9 demonstrates that the simulation resolves all scales that are Toomre $Q$ unstable, supporting the resulting interpretation that instability-driven turbulence maintains the local stability of the circumnuclear disk.

Failing to remove the large scale motions of the disk from the calculation of the turbulent velocity can lead to an overestimate of the turbulent velocity, and 



Figure 5.9 Top: Turbulent velocity as a function of scale (or cell size) at 3 and $8 \mathrm{pc}$ for the fiducial and aggressive refinement runs. The more aggressive refinement run resolves the scaling of the turbulence down to smaller scales. Bottom: Comparison of the smallest resolved scale of turbulence and the fastest growing unstable mode, $\lambda_{\text {fast,all }}$ (where $\lambda_{\text {fast,all }}$ is the same as in Figure 5.7). 


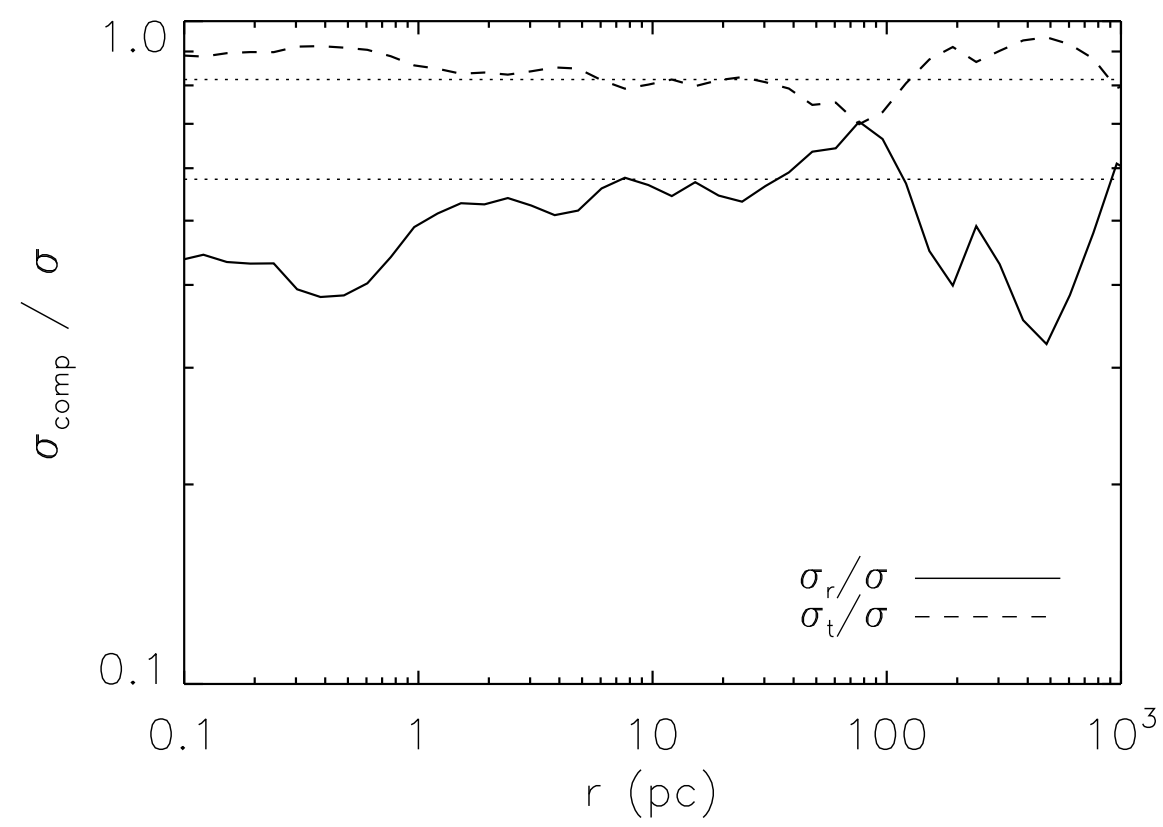

Figure 5.10 Ratio of the individual components of the velocity dispersion to the total dispersion. For an isotropic velocity dispersion, $\sigma_{r}=\sqrt{\sigma^{2} / 3}$ and $\sigma_{t}=$ $\sqrt{2 \sigma^{2} / 3}$. The dotted lines correspond to the isotropic values.

therefore a misinterpretation of the role of turbulence in the circumnuclear disk. The velocity dispersion measured on sufficiently small scales should be isotropic, and independent of the large scale behavior of the disk. Figure 5.10 shows the ratio of individual components of the average velocity dispersion (radial and tangential) to the total dispersion as a function of radius. If the 3-dimensional velocity dispersion is isotropic, then $\sigma_{3 \mathrm{D}}^{2}=3 \sigma_{1 \mathrm{D}}^{2}$, where $\sigma_{1 \mathrm{D}}^{2}=\sigma_{r}^{2}=\sigma_{t}^{2} / 2$. The figure shows that the velocity dispersion we measure is isotropic throughout much of the circumnuclear region $(1 \mathrm{pc} \lesssim r \lesssim 100 \mathrm{pc})$ and therefore provides a reasonable estimate of the turbulent velocity and of the characteristic velocity used to estimate the turbulent viscosity, $\nu$.

The density structure of the disk is also consistent with the description of turbulence in the disk. Supersonic turbulence typically imposes a lognormal den- 


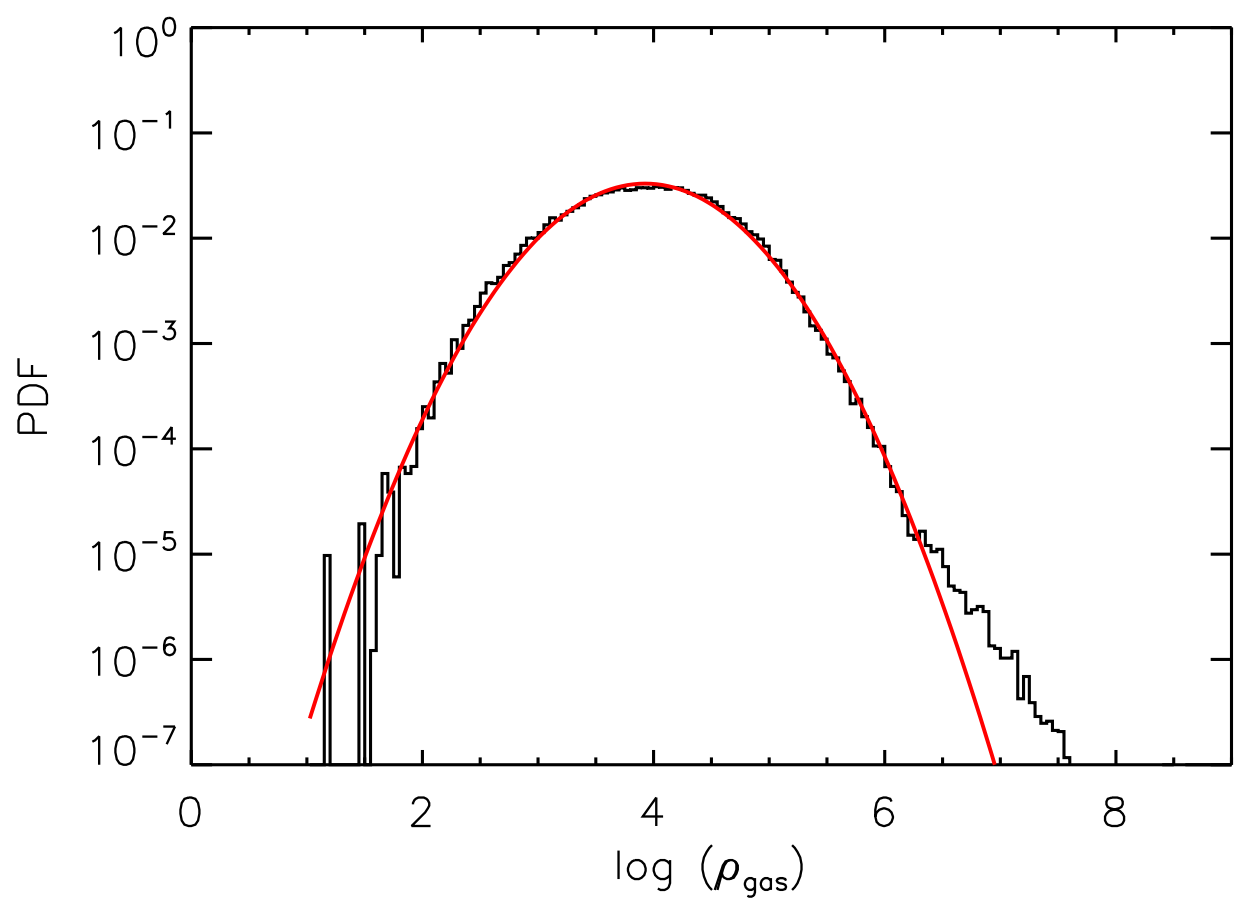

Figure 5.11 Volume-weighted PDF of the gas density in cells at a scale of $\sim 30 \mathrm{pc}$, with temperatures $<10^{3} \mathrm{~K}$ (in order to exclude the hot corona outside the disk), averaged over $\sim 250,000 \mathrm{yr}$. The PDF is well fit by a lognormal distribution over the range $10^{2}<\rho<10^{6} M_{\odot} \mathrm{pc}^{-3}$ with a mean density of $10^{3.9} M_{\odot} \mathrm{pc}^{-3}$ (solid curve).

sity distribution on the gas, as demonstrated by models of turbulence in molecular clouds (e.g. Vazquez-Semadeni, 1994; Passot \& Vázquez-Semadeni, 1998; Wada, 2001; Kritsuk et al., 2007; McKee \& Ostriker, 2007), such as

$$
\frac{d V}{d \rho}=\frac{1}{\rho \sqrt{2 \pi \sigma^{2}}} \exp \left[\frac{-(\ln \rho-\mu)^{2}}{2 \sigma^{2}}\right],
$$

where the mean, $\mu$, is defined as $\overline{\ln \rho}$, and $\sigma$ is the dispersion. Figure 5.11 shows the volume-weighted probability distribution function (PDF), time-averaged over $\sim 250,000 \mathrm{yr}$, for a shell of gas at a radius of 30pc. The PDF is well fit by a lognormal distribution over at least 4 orders of magnitude in density, consistent with models of supersonic turbulence. 


\subsection{Measuring the Viscosity}

Here we address the validity of our estimate of the turbulent kinematic velocity parameter $\nu$, which motivates the interpretation that turbulent transport of angular momentum maintains local stability and the quasi-steady state of the circumnuclear disk. Instead of a direct measurement of the viscosity, we have assumed that the viscosity depends on characteristic velocity and length scales, given by $\sigma_{\text {turb }}$ and $\lambda_{\text {fast, } r}$, respectively. The accuracy of this estimate can be tested by measuring the transport of gas due to turbulent motions.

The ART code does not follow individual parcels of gas across cell boundaries, but rather advected quantities describing the gas. Therefore, in order to follow the turbulent motions of the gas, we introduce a passive scalar into the simulation which has a value of unity inside a spherical region centered on the black hole particle and a value of zero outside this region, effectively "painting" the gas. As the simulation evolves, turbulent motions of the gas cause the profile of the scalar quantity to deviate from its original spherical form. The evolution of the profile depends on the turbulent kinematic viscosity, $\nu$, and in the early stages of diffusion, while the deviations from the initial profile are small, the profile approximately satisfies the linear diffusion equation for the initial conditions,

$$
P(r, t=0)= \begin{cases}1, & \text { if } r<r_{0}, \\ 0, & \text { if } r>r_{0},\end{cases}
$$

(where $r_{0}$ is the radius of the initial sphere), allowing a more direct calculation

of the viscosity. The solution to the linear diffusion equation for the above initial conditions is

$$
P(x, q)=\frac{1}{2}\left[\operatorname{erf}\left(\frac{x+1}{\sqrt{q}}\right)-\operatorname{erf}\left(\frac{x-1}{\sqrt{q}}\right)\right]-\frac{q}{x \sqrt{\pi q}} e^{-\left(1+x^{2}\right) / q} \sinh \left(\frac{2 x}{q}\right)
$$


where

$$
x=\frac{r}{r_{0}} \quad \& \quad q=\frac{4 \nu t}{r_{0}^{2}} .
$$

Figure 5.12 shows samples of the profile evolving from a 30pc sphere for different times $(q)$. The measured profiles agree well with the solution given in Equation 5.10. Starting from several painted regions at 1, 3, 10, 30, and 100pc radii, we follow the evolution of the paint-weighted density profiles and measure the turbulent viscosity, $\nu$, as a function of radius. Figure 5.13 shows the bestfit measurements of $\nu / r$ in relation to the estimate given by $\sigma_{\text {turb }} \lambda_{\text {fast }, r} / r$. The lines in Figure 5.13 show individual snapshots of $\sigma_{\text {turb }} \lambda_{\text {fast }, r} / r$ corresponding to the timescale over which the diffusion was followed. The best-fit measurements match the estimates for $\nu / r$ well, thus lending justification to the estimate for $\nu$ used in the analytic arguments of $\S 5.2 .3$.

\subsection{Angular Momentum Conservation}

In Chapter 4, we presented a separate set of simulations following the collapse of an isothermal, uniformly rotating sphere, in order to test conservation of angular momentum in the ART code. In Figure 5.14 we demonstrate the conservation of angular momentum in the present simulation. As the maximum resolution of the simulation increases, the angular momentum profile initially evolves as torques redistributes angular momentum within the disk. But after reaching the maximum level 20 resolution (the point at which we introduce the black hole particle into the simulation and let it evolve), the profile remains rather steady with time, because the disk has reached a quasi-stationary state. Figure 5.14 shows the angular momentum as a function of enclosed gas mass at several different times during a single zoom-in episode of the simulation. The figure shows the 


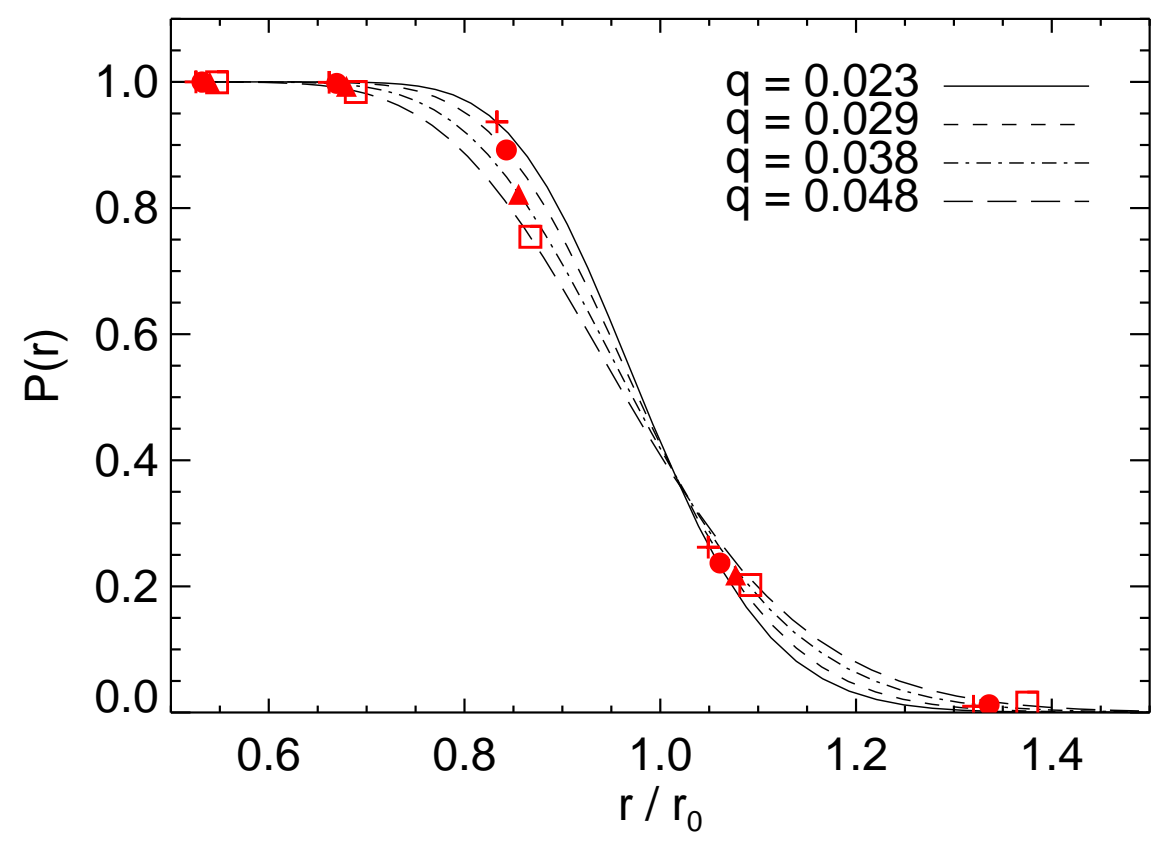

Figure 5.12 Mass-weighted profiles of the passive scalar as a function of radius and evolving over time from a 30pc sphere. The symbols are measurements from the simulation, and the lines are the best fits to Equation 5.10.

evolution of the angular momentum distribution from 200, 000yr before the introduction of the black hole particle, when the simulation has refined to level 11, to $500,000 \mathrm{yr}$ after the introduction of the black hole particle, when the simulation is fully refined and has evolved for several hundred thousand years in a quasi-steady state.

On the scale of the circumnuclear disk, the simulation has made several thousand time steps between $t=100$ and $500 \mathrm{kyr}$, meaning that the simulation has undergone significant evolution on this spatial scale. Numerical effects, accumulating with each time step, would cause deviations in the angular momentum profile. Over longer timescales, the slow inward transport of matter will alter the angular momentum profile. However, on the hundred thousand year timescale we expect the profile to remain rather steady, reflecting the quasi-stationary state of 


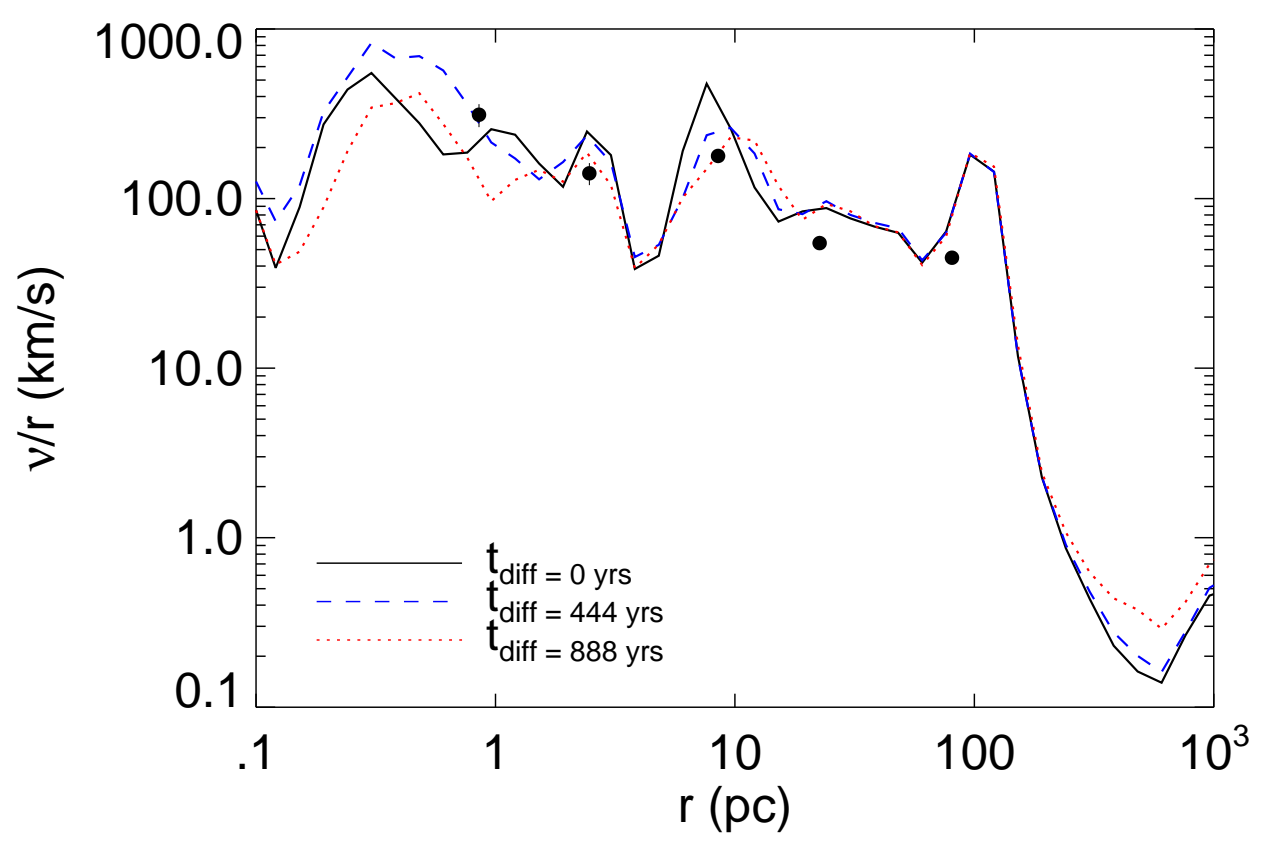

Figure 5.13 Ratio of kinematic viscosity $\nu$ to radius $r$. The lines show estimates for $\nu / r$ given approximately by $\lambda_{\text {fast }, r} \sigma_{\text {turb }}$, for three different snapshots. The points are estimates from a second method measuring the advection of gas at five different radii in the simulation. The snapshots correspond to the profile fits at different times, so the $1 \mathrm{pc}$ point should be compared to the solid line, the next point to the dashed line, and the last three points to the dotted line.

the disk, unless there is anomalous numerical transport of angular momentum. Figure 5.14 shows no systematic deviations in the angular momentum profile after the initial refinement and the corresponding re-distribution of the angular momentum, demonstrating that there is no sign of unphysical numerical angular momentum transport on the timescales of the simulation. On timescales much longer than those simulated here, however, these effects may become important.

\subsection{Possible Effects of Missing Physics}

The simulations presented here do not include all of the potentially important physics for galaxy evolution. Therefore, in this section we discuss the 




Figure 5.14 Angular momentum as a function of enclosed gas mass for several different times. The times are measured from the introduction of the black hole. The vertical dashed lines correspond to enclosed masses on scales of $\approx 10$ and $\approx 1000$ pc. At these scales the simulation has made approximately 230,000 and 3600 steps, respectively (corresponding to the resolution at each scale), between $t=100$ and $500 \mathrm{kyr}$.

potential effect that the physics missing from the simulations might have on our results.

\subsubsection{Optically Thick Cooling}

Cooling in the high-density central region of the galaxy is not treated entirely correctly by the ART code. The column density of the molecular gas in the center is so high that the gas is expected to become optically thick to its own cooling radiation (e.g. Ripamonti \& Abel, 2004). In addition, the presence of dust grains in this region traps radiation and further halts cooling (see Draine \& Lee, 1984; Ossenkopf \& Henning, 1994). Figure 5.15 shows the column density vertically 


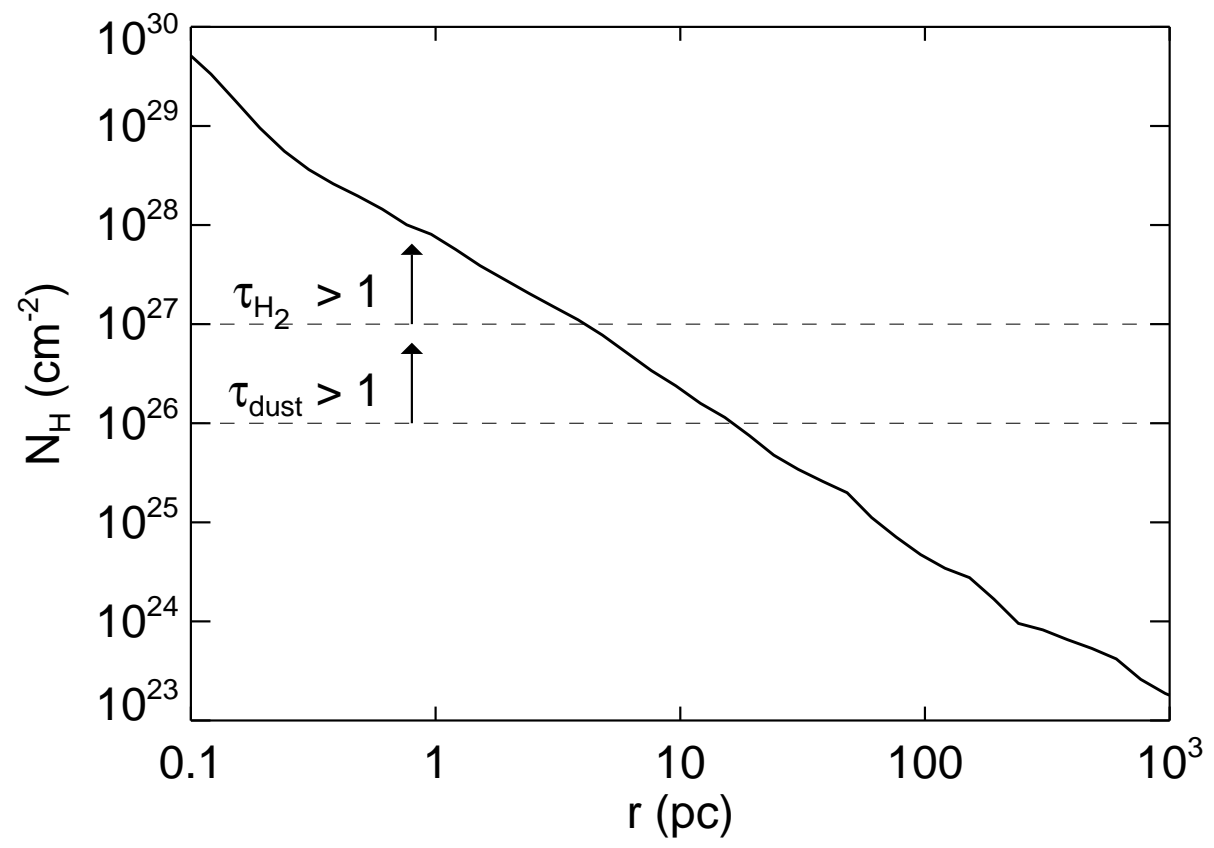

Figure 5.15 Column density of the gas. The horizontal lines show the column densities at which dust and $\mathrm{H}_{2}$ become optically thick to their own cooling radiation.

through the circumnuclear gas disk. Horizontal lines are shown for comparison, corresponding to the column densities at which dust and $\mathrm{H}_{2}$ each become optically thick to their own cooling radiation $\left(\sim 10^{26}\right.$ and $\sim 10^{27} \mathrm{~cm}^{-2}$, respectively, at 0.1 solar metallicity, which is the metallicity of the circumnuclear disk in the simulation at $z=4$ ). In the inner $\approx 10 \mathrm{pc}$, the column density of the gas is large enough that the opacity of dust and molecular gas must be accounted for to accurately describe the cooling in the simulation.

The simulation runs presented here do not include optically thick radiative transfer. This may explain why the gas remains in a thin disk all the way in to subparsec scales and does not resemble the obscuring tori observed in the inner few parsecs of AGNs. Should the opacity of the gas to its own cooling radiation be included, the gas around the mid-plane of the disk would not be able to cool 
and would heat to temperatures corresponding to the energy dumped into the gas by the turbulence. These higher temperatures may be able to provide substantial vertical pressure support in the central few parsecs of the disk, resulting in a thicker, more toroidal structure. ${ }^{2}$ However, it has been suggested that hydromagnetic disk winds, and not hydrostatic pressure support, may be responsible for sustaining the optically and geometrically thick obscuration region, or "obscuring torus," in the nuclei of galaxies (e.g. Konigl \& Kartje, 1994; Elitzur \& Shlosman, 2006). In this case, the inclusion of optically thick radiative transfer may not be sufficient to create a "torus" in our simulations.

In future simulations we plan to remedy this limitation of the present simulation by accounting for the opacity of the high-density region in the center, which will allow us to consistently incorporate radiative feedback from a central source and test the above hypothesis.

\subsubsection{Magnetic Fields}

The ART code does not include magnetic fields, whereas MHD is certainly important for accretion disk physics (see Balbus \& Hawley, 1998, and references therein). It is for this reason that the present study has been restricted to scales larger than $\approx 0.1 \mathrm{pc}$, corresponding to about $10^{4}$ Schwarzschild radii for a black hole of mass $3 \times 10^{7} M_{\odot}$.

At scales larger than the accretion disk, the absence of magnetic fields in the ART code is probably not important. An estimate of the strength that an equipartition magnetic field would need in order to affect the disk on small scales is given by $B_{\mathrm{eq}}^{2} \approx 4 \pi \rho \sigma_{\text {turb }}^{2}$. In order to affect the large-scale dynamics of the gas, the magnetic field would have to have an even larger strength of order $B_{\mathrm{dyn}}^{2} \approx 4 \pi \rho v_{\mathrm{t}}^{2}$.

\footnotetext{
${ }^{2}$ Notice, that the outer, optically thin layers of such a disk would continue cooling efficiently, covering the hot interior with a cold molecular "skin". Such a skin may become Rayleigh-Taylor unstable, leading to fragmentation of the torus into individual clouds.
} 


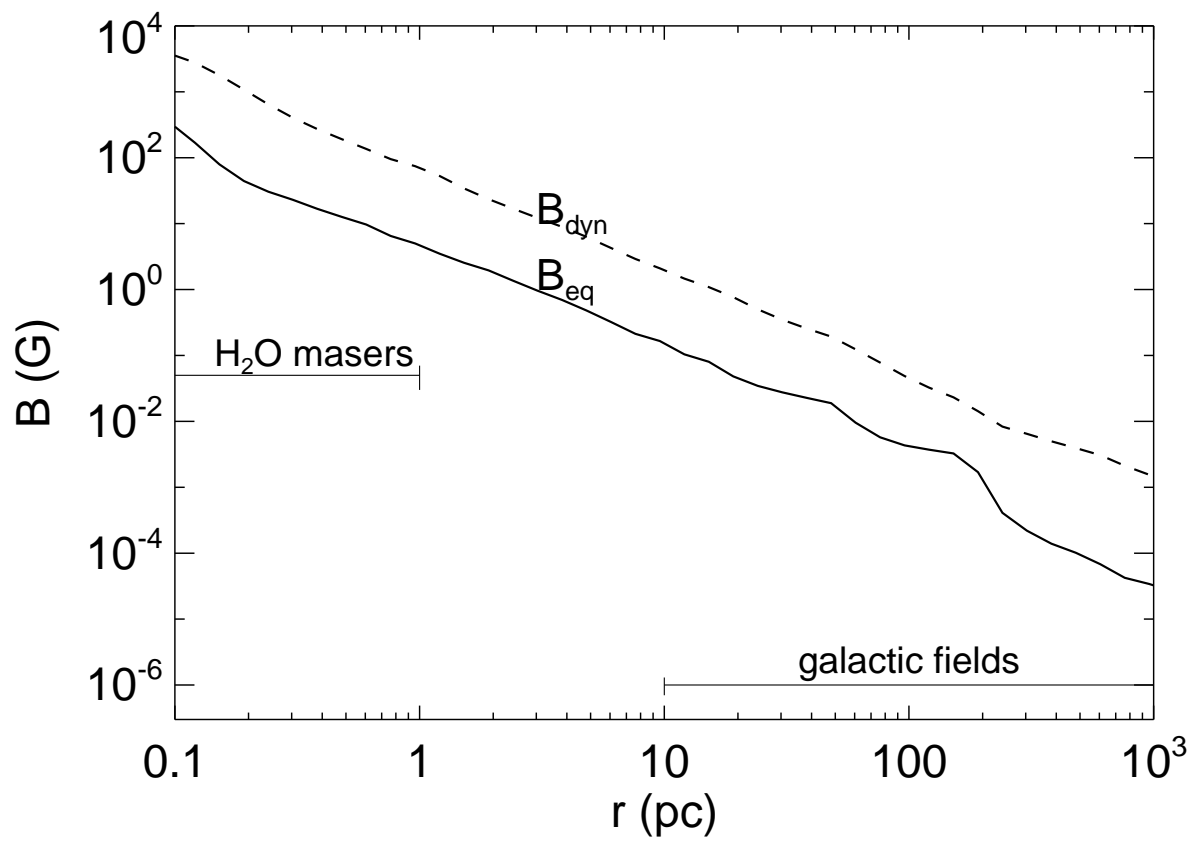

Figure 5.16 Equipartition (solid line) and dynamical (dashed line) magnetic fields needed to influence the gas dynamics in the disk.

Figure 5.16 shows estimates of $B_{\text {eq }}$ and $B_{\text {dyn }}$ in order to demonstrate how high the magnetic field would need to be to significantly influence gas dynamics in the simulated galaxy. For most of the galactic disk, the above estimates for the magnetic field are far higher than the few $\mu \mathrm{G}$ fields observed in real galaxies (e.g. Zweibel \& Heiles, 1997; Beck, 2001). Even in the subparsec region, where water maser observations indicate stronger fields of a few tens of $\mathrm{mG}$ (e.g. Modjaz et al., 2005; Vlemmings et al., 2007), the magnetic field is still too low to affect the gas dynamics in the model galaxy. We therefore conclude that magnetic fields, which are not included in our simulations, will have a negligible effect on the dynamical state of this disk unless their strength greatly exceeds observational measurements. 


\subsection{Discussion and Conclusions}

We have used a large dynamic range cosmological simulation to study gas dynamics in the circumnuclear disk of a typical-mass galaxy at $z \approx 4$ (evolving into an $L_{*}$ galaxy at $z=0$ ), resolving the distribution of matter from megaparsec scales all the way down to subparsec scales (with 20 levels of refinement). The simulation reveals a cold, fully molecular, self-gravitating, and turbulent rotationally supported gas disk, which is globally unstable but locally stable.

The global instability, operating on a range of scales comparable to the size of the system, generates turbulence down to the smallest scale resolved in the simulation. On small scales, the turbulence supports most of the disk against gravitational fragmentation and collapse. The disk, therefore, remains locally stable and reaches a quasi-stationary state. In that state, global instability drives barlike and spiral wave like structures on timescales of the order of 100,000 yr at 100 pc scales (and on proportionally shorter timescales at smaller radii), but on a 500,000 yr timescale the structure of the disk remains quasi-steady.

A combination of gravitational and viscous torques driven by the global instability of the disk drives the outward transport of angular momentum and the inward flow of gas toward the supermassive black hole on timescales of at least 10 million years, which are too long to follow in a single zoom-in episode of the simulation. Thus, we only capture a single snapshot in the cosmological life of the supermassive black hole. In follow-up work, we plan to consider several snapshots taken at different cosmological times in order to describe the system on longer timescales.

The disk develops a power-law gas density profile with a well defined slope

of $-8 / 3$ in spherically-averaged volume density, which is maintained over the duration of the high-resolution simulation. The precise value of the slope of the 
density profile does not straightforwardly arise from the analytic description of the matter transport given in $§ 5.2 .2$, and its origins remain puzzling. It is possible that the behavior of the torques dictates the scaling of the density profile. However, the relationship between the gravitational and viscous torques and the details of their respective roles in angular momentum transport are intricate and require further investigation before an interpretation of the density slope can be offered.

The dynamical state of the disk that we find in our simulation appears to be consistent with the results of previous simulations of isolated circumnuclear disks in galaxies, which find a turbulent, multi-phase ISM (Fukuda et al., 2000; Wada, 2001; Wada \& Norman, 2001; Escala, 2007). A distinctive feature of our approach is that we follow the dynamics of the circumnuclear disk within cosmological simulations. While most of the volume of the simulation evolves little on timescales relevant for the dynamics of the circumnuclear disk, cosmological scales provide realistic boundary conditions for the dynamics on subkiloparsec scales. Since we find that the circumnuclear disk rapidly reaches a quasi-stationary state, its evolution is entirely governed by the boundary conditions. For the same reason, the fact that the cosmological simulation that was used for the initial conditions did not resolve the scale of the circumnuclear disk does not compromise our results; the quasi-stationary state of the disk does not depend on the initial conditions, and so the lack of power on scales below about 100 pc in the initial cosmological simulation is not important.

The adopted approach of this work is comparable to the recent study by Mayer et al. (2007), who used a cosmological simulation to model the coalescence of two supermassive black holes. While the detailed treatment of gas physics and the scientific questions answered by the two studies are different, many of our results are consistent with those of Mayer et al. (2007). For example, they find a similar slope for the spherically averaged density profile, although they do not 
elaborate on the physical mechanisms for the formation of this profile.

The local stability of the disk, supported by highly supersonic turbulence, implies that the disk is capable of continuously feeding a central black hole, uninterrupted by catastrophic bursts of star formation, which could have consumed the available fuel were the disk locally unstable. This interesting dynamical state of the disk provides a potential solution to the problem of how AGN fueling is maintained by self-gravitating gas disks (for a related discussion, see e.g. Shlosman \& Begelman, 1989; Rice et al., 2005; Nayakshin \& King, 2007).

The circumnuclear disk in the simulation extends all the way to the maximally resolved scale of $\approx 0.1 \mathrm{pc}$, which corresponds to the outer part of the black hole accretion disk. We find no toroidal structures on several parsec scales, which are commonly inferred to exist around AGNs. A possible reason that the disk does not form an AGN torus on the appropriate scales (if indeed it should) is the limitation imposed by our implementation of gas cooling in the code. The ART code, like all existing cosmological codes, assumes that the cooling radiation from cosmic gas escapes freely into the IGM. This assumption breaks down at the densities and temperatures reached by the simulation in the inner $10 \mathrm{pc}$. At this scale the disk becomes optically thick to its own cooling radiation from dust and molecular hydrogen. In that regime the disk may heat up and acquire a substantial amount of pressure support, which may result in a puffier, more toroidal configuration for the inner several parsecs of the disk. A consistent treatment of a putative AGN torus will require simulations that incorporate optically thick cooling. 


\section{Chapter 6}

\section{MATTER TRANSPORT IN THE HOST GALAXY OF A SUPERMASSIVE BLACK HOLE}

In the present chapter, we use the simulations and techniques described in detail in Chapters 4 and 5 to study the evolution of the circumnuclear disk of a SMBH host galaxy. Whereas Chapter 5 focused on the structure and dynamics of the circumnuclear disk, emphasis in the present chapter is placed on the temporal evolution. In $\S 6.1$, we briefly summarize the details of the simulations. The results are given in $\S 6.2$, including an analysis of the mass accretion rate and the angular momentum of the gas in the circumnuclear region of the galaxy. Finally, we discuss the results in $§ 6.3$.

\subsection{SIMULATION}

The simulations presented here were run using the Adaptive Refinement Tree (ART) code (Kravtsov et al., 1997; Kravtsov, 1999; Kravtsov et al., 2002). The code follows gas hydrodynamics on an adaptive mesh, and includes dark matter and stellar particles. The gas cooling and heating rates are tabulated as functions of density, temperature, metallicity, and redshift over the temperature range $10^{2}<T<10^{9} \mathrm{~K}$ using CLOUDY (Ferland et al., 1998), which accounts for the metallicity of the gas, and formation of molecular hydrogen and cosmic dust. Presently, only the cosmological portion of the simulation (before zooming- 
in) includes radiative transfer and feedback and enrichment from stars. Stellar particles are formed in cells with appropriate densities and temperatures at an efficiency which matches observed star formation rates on kiloparsec scales (Kennicutt, 1998) and 100pc scales (e.g. Young et al., 1996; Wong \& Blitz, 2002).

A complete description of the zoom-in method is given in Chapter 4, however here we provide a brief overview. Beginning with a cosmological simulation with a maximum resolution of $\approx 180 \mathrm{pc}$ (comoving), the resolution is slowly increased one refinement level at a time, reaching a quasi-stationary state on each level before increasing to the next level. The final, maximum resolution is $\approx 0.089 \mathrm{pc}$ (comoving), corresponding to 20 levels of refinement. After reaching the maximum resolution, a fraction of the gas in the center of the galaxy is replaced with a black hole particle of equal mass and momentum. The simulation continues to evolve with the maximum resolution for several hundred thousand years, but the duration of the simulation varies with redshift. At lower redshifts the increased mass of the galaxy results in more high density cells requiring high resolution. Following a larger portion of the simulation with finer resolution is more computationally expensive, therefore the lower redshift simulations take longer to evolve. As a result a redshift 3 simulation studied here has only evolved half as long in physical time as a redshift 4 simulation.

Most of the following analysis is applied to the short-term evolution of the galaxy $\left(10^{5}-10^{6} \mathrm{yr}\right)$ during a zoom-in episode at $z=4$ containing a $3 \times 10^{7} M_{\odot}$ SMBH particle (considered the fiducial run). Additional simulations were performed, including zoom-in episodes at other redshifts with the same black hole mass $(z=3$ and 6$)$ and with different black hole masses $\left(9 \times 10^{7} M_{\odot}\right.$ and $\left.3 \times 10^{8} M_{\odot}\right)$ at $z=4$. Table 6.1 summarizes the details of the simulations performed, including redshift, galaxy mass, black hole mass, and the duration of each run in physical time. 
Table 6.1. Summary of each simulation run

\begin{tabular}{lcccc}
\hline \hline Run & $z$ & $M_{\text {gal }}\left(10^{10} M_{\odot}\right)$ & $M_{\mathrm{BH}}\left(10^{7} M_{\odot}\right)$ & Length of Run $(\mathrm{yr})$ \\
\hline $\mathrm{A} 120$ & 3 & 18 & 3 & 550,000 \\
$\mathrm{C} 120^{*}$ & 4 & 13 & 3 & $1,100,000$ \\
$\mathrm{D} 120$ & 6 & 5.5 & 3 & $1,500,000$ \\
$\mathrm{C} 220$ & 4 & 13 & 9 & 700,000 \\
$\mathrm{C} 320$ & 4 & 13 & 30 & 350,000 \\
\hline
\end{tabular}

*fiducial run

It is expected that the mass of the black hole should continue to grow with decreasing redshift. Therefore, the different redshift simulations do not provide the growth history of a single galaxy because they each contain the same mass black hole particle. Instead, they represent the distinct growth histories of similar disk galaxies.

The simulation C120 was chosen for the fiducial run because $z=4$ is a relatively quiescent stage in the growth of the host galaxy. At this redshift, the main galaxy of the simulation has not undergone a large mass ratio merger in $\approx 600$ million years, but the galaxy is still actively growing. In the simulation D120 $(z=6)$, the galaxy is less relaxed, as it is undergoing a 4:1 merger at that time.

\subsection{RESULTS}

Chapter 5 gives the details of the spatial structure of the circumnuclear region for the fiducial run. Each of the simulations described here show similar structure: a cold, rotationally dominated, self-gravitating gas disk develops in the circumnuclear region of the galaxy (inside $\sim 100 \mathrm{pc}$ ), following a power-law density distribution with a slope of $-8 / 3$ in volume density. The disk is globally unsta- 
ble, leading to the development of waves and instabilities which drive turbulence on a range of scales, injecting energy through shocks. The turbulence is highly supersonic and acts to maintain local stability. The transient features in the disk, caused by spiral waves and global instabilities, slowly allow angular momentum transport, driving gas inward toward the center of the galaxy. In this section, we explore the timescales important for transport of this gas, and the behavior over time of the rate of transport and the angular momentum of the gas throughout the circumnuclear disk.

\subsubsection{Mass of the Disk and the Rate of Transport}

Figure 6.1 shows the mean gas mass, $\bar{M}$, interior to radius, $r$, for each simulation (averaged over the duration of the simulation). The radius $r$ is measured with respect to the center of the galaxy, given by the position of the black hole particle. A comparison of the average gas mass in each zoom-in episode shows the growth of the galaxy over cosmological times. However, the instantaneous profiles at individual times throughout the zoom-in part of the simulation reveal the transient nature of the circumnuclear disk. As the globally unstable disk forms transient structures on a range of scales, the interior gas mass on measured on scales $\leq 100$ pc can vary significantly. Figure 6.2 shows the amplitude of mass fluctuations, $M(t) / \bar{M}$ at four different radii (ranging from 0.1 to $100 \mathrm{pc}$ ) over time for each of the simulations. The figure gives an indication of the turbulent nature of the gas in the circumnuclear disk of the galaxy.

Around 550,000yr in the $z=4$ simulation, a clump of gas (formed as a result of the global instabilities in the disk) moves into the center, temporarily creating a gravitational disturbance which displaces the black hole particle for $\approx 50,000$ yr. This is, perhaps, a numerical effect resulting from the somewhat arbitrarily chosen black hole particle mass. The black hole soon resettles into the bottom 


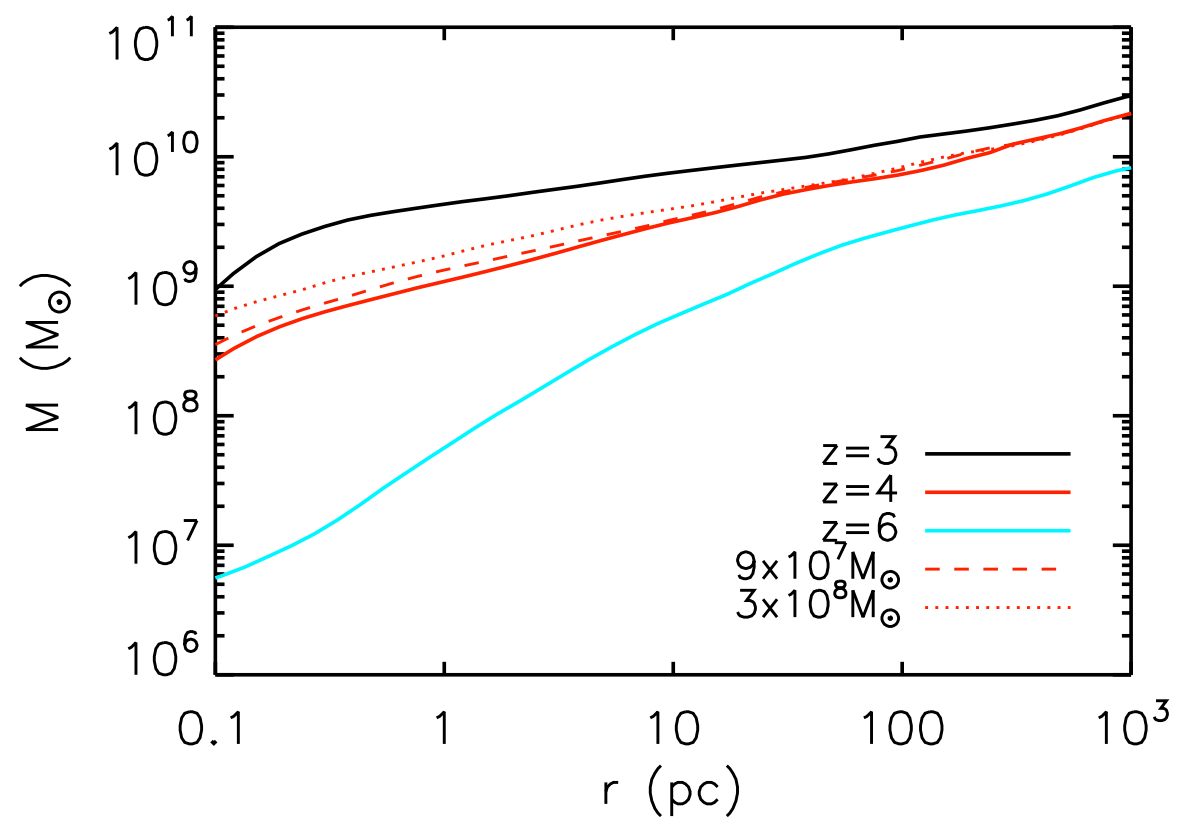

Figure 6.1 The time-averaged interior gas mass as a function of radius for each simulation.

of the potential well of the disk, but because the interior mass is measured with respect to the black hole particle, the interior mass measurements are temporarily affected by the black hole's motion. Therefore, the interior gas mass in Figure 6.2 has been interpolated over the duration of the black hole displacement. We are investigating the cause of the displacement, in order to better understand how the properties of the massive clump of gas might be significant for the angular momentum of the disk, which will be discussed further in $\$ 6.2 .2$.

As Figure 6.2 shows, it is difficult to determine a characteristic accretion rate from any one individual snapshot of the circumnuclear region, given the amount of fluctuation in the gas mass over the timescale of the simulations. In order to estimate the accretion rates, it is necessary to first understand how the mass fluctuations depend on timescale. The Fourier transform of the interior gas mass is therefore a useful quantity for studying the frequency dependence. The mass (and 


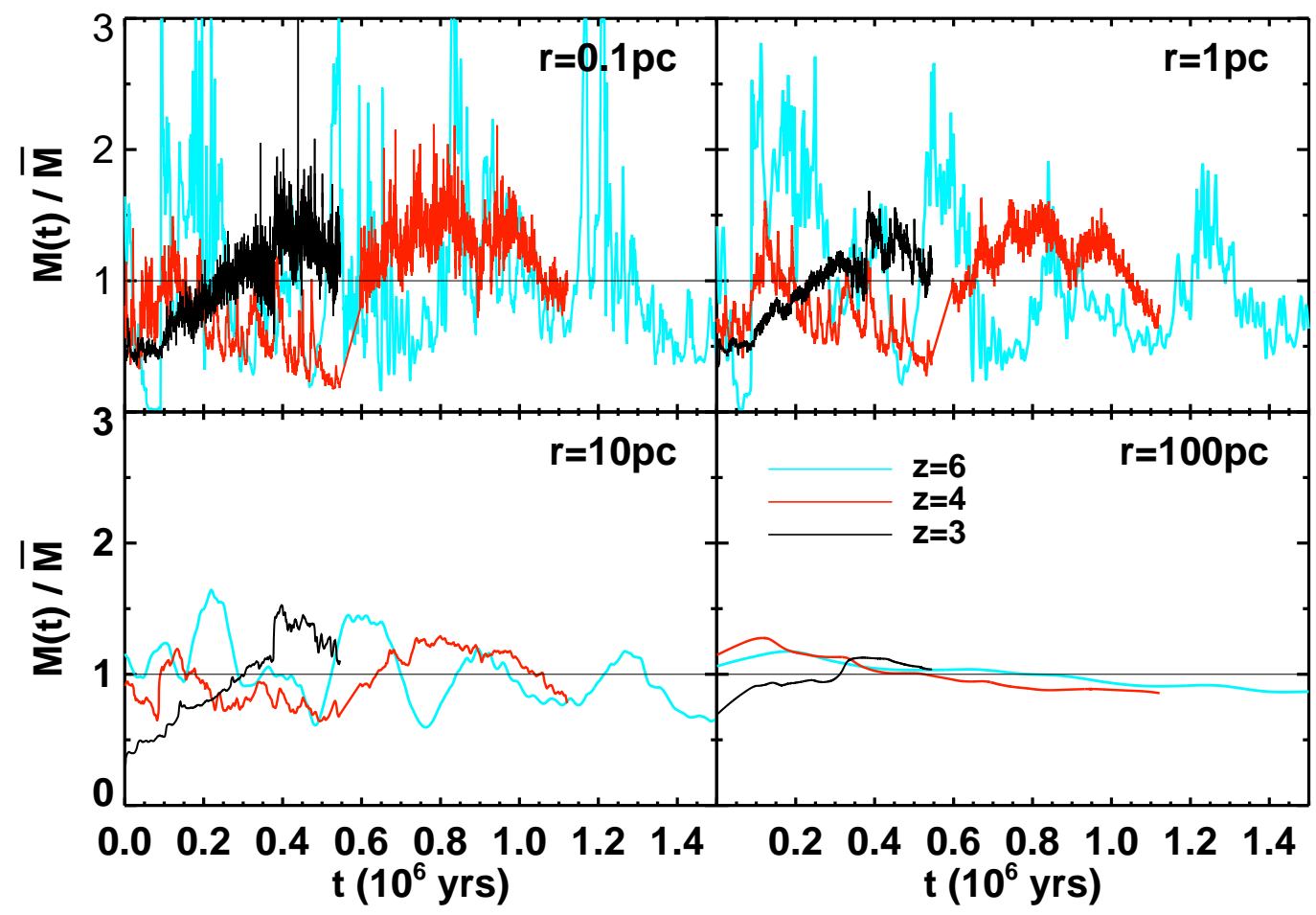

Figure 6.2 Ratio of the interior gas mass to the time-averaged value at redshifts 3, 4 , and 6 for 4 different radii. The solid line shows $M=\bar{M}$ for comparison. (Fig. 6.1 shows the $\bar{M}$ for each simulation).

all other physical quantities) are sampled every level 6 time step ${ }^{1}$. The sampling frequency is somewhat arbitrary, but is such that the entire circumnuclear region evolves between steps (since the whole region is resolved with at least 9 levels of refinement). During the simulation, the physical time step is recalculated after every level 0 step, and depends on the dynamics of the disk. Therefore the physical length of the sampling interval (a level 6 step), varies throughout the duration of the simulation. The Lomb-normalized periodogram (see Lomb, 1976; Press et al., 1992, Numerical Recipes, Sec. 13.8), or the spectral power normalized by the variance of a dataset, is useful for characterizing the behavior of unevenly sampled data, such as the uneven time steps here. The normalized spectral power

\footnotetext{
1 Time steps forward in the simulation so that for each step on level $l$, the simulation has taken $2^{l_{\max }-l}$ time steps at the maximum resolution level, $l_{\max }$.
} 
is given by

$$
P_{\mathrm{N}}(\omega)=\frac{\left[\sum_{j=1}^{N}\left(M_{j}-\bar{M}\right) \cos \omega\left(t_{j}-\tau\right)\right]^{2}}{2 \sigma^{2} \sum_{j=1}^{N} \cos ^{2} \omega\left(t_{j}-\tau\right)}+\frac{\left[\sum_{j=1}^{N}\left(M_{j}-\bar{M}\right) \sin \omega\left(t_{j}-\tau\right)\right]^{2}}{2 \sigma^{2} \sum_{j=1}^{N} \sin ^{2} \omega\left(t_{j}-\tau\right)}
$$

summed over $N$ data points, where $\sigma^{2}$ is the variance in the mass. The quantity $\tau$, defined by

$$
\tan (2 \omega \tau)=\frac{\sum_{j=1}^{N} \sin 2 \omega t_{j}}{\sum_{j=1}^{N} \cos 2 \omega t_{j}},
$$

is introduced so the spectral power is independent of shifting the data in time. Although the Lomb-normalized periodogram is not directly computed from the Fourier transform of the data, we use it as an estimate for the Fourier transform of the interior gas mass. Then Equation 6.1 gives an estimate of the mass as a function of angular frequency $\omega$ since

$$
\begin{aligned}
M_{\omega} & =\frac{1}{N} \sum_{j=1}^{N} M\left(t_{j}\right) e^{i \omega t_{j}} \\
& \approx \sqrt{\frac{P_{\mathrm{N}} \sigma^{2}}{N}}
\end{aligned}
$$

The Fourier transform of the mass follows a power-law with slope -1 in each of the simulations, showing no substantial departure down to frequencies comparable to the rotational period of the disk. The characteristic accretion rate through radius $r$ as a function of frequency is then

$$
\dot{M}_{\omega}=i \omega M_{\omega}
$$




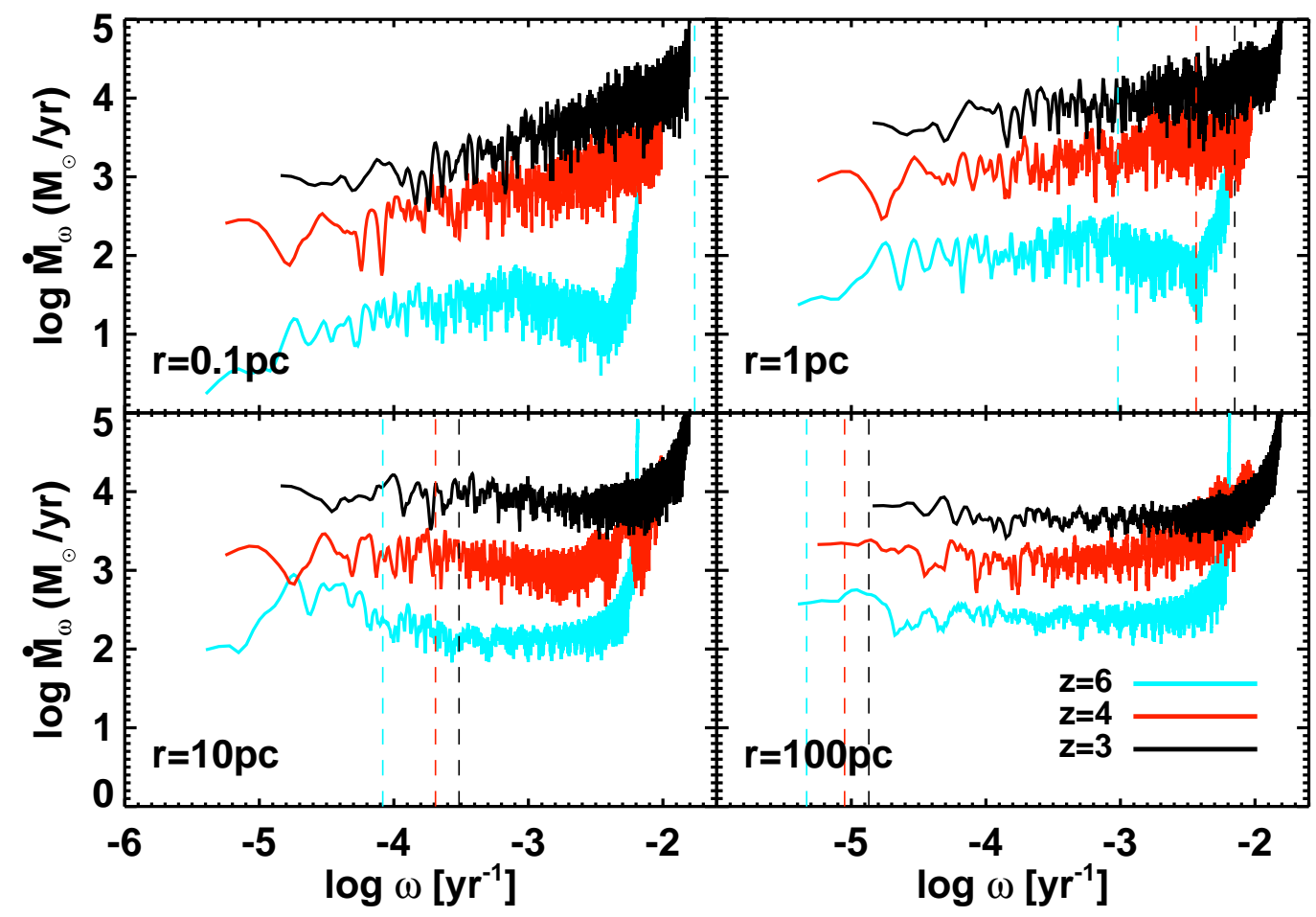

Figure 6.3 The Fourier transform of the mass accretion rate, $\dot{M}(\omega)$, as a function of frequency for three different redshifts (smoothed over 5 neighboring points). The vertical lines correspond to the mean angular velocity in each simulation, at the scales given in each panel.

Strictly speaking, the "accretion rate" given by Equation 6.4 does not describe the net inward transport of gas, but rather it gives typical values of the mass flux of gas flowing into and out of a given region on a timescale $2 \pi / \omega$. The flat slope in the Fourier transform of the accretion rate indicates that it is independent of frequency, or that accretion is a stochastic process with no preferred timescale. Simulations of self-gravitating disks have found behavior similar to our own results, with instabilities driving turbulence that leads to intermittent fueling episodes lasting $10^{4}-10^{5} \mathrm{yr}$ (Wada et al., 2002; Wada, 2004).

The absolute value of the accretion rate given by Equation 6.4 is shown as a function of frequency in Figures 6.3 and 6.4. The data are smoothed over 5 neighboring points to reduce the noise so that the different curves are readily 




Figure 6.4 The Fourier transform of the mass accretion rate, $\dot{M}(\omega)$, as a function of frequency for three different black hole masses at $z=4$ (smoothed over 5 neighboring points). The vertical lines correspond to the mean angular velocity in each simulation, at the scales given in each panel.

distinguishable. The mass flux is larger for decreasing redshift, because the galaxy itself is more massive, but the flux follows the same stochastic behavior at different epochs (Fig. 6.3), as well as for different black hole masses (Fig. 6.4). The mass flux appears slightly higher for larger black hole masses, however when the data is unsmoothed, the differences are less pronounced. The vertical lines in each panel correspond to the angular velocity of each simulation at a given scale. The fluctuations do not appear to be correlated with the orbital period for each scale.

A simple way to estimate the net accretion rate over the timescale of each simulation, albeit roughly, is to measure the difference in mass at each scale between the beginning and the end of each run. However, even after evolving for a million years, as in the $z=4$ and $z=6$ simulations, more mass has flowed 
outward at some scales than has flowed inward (this can be seen in Fig. 6.2). Since the Fourier transform of the accretion rate remains flat down to frequencies corresponding to the timescale of the simulation, and is therefore still stochastic, it is perhaps not surprising that an average accretion rate cannot be estimated this way.

The complicated dynamics of the gas in the circumnuclear region and the stochastic nature of accretion make it difficult to constrain the accretion rate onto the SMBH on short timescales. More detailed studies following the gas for at least several million years are necessary in order to understand the long term effects of such behavior. At present, there is no prescription for the growth of black hole particle in the simulation. An accretion rate might be determined by measuring how much of the gas on the smallest scales is bound to the black hole particle, and then assuming some fraction is accreted. Given the unprecedentedly high resolution of the simulations, such an approximation is worthwhile despite the lack of accretion disk physics in the code. Future studies will incorporate accretion in this or a similar manner, allowing us to explore more thoroughly whether the properties found in the high-resolution portion of our simulations are consistent with the assumptions typically made about accretion within lower resolution simulations (e.g. Bondi accretion).

The accretion rate over cosmological times can be estimated by comparing the mean interior gas mass (the averages shown in Fig. 6.1) for the different redshift simulations. Figure 6.5 shows the mean gas mass, interior to radius $r$ as a function of the age of the universe, $t_{\text {age }}$ ( $\mathrm{a} z=3.5$ run is included in Fig. 6.1, which is absent from the rest of the analysis, but consistent with the results). Once again we emphasize that the different redshift simulations do not necessarily describe different stages of growth of the same galaxy because they each contain the same mass SMBH (rather than a black hole which grows with redshift). However, the 


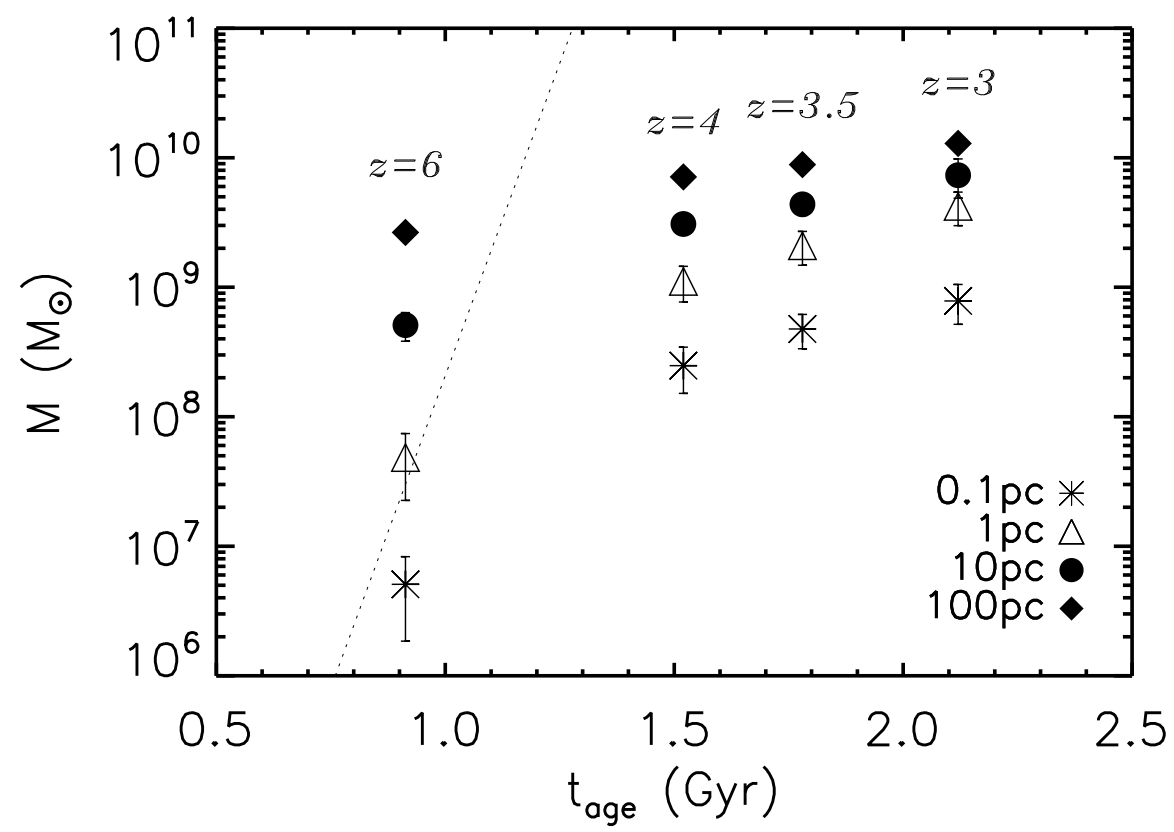

Figure 6.5 Mean gas mass interior to radius $r$ as a function of the age of the universe, $t_{\text {age }}$.

black hole particle itself does not play a large role in the evolution of the galaxy (at least at $z=4$ and 3, where the mass of the black hole is dominated by the gas mass all the way down to the center).

The dotted line in Figure 6.5 shows the mass of a black hole, initially $3 \times$ $10^{7} M_{\odot}$ at $z=6$, if it grows continuously at the Eddington limit according to

$$
M(t)=M_{\mathrm{BH}} \mathrm{e}^{t / t_{\mathrm{S}}},
$$

where $t_{\mathrm{S}}$ is the Salpeter time (Salpeter, 1964), and for a radiative efficiency $\eta=0.1$. If the dynamics of the circumnuclear disk extend all the way down to scales beneath our resolution (which we cannot assume) then the growth rate, which appears to steepen with decreasing scale, may continue to steepen down to smaller scales, approaching the Eddington limit. Therefore, a black hole growing according to Equation 6.5 may grow much more rapidly than the gas disk in the 
circumnuclear region does on the scales we resolve. However, as cautioned in Chapter 5, the results in the central few parsecs should be considered preliminary, because the simulations do not yet include a proper treatment of optically thick cooling. Without accounting for the high column densities of molecular gas and dust in the center of the galaxy, the cooling rates are incorrect, and we cannot accurately describe structures such as the proposed "obscuring torus," which may effect transport in this region. Furthermore, the actual accretion rate onto the black hole is governed by the physics of the accretion disk, also not modeled by our simulations. Nonetheless, the increase in the mass of the circumnuclear gas disk over cosmological times yields enough fuel to build up the mass of the SMBH if the fuel can be efficiently accreted.

\subsubsection{Angular Momentum}

The large fluctuations in the interior gas mass, caused by waves moving through the circumnuclear disk, will stochastically drive individual clumps of molecular gas into the vicinity of the SMBH, ultimately forming an accretion disk whose angular momentum could be randomly oriented with respect to the rest of the circumnuclear disk. If the angular momentum of an individual clump is oriented randomly with respect to the black hole's spin axis, successive accretion events will reduce the spin of the black hole, decreasing its accretion efficiency (King \& Pringle, 2006, 2007; King et al., 2008). It is therefore useful in characterizing accretion to measure the angular momentum of the gas on small scales.

In the simulations here, we do not follow the gas down to scales inside the accretion disk. However, the simulation does model gas on parsec scales, enabling us to resolve the direction of the angular momentum vector, and to provide boundary conditions for simulations of SMBH accretion disks. The angular mo-

mentum vector is measured with respect to the black hole particle's position and 
velocity. During most of the simulation, the black hole particle lies in the densest cell, which coincides with the minimum of the galaxy's gravitational potential. Therefore, the black hole particle typically provides a reasonable estimate of the location of center of the galaxy.

The velocity of the center with respect to the fixed mesh is more difficult to define. Determining the velocity at the center of the galaxy allows a transformation to the rest frame of the galaxy. The velocity of the central cell does not necessarily give an accurate estimate of the motion of the galaxy because of the discreteness of the cell. The black hole velocity may contain a significant rotational component as it moves within the central cell, introducing a small offset to the velocities defined in the rest of the disk. At present, we use the velocity of the black hole particle to define the rest frame of the galaxy, but we plan to explore alternative methods in the future, such as taking the mean of the velocities over several cells in the center.

Figure 6.6 shows a map projection of the direction of the normalized angular momentum vector as it evolves in time at both 100 and 1pc (top and bottom panels, respectively). The $z$-axis of the simulation grid is oriented vertically in the map. The rotation axis of the galaxy on kiloparsec scales points in the $x$ direction, but the disk is slightly warped on smaller scales, so that the axis of the circumnuclear disk is oriented in the $x z$-direction of the grid.

At $100 \mathrm{pc}$, the direction of the angular momentum vector changes slowly, but shows little scatter over the course of the simulation. The angular momentum at the $1 \mathrm{pc}$ scale shows significantly more scatter, which may be consistent with models in which SMBH growth occurs via accretion of clumps of gas with randomly oriented angular momentum axes. The apparent flip in the direction of the angular momentum axis corresponds to the time of the displacement of the black hole particle described in $§ 6.2 .1$. As mentioned above, we are currently 



Figure 6.6 Map showing the direction of the angular momentum vector, $\boldsymbol{L}$ as it changes over time at 100 and $1 \mathrm{pc}$. The colors correspond to the time in the simulation since the introduction of the black hole particle. The $z$-axis of the simulation grid is oriented vertically in the map. At the 100pc scale, the axis of the circumnuclear disk is oriented primarily in $x z$-direction. 
investigating the cause of the displacement and of the change in direction of the angular momentum axis.

\subsection{DISCUSSION AND CONCLUSIONS}

We have followed the transport of gas in the circumnuclear region of a disk galaxy within a cosmological simulation, and we find that there is no preferred timescale for accretion over the course of the $\sim$ million years spanned by the simulations. The interior gas mass at each scale fluctuates throughout the circumnuclear disk, showing negative accretion rates as often as positive. Characterizing the fluctuations as a function of timescale, the flat slope of the Fourier transformed mass accretion rate further illustrates the stochastic nature of accretion in the simulations. The behavior is apparent at each of the three redshifts

explored here: $z=3,4$, and 6 , as well as in $z=4$ simulations containing black holes with larger masses.

The results presented here are perhaps consistent with models suggesting that stochastic accretion of molecular clouds from the circumnuclear region of the galaxy can power low-luminosity AGN (Hopkins \& Hernquist, 2006). This manner of accretion will keep the spin of the black hole low, perhaps explaining the radioquietness of disk galaxies (Berti \& Volonteri, 2008). However, further studies of the angular momentum of the gas in the circumnuclear region are necessary. Presently, we find that the angular momentum vector on the $100 \mathrm{pc}$ scale shows little scatter over time and follows the general orientation of the circumnuclear disk. However, on parsec scales the angular momentum shows more scatter, and can be misaligned with the rest of the circumnuclear disk, perhaps resembling the stochastic fueling scenarios mentioned above.

The main result regarding both the mass accretion rate and the angular momentum of the gas in the circumnuclear region is that the behavior is complex 
and chaotic. This result enforces the need for high-resolution simulations, in order to truly model the complicated dynamics on small scales which ultimately govern accretion onto SMBHs. The first of our immediate goals is to understand the longer term evolution of the stochastic accretion rates (for many millions of years, which approaches the proposed lifetimes of AGNs). The second goal is to understand the cause of the displacement of the black hole particle in the simulations, in order to better understand the dynamics on the smallest scales. Additionally, our plans to implement an approximation for optically thick cooling in the ART code will allow us to examine the role of the proposed AGN obscuring torus on the mass transport in the central few parsecs. Modeling this region correctly is essential if we are to understand how gas is transported all the way down to the accretion disk of the SMBH. 


\section{Chapter 7}

\section{CONCLUSIONS AND THE DIRECTION OF FUTURE RESEARCH}

\subsection{Summary of Results}

After combining a simple semi-analytic model of the expansion of AGN outflows into the IGM with a simulated dark matter density distribution, we conclude that outflows with kinetic fractions higher than $\sim 1 \%$ would fill the entire IGM by $z=2$. Therefore, the observed filling fraction of the Ly $\alpha$ forest at different redshifts constrains the kinetic fraction of outflows to be $\leq 1 \%$, because a lower kinetic fraction reduces the cosmological volume filled with outflows. The filling fraction of outflows also depends on bias in the distribution of AGN (a population

more heavily biased toward high-density regions lowers the filling fraction) and on AGN lifetimes (longer lifetimes lower the filling fraction as well).

The outflow model of Chapter 2 shows that AGN feedback is potentially energetic enough to impact the environment out to cosmological scales by redistributing baryons from small scales to large scales. Though baryons make a small contribution to the matter density of the universe, changing their distribution with outflows may affect the interpretation of cosmological parameters derived from weak lensing results, which depend on the entire matter distribution, without distinguishing between baryons and dark matter. If AGN outflows play a role, they will distribute baryons more evenly throughout the universe, decreasing their contribution to the matter power spectrum. However, the density perturbations 
imposed by AGN outflows on non-linear scales may have the effect of increasing the power on large scales, because AGN themselves are clustered. From the analysis presented in Chapter 3, it is unclear which of these effects is likely to be more prominent, warranting detailed hydrodynamical simulations in order to study the effects of AGN outflows over a range of scales.

The eventual impact of AGN feedback depends initially on the intricate environments where AGN are first formed. Since AGN are driven by accreting SMBHs, it is necessary to model the complex galactic environments which fuel SMBHs. With a cosmological AMR simulation containing gas hydrodynamics, a detailed treatment of baryonic physics, stars, dark matter and a SMBH particle, we have studied the dynamics of gas within the circumnuclear region of a Milky Way-progenitor type galaxy. This thesis represents the first stage in building a simulation which can realistically model the fueling of a $\mathrm{SMBH}$ in a cosmological context.

The simulations here have revealed a cold, self-gravitating, molecular gas disk which is globally unstable. The chaotic formation and re-formation of waves and instabilities in the disk drives turbulence which acts to maintain stability on small scales. The stability on small scales prevents the disk from collapsing catastrophically and turning completely into stars, leaving fuel for the SMBH. Gravitational forces generate a torque, which is the dominant mechanism of angular momentum transport in the disk. A viscous torque arising from the supersonic turbulence in the disk may also contribute to the transport of angular momentum, although it is not yet clear how big a role viscous torques play in the dynamics of the circumnuclear region.

The chaotic conditions within the circumnuclear gas disk make it difficult to characterize the accretion rate in the disk. The interior gas mass fluctuates significantly so that the accretion rate is as often negative as it is positive, and 
is best characterized by a power spectrum. The power spectrum is mostly flat, meaning that the accretion is a stochastic process, with no preferred timescale. It is not immediately clear from the results how this behavior relates the cosmological evolution of the galaxy to the growth of the SMBH. However, the complicated picture suggests that high-resolution simulations of the type presented here are essential for understanding the accretion.

\subsection{Future Work with the ART Code}

The numerical techniques and methodology adopted in Chapters 4-6 are well suited for addressing a number of interesting problems related to SMBH growth. In addition to some follow-up analysis of issues that have arisen during the previous simulations, here are a few of the main projects we plan to address. This list is by no means exhaustive, given the richness of the topic of SMBH and galaxy co-evolution and the capabilities of current numerical techniques.

\section{Follow-up Analysis}

The complex behavior uncovered by these simulations in the circumnuclear disk warrants further investigation. In the immediate future we will investigate the cause of the black hole displacement occurring in some of the simulations, and the properties of the infalling gas clump which led to the displacement (in particular, its angular momentum). Additionally, we will explore the long term effects of stochastic accretion and see if the behavior extends to $10^{7}$ or $10^{8} \mathrm{yr}$ timescales.

\section{Optically Thick Cooling}

Presently, we are implementing an approximation for optically thick cooling in the code and will study the effects on structures in the central few parsecs. 
In this region, at column densities in excess of $\sim 10^{26}$ and $\sim 10^{27} \mathrm{~cm}^{-2}$, both $\mathrm{H}_{2}$ and dust, respectively, become optically thick to their own cooling radiation. The approximation we are adopting reduces the cooling rate by a factor of $\mathrm{e}^{-\tau}$, where $\tau$ is proportional to a length scale calculated at each point from the local density gradient. This approximation appears to work well over more than 5 orders of magnitude in column density. Adjusting the cooling in this manner will be essential for studying structures, such as the so-called "obscuring torus," which are expected to play an important role in an AGN.

\section{AGN Feedback}

The set of simulations presented here do not include feedback from the $\mathrm{SMBH}$, and in the near future we plan to implement a prescription for both mechanical and radiative feedback (in the form of a line-driven wind). Mechanical feedback can be implemented straightforwardly, as an injection of kinetic energy into the gas surrounding the SMBH. Such a technique is already well developed for modeling supernova feedback in cosmological simulations (see e.g. Springel \& Hernquist, 2003; Springel, 2005; Springel et al., 2005; Oppenheimer \& Davé, 2006; Stinson et al., 2006; Governato et al., 2007). The high-resolution of our simulations will allow us to identify the axis of the accretion disk, and to explore the effects of jets with varying inclination. Line-driven winds are less straightforwardly implemented, but can be modeled by using the Sobolev approximation to determine the optical depth of the winds. With simulations of feedback in hand,

we can study the interaction of AGN feedback with the circumnuclear region of the galaxy, and begin to address more thoroughly the impact of feedback on larger scales (see Chaps. 2 and 3).

\section{SMBH Mergers}


The studies presented here have focused primarily on the fueling of the SMBH in the central galaxy. In the $z=6$ run (D120), the main galaxy was in the process of merging with a galaxy $25 \%$ its size, but our focus was on the larger of the two galaxies. In future work, we plan to follow a galaxy merger between the main galaxy and a smaller satellite also containing a SMBH. As the galaxies merge, the smaller galaxy's SMBH will move toward the center of the main galaxy and into the high resolution region. We can then follow the SMBH through the circumnuclear region, down to subparsec scales, and assess the role of gas and stars in removing angular momentum from the merging pair. 


\section{Bibliography}

Abel, T., Bryan, G. L., \& Norman, M. L. 2002, Science, 295, 93

Annis, J., Bridle, S., Castander, F. J., Evrard, A. E., Fosalba, P., Frieman, J. A., Gaztanaga, E., Jain, B., Kravtsov, A. V., Lahav, O., Lin, H., Mohr, J., Stebbins, A., Walker, T. P., Wechsler, R. H., Weinberg, D. H., \& Weller, J. 2005, Constraining Dark Energy with the Dark Energy Survey: Theoretical Challenges

Balbus, S. A., \& Hawley, J. F. 1998, Reviews of Modern Physics, 70, 1

Barger, A. J., Cowie, L. L., Mushotzky, R. F., Yang, Y., Wang, W.-H., Steffen, A. T., \& Capak, P. 2005, AJ, 129, 578

Barnes, J. E., \& Hernquist, L. 1992, ARA\&A, 30, 705

Beck, R. 2001, Space Science Reviews, 99, 243

Begelman, M. C., Blandford, R. D., \& Rees, M. J. 1984, Reviews of Modern Physics, 56, 255

Begelman, M. C., Volonteri, M., \& Rees, M. J. 2006, MNRAS, 370, 289

Benson, A. J., \& Madau, P. 2003, MNRAS, 344, 835

Berti, E., \& Volonteri, M. 2008, ArXiv e-prints, 802

Bondi, H. 1952, MNRAS, 112, 195

Bondi, H., \& Hoyle, F. 1944, MNRAS, 104, 273

Boone, F., Baker, A. J., Schinnerer, E., Combes, F. ., García-Burillo, S., Neri, R., Hunt, L. K., Léon, S., Krips, M., Tacconi, L. J., \& Eckart, A. 2007, A\&A, 471, 113

Bottema, R. 2003, MNRAS, 344, 358

Boyle, B. J., Shanks, T., Croom, S. M., Smith, R. J., Miller, L., Loaring, N., \& Heymans, C. 2000, MNRAS, 317, 1014 
Bromm, V., \& Loeb, A. 2003, ApJ, 596, 34

Bromm, V., Yoshida, N., \& Hernquist, L. 2003, ApJ, 596, L135

Brotherton, M. S., van Breugel, W., Smith, R. J., Boyle, B. J., Shanks, T., Croom, S. M., Miller, L., \& Becker, R. H. 1998, ApJ, 505, L7+

Brüggen, M., \& Kaiser, C. R. 2002, Nature, 418, 301

Carr, B. J., Bond, J. R., \& Arnett, W. D. 1984, ApJ, 277, 445

Chiu, W. A., \& Ostriker, J. P. 2000, ApJ, 534, 507

Christodoulou, D. M., Shlosman, I., \& Tohline, J. E. 1995a, ApJ, 443, 551

—. 1995b, ApJ, 443, 563

Colberg, J. M., \& Di Matteo, T. 2008, ArXiv e-prints, 804

Crampin, D. J., \& Hoyle, F. 1964, ApJ, 140, 99

Crenshaw, D. M., Kraemer, S. B., Boggess, A., Maran, S. P., Mushotzky, R. F., \& Wu, C.-C. 1999, ApJ, 516, 750

Cristiani, S., Alexander, D. M., Bauer, F., Brandt, W. N., Chatzichristou, E. T., Fontanot, F., Grazian, A., Koekemoer, A., Lucas, R. A., Mao, J., Monaco, P., Nonino, M., Padovani, P., Stern, D., Tozzi, P., Treister, E., Urry, C. M., \& Vanzella, E. 2005, in Multiwavelength Mapping of Galaxy Formation and Evolution, ed. A. Renzini \& R. Bender, 145-+

Cristiani, S., Alexander, D. M., Bauer, F., Brandt, W. N., Chatzichristou, E. T., Fontanot, F., Grazian, A., Koekemoer, A., Lucas, R. A., Monaco, P., Nonino, M., Padovani, P., Stern, D., Tozzi, P., Treister, E., Urry, C. M., \& Vanzella, E. 2004, ApJ, 600, L119

Croom, S. M., Boyle, B. J., Shanks, T., Smith, R. J., Miller, L., Outram, P. J., Loaring, N. S., Hoyle, F., \& da Ângela, J. 2005, MNRAS, 356, 415

Davé, R., Hernquist, L., Katz, N., \& Weinberg, D. H. 1999, ApJ, 511, 521

de Kool, M., Arav, N., Becker, R. H., Gregg, M. D., White, R. L., LaurentMuehleisen, S. A., Price, T., \& Korista, K. T. 2001, ApJ, 548, 609

Di Matteo, T., Colberg, J., Springel, V., Hernquist, L., \& Sijacki, D. 2007, ArXiv e-prints, 705

Di Matteo, T., Springel, V., \& Hernquist, L. 2005, Nature, 433, 604

Draine, B. T., \& Lee, H. M. 1984, ApJ, 285, 89 
Duncan, R. C., Ostriker, J. P., \& Bajtlik, S. 1989, ApJ, 345, 39

Ebisuzaki, T., Makino, J., Tsuru, T. G., Funato, Y., Portegies Zwart, S., Hut, P., McMillan, S., Matsushita, S., Matsumoto, H., \& Kawabe, R. 2001, ApJ, 562, L19

Efstathiou, G., Lake, G., \& Negroponte, J. 1982, MNRAS, 199, 1069

Eisenstein, D. J., \& Loeb, A. 1995, ApJ, 443, 11

Elitzur, M., \& Shlosman, I. 2006, ApJ, 648, L101

Elvis, M., Wilkes, B. J., McDowell, J. C., Green, R. F., Bechtold, J., Willner, S. P., Oey, M. S., Polomski, E., \& Cutri, R. 1994, ApJS, 95, 1

Escala, A. 2007, ArXiv e-prints, 705

Fan, X., Narayanan, V. K., Lupton, R. H., Strauss, M. A., Knapp, G. R., Becker, R. H., White, R. L., Pentericci, L., Leggett, S. K., Haiman, Z., Gunn, J. E., Ivezić, Ž., Schneider, D. P., Anderson, S. F., Brinkmann, J., Bahcall, N. A., Connolly, A. J., Csabai, I., Doi, M., Fukugita, M., Geballe, T., Grebel, E. K., Harbeck, D., Hennessy, G., Lamb, D. Q., Miknaitis, G., Munn, J. A., Nichol, R., Okamura, S., Pier, J. R., Prada, F., Richards, G. T., Szalay, A., \& York, D. G. 2001a, AJ, 122, 2833

Fan, X., Strauss, M. A., Schneider, D. P., Becker, R. H., White, R. L., Haiman, Z., Gregg, M., Pentericci, L., Grebel, E. K., Narayanan, V. K., Loh, Y.-S., Richards, G. T., Gunn, J. E., Lupton, R. H., Knapp, G. R., Ivezić, Ž., Brandt, W. N., Collinge, M., Hao, L., Harbeck, D., Prada, F., Schaye, J., Strateva, I., Zakamska, N., Anderson, S., Brinkmann, J., Bahcall, N. A., Lamb, D. Q., Okamura, S., Szalay, A., \& York, D. G. 2003, AJ, 125, 1649

Fan, X., Strauss, M. A., Schneider, D. P., Gunn, J. E., Lupton, R. H., Becker, R. H., Davis, M., Newman, J. A., Richards, G. T., White, R. L., Anderson, Jr., J. E., Annis, J., Bahcall, N. A., Brunner, R. J., Csabai, I., Hennessy, G. S., Hindsley, R. B., Fukugita, M., Kunszt, P. Z., Ivezić, Ž., Knapp, G. R., McKay, T. A., Munn, J. A., Pier, J. R., Szalay, A. S., \& York, D. G. 2001b, AJ, 121, 54

Ferland, G. J., Korista, K. T., Verner, D. A., Ferguson, J. W., Kingdon, J. B., \& Verner, E. M. 1998, PASP, 110, 761

Ferrarese, L., \& Merritt, D. 2000, ApJ, 539, L9

Fujita, A., Mac Low, M.-M., Ferrara, A., \& Meiksin, A. 2004, ApJ, 613, 159

Fukuda, H., Habe, A., \& Wada, K. 2000, ApJ, 529, 109

Furlanetto, S. R., \& Loeb, A. 2001, ApJ, 556, 619 
Gallagher, S. C., Brandt, W. N., Laor, A., Elvis, M., Mathur, S., Wills, B. J., \& Iyomoto, N. 2001, ApJ, 546, 795

Gallagher, S. C., Brandt, W. N., Sambruna, R. M., Mathur, S., \& Yamasaki, N. 1999, ApJ, 519, 549

García-Burillo, S., Combes, F., Schinnerer, E. a nd Boone, F., \& Hunt, L. K. 2005, A\&A, 441, 1011

Gebhardt, K., Bender, R., Bower, G., Dressler, A., Faber, S. M., Filippenko, A. V., Green, R., Grillmair, C., Ho, L. C., Kormendy, J., Lauer, T. R., Magorrian, J., Pinkney, J., Richstone, D., \& Tremaine, S. 2000, ApJ, 539, L13

Gnedin, N. Y. 2000, ApJ, 542, 535

Gnedin, O. Y. 2001, Classical and Quantum Gravity, 18, 3983

Gnedin, O. Y., Kravtsov, A. V., Klypin, A. A., \& Nagai, D. 2004, ApJ, 616, 16

Goldreich, P., \& Lynden-Bell, D. 1965, MNRAS, 130, 97

Governato, F., Willman, B., Mayer, L., Brooks, A., Stinson, G., Valenzuela, O., Wadsley, J., \& Quinn, T. 2007, MNRAS, 374, 1479

Haehnelt, M. G., \& Rees, M. J. 1993, MNRAS, 263, 168

Hagan, B., Ma, C.-P., \& Kravtsov, A. V. 2005, ApJ, 633, 537

Hamann, F., Korista, K. T., \& Morris, S. L. 1993, ApJ, 415, 541

Hawley, J. F., Gammie, C. F., \& Balbus, S. A. 1995, ApJ, 440, 742

Hernquist, L. 1989, Nature, 340, 687

Hewett, P. C., \& Foltz, C. B. 2003, AJ, 125, 1784

Hewett, P. C., Foltz, C. B., \& Chaffee, F. H. 1995, AJ, 109, 1498

—. 2001, AJ, 122, 518

Hoekstra, H., Mellier, Y., van Waerbeke, L., Semboloni, E., Fu, L., Hudson, M. J., Parker, L. C., Tereno, I., \& Benabed, K. 2005, First cosmic shear results from the Canada-France-Hawaii Telescope Wide Synoptic Legacy Survey

Hopkins, P. F., Cox, T. J., Kereš, D., \& Hernquist, L. 2008a, ApJS, 175, 390

Hopkins, P. F., \& Hernquist, L. 2006, Astrophys. J. Suppl., 166, 1

Hopkins, P. F., Hernquist, L., Cox, T. J., Di Matteo, T., Robertson, B., \& Springel, V. 2006, ApJS, 163, 1 
Hopkins, P. F., Hernquist, L., Cox, T. J., \& Kereš, D. 2008b, ApJS, 175, 356

Hopkins, P. F., Hernquist, L., Cox, T. J., Robertson, B., \& Krause, E. 2007, ApJ, 669,45

Hoyle, F., \& Lyttleton, R. A. 1939, in Proceedings of the Cambridge Philisophical Society, Vol. 35, Proceedings of the Cambridge Philisophical Society, 405-+

Hui, L., \& Haiman, Z. 2003, ApJ, 596, 9

Jing, Y. P., Zhang, P., Lin, W. P., Gao, L., \& Springel, V. 2006, ApJ, 640, L119

Kauffmann, G., \& Haehnelt, M. 2000, MNRAS, 311, 576

Kennicutt, Jr., R. C. 1998, ApJ, 498, 541

Kim, T.-S., Cristiani, S., \& D'Odorico, S. 2002, A\&A, 383, 747

King, A. R., \& Pringle, J. E. 2006, MNRAS, 373, L90

—. 2007, MNRAS, 377, L25

King, A. R., Pringle, J. E., \& Hofmann, J. A. 2008, MNRAS, 385, 1621

Klypin, A., Kravtsov, A. V., Bullock, J. S., \& Primack, J. R. 2001, ApJ, 554, 903

Konigl, A., \& Kartje, J. F. 1994, ApJ, 434, 446

Kormendy, J., \& Gebhardt, K. 2001, in American Institute of Physics Conference Series, Vol. 586, 20th Texas Symposium on relativistic astrophysics, ed. J. C. Wheeler \& H. Martel, 363-+

Kormendy, J., \& Kennicutt, Jr., R. C. 2004, ARA\&A, 42, 603

Kormendy, J., \& Richstone, D. 1995, ARA\&A, 33, 581

Koushiappas, S. M., Bullock, J. S., \& Dekel, A. 2004, MNRAS, 354, 292

Kravtsov, A. V. 1999, PhD thesis, AA(NEW MEXICO STATE UNIVERSITY)

-. 2003, ApJ, 590, L1

Kravtsov, A. V., Klypin, A., \& Hoffman, Y. 2002, ApJ, 571, 563

Kravtsov, A. V., Klypin, A. A., \& Khokhlov, A. M. 1997, ApJS, 111, 73

Kritsuk, A. G., Norman, M. L., Padoan, P., \& Wagner, R. 2007, ApJ, 665, 416

Krolik, J. H. 1999, Active galactic nuclei : from the central black hole to the galactic environment (Active galactic nuclei : from the central black hole to the galactic environment/Julian H. Krolik. Princeton, N. J. : Princeton University Press, c1999.) 
Krumholz, M. R., Klein, R. I., \& McKee, C. F. 2007, ApJ, 656, 959

Krumholz, M. R., McKee, C. F., \& Klein, R. I. 2006, ApJ, 638, 369

Larson, R. B. 2000, in ESA Special Publication, Vol. 445, Star Formation from the Small to the Large Scale, ed. F. Favata, A. Kaas, \& A. Wilson, 13-+

Lee, H. M. 1995, MNRAS, 272, 605

Levine, R., \& Gnedin, N. Y. 2005, ApJ, 632, 727

-. 2006, ApJ, 649, L57

Levine, R., Gnedin, N. Y., Hamilton, A. J. S., \& Kravtsov, A. V. 2007, ArXiv e-prints, 711

Li, Y., Haiman, Z., \& Mac Low, M.-M. 2007a, ApJ, 663, 61

Li, Y., Hernquist, L., Robertson, B., Cox, T. J., Hopkins, P. F., Springel, V., Gao, L., Di Matteo, T., Zentner, A. R., Jenkins, A., \& Yoshida, N. 2007b, ApJ, 665, 187

Lidz, A., Hopkins, P. F., Cox, T. J., Hernquist, L., \& Robertson, B. 2006, ApJ, 641,41

Lindt-Krieg, E., Eckart, A., Neri, R., Krips, M. a nd Pott, J.-U., García-Burillo, S., \& Combes, F. 2008, A\&A, 479, 377

Loeb, A., \& Rasio, F. A. 1994, ApJ, 432, 52

Lomb, N. R. 1976, Ap\&SS, 39, 447

Lynden-Bell, D. 1969, Nature, 223, 690

Mac Low, M.-M., \& Klessen, R. S. 2004, Reviews of Modern Physics, 76, 125

Machacek, M. E., Bryan, G. L., \& Abel, T. 2001, ApJ, 548, 509

Madau, P., Ferrara, A., \& Rees, M. J. 2001, ApJ, 555, 92

Madau, P., \& Rees, M. J. 2001, ApJ, 551, L27

Magorrian, J., Tremaine, S., Richstone, D., Bender, R., Bower, G., Dressler, A., Faber, S. M., Gebhardt, K., Green, R., Grillmair, C., Kormendy, J., \& Lauer, T. 1998, AJ, 115, 2285

Mahmood, A., Devriendt, J. E. G., \& Silk, J. 2005, MNRAS, 359, 1363

Malbon, R. K., Baugh, C. M., Frenk, C. S., \& Lacey, C. G. 2006, ArXiv Astrophysics e-prints 
Marulli, F., Crociani, D., Volonteri, M., Branchini, E., \& Moscardini, L. 2006, MNRAS, 368, 1269

Mayer, L., Kazantzidis, S., Madau, P., Colpi, M., Quinn, T., \& Wadsley, J. 2007, Science, 316, 1874

McDonald, P., \& Miralda-Escudé, J. 2001, ApJ, 549, L11

McDonald, P., Miralda-Escudé, J., Rauch, M., Sargent, W. L. W., Barlow, T. A., \& Cen, R. 2001, ApJ, 562, 52

McKee, C. F., \& Ostriker, E. C. 2007, ARA\&A, 45, 565

Menou, K., Vanden Berk, D. E., Ivezić, Ž., Kim, R. S. J., Knapp, G. R., Richards, G. T., Strateva, I., Fan, X., Gunn, J. E., Hall, P. B., Heckman, T., Krolik, J., Lupton, R. H., Schneider, D. P., York, D. G., Anderson, S. F., Bahcall, N. A., Brinkmann, J., Brunner, R., Csabai, I., Fukugita, M., Hennessy, G. S., Kunszt, P. Z., Lamb, D. Q., Munn, J. A., Nichol, R. C., \& Szokoly, G. P. 2001, ApJ, 561,645

Mestel, L. 1963, MNRAS, 126, 553

Miller, M. C., \& Ostriker, E. C. 2001, ApJ, 561, 496

Mo, H. J., \& Mao, S. 2004, MNRAS, 353, 829

Modjaz, M., Moran, J. M., Greenhill, L. J., \& Kondratko, P. T. 2005, in Astronomical Society of the Pacific Conference Series, Vol. 340, Future Directions in High Resolution Astronomy, ed. J. Romney \& M. Reid, 192-+

Mori, M., Ferrara, A., \& Madau, P. 2002, ApJ, 571, 40

Morris, S. L. 1988, ApJ, 330, L83

Nath, B. B., \& Roychowdhury, S. 2002, MNRAS, 333, 145

Navarro, J. F., Frenk, C. S., \& White, S. D. M. 1997, ApJ, 490, 493

Nayakshin, S., \& King, A. 2007, ArXiv e-prints, 705

Noguchi, M. 1988, A\&A, 203, 259

Oppenheimer, B. D., \& Davé, R. 2006, MNRAS, 373, 1265

Ossenkopf, V., \& Henning, T. 1994, A\&A, 291, 943

Ostriker, J. P., \& Peebles, P. J. E. 1973, ApJ, 186, 467

Passot, T., \& Vázquez-Semadeni, E. 1998, Phys. Rev. E, 58, 4501

Penton, S. V., Stocke, J. T., \& Shull, J. M. 2004, ApJS, 152, 29 
Polyachenko, V. L., Polyachenko, E. V., \& Strel'Nikov, A. V. 1997, Astronomy Letters, 23, 483

Porciani, C., Magliocchetti, M., \& Norberg, P. 2004, MNRAS, 355, 1010

Press, W. H., Teukolsky, S. A., Vetterling, W. T., \& Flannery, B. P. 1992, Numerical recipes in $\mathrm{C}$. The art of scientific computing (Cambridge: University Press, —c1992, 2nd ed.)

Pringle, J. E. 1981, ARA\&A, 19, 137

Quinlan, G. D., \& Shapiro, S. L. 1990, ApJ, 356, 483

Regan, M. W., \& Teuben, P. J. 2004, ApJ, 600, 595

Rice, W. K. M., Lodato, G., \& Armitage, P. J. 2005, MNRAS, 364, L56

Ricotti, M., Gnedin, N. Y., \& Shull, J. M. 2000, ApJ, 534, 41

Riffel, R. A., Storchi-Bergmann, T., Winge, C., McGregor, P. J., Beck, T., \& Schmitt, H. 2008, MNRAS, 385, 1129

Ripamonti, E., \& Abel, T. 2004, MNRAS, 348, 1019

Roberts, Jr., W. W., Huntley, J. M., \& van Albada, G. D. 1979, ApJ, 233, 67

Rudd, D. H., Zentner, A. R., \& Kravtsov, A. V. 2008, ApJ, 672, 19

Ruszkowski, M., Brüggen, M., \& Begelman, M. C. 2004, ApJ, 615, 675

Salpeter, E. E. 1964, ApJ, 140, 796

Scannapieco, E., Ferrara, A., \& Madau, P. 2002, ApJ, 574, 590

Scannapieco, E., \& Oh, S. P. 2004, ApJ, 608, 62

Scannapieco, E., Silk, J., \& Bouwens, R. 2005, ApJ, 635, L13

Schaye, J., Theuns, T., Rauch, M., Efstathiou, G., \& Sargent, W. L. W. 2000, MNRAS, 318, 817

Schirber, M., \& Bullock, J. S. 2003, ApJ, 584, 110

Schneider, R., Ferrara, A., Ciardi, B., Ferrari, V., \& Matarrese, S. 2000, MNRAS, 317,385

Shapiro, P. R., Iliev, I. T., \& Raga, A. C. 2004, MNRAS, 348, 753

Shlosman, I., \& Begelman, M. C. 1989, ApJ, 341, 685

Shlosman, I., Begelman, M. C., \& Frank, J. 1990, Nature, 345, 679 
Shlosman, I., Frank, J., \& Begelman, M. C. 1989, Nature, 338, 45

Sijacki, D., Springel, V., Di Matteo, T., \& Hernquist, L. 2007, MNRAS, 380, 877

Silk, J., \& Rees, M. J. 1998, A\&A, 331, L1

Simkin, S. M., Su, H. J., \& Schwarz, M. P. 1980, ApJ, 237, 404

Soltan, A. 1982, MNRAS, 200, 115

Somerville, R. S. 2002, ApJ, 572, L23

Spaans, M., \& Silk, J. 2006, ApJ, 652, 902

Springel, V. 2005, MNRAS, 364, 1105

Springel, V., Di Matteo, T., \& Hernquist, L. 2005, MNRAS, 361, 776

Springel, V., \& Hernquist, L. 2003, MNRAS, 339, 289

Stinson, G., Seth, A., Katz, N., Wadsley, J., Governato, F., \& Quinn, T. 2006, MNRAS, 373, 1074

Stocke, J. T., Morris, S. L., Weymann, R. J., \& Foltz, C. B. 1992, ApJ, 396, 487

Storchi-Bergmann, T., Dors, Jr., O. L., Riffel, R. A. a nd Fathi, K., Axon, D. J., Robinson, A., Marconi, A., \& Östlin, G. 2007, ApJ, 670, 959

Susa, H., \& Umemura, M. 2004, ApJ, 600, 1

Tassis, K., Abel, T., Bryan, G. L., \& Norman, M. L. 2003, ApJ, 587, 13

Tassis, K., Kravtsov, A. V., \& Gnedin, N. Y. 2008, ApJ, 672, 888

Tegmark, M., Silk, J., \& Evrard, A. 1993, ApJ, 417, 54

Theuns, T., Zaroubi, S., Kim, T.-S., Tzanavaris, P., \& Carswell, R. F. 2002, MNRAS, 332, 367

Toomre, A. 1964, ApJ, 139, 1217

Tremaine, S., Gebhardt, K., Bender, R., Bower, G., Dressler, A., Faber, S. M., Filippenko, A. V., Green, R., Grillmair, C., Ho, L. C., Kormendy, J., Lauer, T. R., Magorrian, J., Pinkney, J., \& Richstone, D. 2002, ApJ, 574, 740

Truelove, J. K., Klein, R. I., McKee, C. F., Holliman, II, J. H., Howell, L. H., \& Greenough, J. A. 1997, ApJ, 489, L179+

Truelove, J. K., Klein, R. I., McKee, C. F., Holliman, II, J. H., Howell, L. H., Greenough, J. A., \& Woods, D. T. 1998, ApJ, 495, 821 
Ulrich, M.-H. 1988, MNRAS, 230, 121

Umemura, M., Loeb, A., \& Turner, E. L. 1993, ApJ, 419, 459

Valageas, P., \& Silk, J. 1999, A\&A, 350, 725

Vazquez-Semadeni, E. 1994, ApJ, 423, 681

Vittorini, V., Shankar, F., \& Cavaliere, A. 2005, MNRAS, 363, 1376

Vlemmings, W. H. T., Bignall, H. E., \& Diamond, P. J. 2007, ApJ, 656, 198

Volonteri, M., \& Rees, M. J. 2005, ApJ, 633, 624

-. 2006, ApJ, 650, 669

Wada, K. 2001, ApJ, 559, L41

Wada, K. 2004, in Coevolution of Black Holes and Galaxies, ed. L. C. Ho, 186-+

Wada, K., Meurer, G., \& Norman, C. A. 2002, ApJ, 577, 197

Wada, K., \& Norman, C. A. 2001, ApJ, 547, 172

-. 2007, ApJ, 660, 276

Wada, K., \& Venkatesan, A. 2003, ApJ, 591, 38

Weymann, R. 1997, in Astronomical Society of the Pacific Conference Series, Vol. 128, Mass Ejection from Active Galactic Nuclei, ed. N. Arav, I. Shlosman, \& R. J. Weymann, 3-+

Weymann, R. J., Morris, S. L., Foltz, C. B., \& Hewett, P. C. 1991, ApJ, 373, 23

White, M. 2004, Astroparticle Physics, 22, 211

Willott, C. J., Rawlings, S., Blundell, K. M., \& Lacy, M. 1999, MNRAS, 309, 1017

Wise, J. H. 2007, PhD thesis, Stanford University

Wise, J. H., Turk, M. J., \& Abel, T. 2007, ArXiv e-prints, 710

Wong, T., \& Blitz, L. 2002, ApJ, 569, 157

Wyithe, J. S. B., \& Loeb, A. 2003, ApJ, 595, 614

Wyse, R. F. G. 2004, ApJ, 612, L17

Yoo, J., \& Miralda-Escudé, J. 2004, ApJ, 614, L25 
Young, J. S., Allen, L., Kenney, J. D. P., Lesser, A., \& Rownd, B. 1996, AJ, 112, 1903

Yu, Q., \& Tremaine, S. 2002, MNRAS, 335, 965

Zhan, H., \& Knox, L. 2004, ApJ, 616, L75

Zweibel, E. G., \& Heiles, C. 1997, Nature, 385, 131 